\title{
Natural SUSY at LHC with right-sneutrino LSP
}

\author{
Arindam Chatterjee, ${ }^{a}$ Juhi Dutta ${ }^{b}$ and Santosh Kumar Rai ${ }^{b}$ \\ ${ }^{a}$ Indian Statistical Institute, \\ 203 B.T. Road, Kolkata-700108, India \\ ${ }^{b}$ Regional Centre for Accelerator-based Particle Physics, \\ Harish-Chandra Research Institute, HBNI, \\ Chhatnag Road, Jhusi, Allahabad-211019, India \\ E-mail: arindam.chatterjee@gmail.com, juhidutta@hri.res.in, \\ skrai@hri.res.in
}

ABSTRACT: We study an extension of the minimal supersymmetric standard model (MSSM) with additional right-handed singlet neutrino superfields. While such an extension incorporates a mechanism for the neutrino mass, it also opens up the possibility of having the right-sneutrinos $(\widetilde{\nu})$ as the lightest supersymmetric particle (LSP). In this work, we focus on the viability of rather small $(\lesssim 500 \mathrm{GeV})$ higgsino mass parameter $(\mu)$, an important ingredient for "naturalness", in the presence of such a LSP. For simplicity, we assume that the bino and wino mass parameters are much heavier; thus we only consider (almost) pure and compressed higgsino-like states, with small $\mathcal{O}\left(10^{-2}\right)$ gaugino admixture which nevertheless still affect the decay of the low-lying higgsino-like states, thus significantly affecting the proposed signatures at colliders. Considering only prompt decays of the higgino-like states, especially the lightest chargino, we discuss the importance of leptonic channels consisting of up to two leptons with large missing transverse energy to probe this scenario at the Large Hadron Collider (LHC). In addition we also comment on the dark matter predictions for the studied scenario.

Keywords: Supersymmetry Phenomenology

ARXIV EPRINT: 1710.10617 


\section{Contents}

1 Introduction 1

2 The model 3

2.1 The (s)neutrino sector 4

$\begin{array}{lll}2.2 & \text { The electroweakino sector } & 6\end{array}$

2.3 Compressed Higgsino spectrum and its decay properties 9

3 Survey of the relevant parameter space $\quad 15$

$\begin{array}{lll}3.1 \text { General constraints } & 15\end{array}$

$\begin{array}{lll}3.1 .1 & \text { Implication from neutrino mass } & 15\end{array}$

$\begin{array}{ll}3.1 .2 & \text { Implications for dark matter } \\ \end{array}$

4 Signatures at LHC $\quad 17$

4.1 Constraints on electroweakino sector from LHC 19

4.2 Impact of additional related searches at LHC 20

4.3 Benchmarks 22

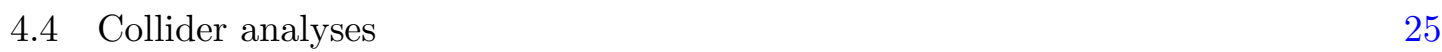

4.5 Mono-lepton $+\leq 1$ jet $+\mathbb{E}_{T}$ signal 26

4.6 Di-lepton +0 jet $+\mathbb{E}_{T}$ signal 30

4.6.1 Opposite sign di-lepton +0 jet $+\mathbb{E}_{T}$ signal 30

4.6.2 Same sign di-leptons +0 jet $+\mathbb{E}_{T}$ signal 34

5 Conclusion $\quad 38$

\section{Introduction}

The TeV scale limits from LHC searches on the masses of strongly interacting supersymmetric particles set a dismal tone for naturalness concerns, a prime motivation for invoking Supersymmetry (SUSY) in particle physics studies. While several studies in the literature attempt to quantify "naturalness" in a supersymmetric scenario, the interpretation and the measure of naturalness are often debated [1-6]. Nevertheless, in minimal supersymmetric extensions of the standard model (MSSM), a small value of the higgsino mass parameter $\mu$ and possibly with light [1-4] or a rather light stop squarks and gluinos $(\lesssim 1.5 \mathrm{TeV})[5-8]$ remain desirable in "natural" scenarios at the electro-weak (EW) scale. However, even with not-so-light strong sector $[9,10]$, "natural" scenarios without much fine-tuning is possible impressing the fact that low $|\mu|$ is of more essence to the "natural" scenarios at EW scale.

While the constraints on stop squarks and gluinos are rather stringent due to their large production cross-section at the LHC, the weakly interacting sector with rather light 
electroweakinos in general, and higgsinos in particular, remain viable [11, 12]. There have been several analyses on light electroweakinos, assuming a simplified spectra with one or more specific decay channels [13-26]. Further, the constraints on the mass of the light higgsino-like states have been studied in detail because of their importance in a "natural" supersymmetric scenario [19, 27-33]. However, note that these analyses assume the lightest neutralino as the lightest supersymmetric particle (LSP). In scenarios with conserved Rparity, the search strategies, and therefore the limits of various sparticle masses, depend on the nature of the LSP. This is because in such scenarios the LSP appears at the end of the decay chain of each sparticle, therefore dictating the possible search channels. This warrants investigation of supersymmetric scenarios with different types of LSP. While within the paradigm of the MSSM, the lightest neutralino is the LSP, and most supersymmetric searches are based on the same assumption. There have been studies with gravitino LSP, discussing implications on cosmology and signatures at the LHC [34-53]. In other simple extensions, axion and/or axino as the LSP [54-58] and right-sneutrino LSP have also been considered in minimal extensions of the MSSM [59-70]. While the former sets out to resolve the strong $\mathrm{CP}$-problem, the latter provides a weak-scale solution to the neutrino mass generation issue, an important aspect missing in the MSSM.

In this work we consider a similar extension to the MSSM with three generations of right-neutrino superfields. This scenario, which provides a weak-scale solution to the neutrino mass generation issue, has been widely studied in supersymmetric extensions. While the left-sneutrinos have been ruled out as a Dark Matter (DM) candidate long ago, thanks to the stringent limit from direct detection experiments [71], right-sneutrinos continue to be widely studied as a candidate for DM in simple extensions of the MSSM [59$63,65-68,72]$. In its simplest incarnation as ours, the right-sneutrinos at EW scale remain very weakly interacting, thanks to the small Yukawa coupling $\mathcal{O}\left(10^{-6}-10^{-7}\right)$ determining their coupling strength to other particles. However, as in the case of charged sfermions, a rather large value of the corresponding tri-linear soft supersymmtry breaking parameter can induce significant left-admixture in a dominantly right-sneutrino and therefore can substantially increase the interaction strengths $[66,67,72]$. In both of these scenarios, DM aspects as well as search strategies at LHC have been studied for certain choices of the SUSY spectra [64, 73-79].

We note that in the light of "naturalness", it becomes equally important to investigate the supersymmetric spectrum in such a scenario. In particular we focus on a minimalistic spectrum, motivated by "naturalness" at the EW scale, with light higgsino-like states and a right-sneutrino LSP. However, analysing collider signatures from the third generation squarks and gluinos will be beyond the scope of the present work and will be addressed in a subsequent extension. For the present case, the strongly interacting sparticles have been assumed to be very heavy adhering to the "naturalness" scheme proposed in refs. [9, 10]. Further, we will also assume the gaugino mass parameters to be large enough $(\gtrsim \mathcal{O}(1) \mathrm{TeV})$. Thus the light electroweakinos are higgsino-dominated states. Note that the presence of a mixed right-sneutrino as the LSP can lead to a very different signature from the compressed higgsino-like states, mostly due to the leptonic decay of the light chargino. Although leptonic channels provide a cleaner environment for new physics searches at a 
hadron machine such as the LHC, one expects that the level of compression in the mass spectra of the electroweakinos would also play a major role in determining the efficacy of the leptonic channels. We investigate the prospects of discovery of such channels at the $13 \mathrm{TeV}$ run of LHC. We focus on the following apsects in our study:

- We consider a right-sneutrino LSP along with a compressed electroweakino sector sitting above the LSP, where the lighter states are almost Higgsino-like with a very small admixture of gauginos.

- We give a detailed account of how the decay of the light electroweakinos depend on the various supersymmetric parameters that govern the mixing, mass splitting and, in which region of the parameter space the decays are prompt. We also highlight how even the smallest gaugino admixture plays a significant role in their decays.

- We comment on the DM predictions for a thermal as well as non-thermal nature of the right-sneutrino DM candidate in regions of parameter space of our interest.

- We then look at possible leptonic signals that arise from such a spectrum and analyze the signal at LHC.

The article is organized as follows. In section 2 we discuss the model and the underlying particle spectrum of interest in detail. In the following section 3 we focus on identifying the parameter space satisfying relevant constraints as well as implications on neutrino sector and a sneutrino as DM. In section 4 we discuss the possible signatures at LHC and present our analysis for a few representative points in the model parameter space. We finally conclude in section 5 .

\section{The model}

We consider an extension to the Minimal Supersymmetric Standard Model (MSSM) by introducing a right-chiral neutrino superfield for each generation. This extension addresses the important issue of neutrino mass generation which is otherwise absent in the MSSM. In particular, we adopt a phenomenological approach for "TeV type-I seesaw mechanism". The superpotential, suppressing the generation indices, is given by $[59,72,80]$ :

$$
\mathcal{W} \supset \mathcal{W}_{\mathrm{MSSM}}+y_{\nu} \hat{L} \hat{H}_{u} \hat{N}^{c}+\frac{1}{2} M_{R} \hat{N}^{c} \hat{N}^{c}
$$

where $y_{\nu}$ is the neutrino Yukawa coupling, $\hat{L}$ is the left-chiral lepton doublet superfield, $\hat{H}_{u}$ is the Higgs up-type chiral superfield and $\hat{N}$ is the right-chiral neutrino superfield. Besides the usual MSSM superpotential terms denoted by $\mathcal{W}_{\text {MSSM }}$, we now have an added Yukawa interaction term involving the left-chiral superfield $\hat{L}$ coupled to the up-type Higgs superfield $\hat{H}_{u}$, and $\hat{N}$. SM neutrinos obtain a Dirac mass $m_{D}$ after electroweak symmetry breaking once the neutral Higgs field obtains a vacuum expectation value (vev) $v_{u}$, such that $m_{D}=y_{\nu} v_{u}$. The third term $\frac{1}{2} M_{R} \hat{N}^{c} \hat{N}^{c}$ is a lepton-number violating $(\not L)$ term $(\triangle L=2)$. 
In addition to the MSSM contributions, the soft-supersymmetry breaking scalar potential receives additional contributions as follows:

$$
\mathcal{V}^{\text {soft }} \supset \mathcal{V}_{\text {MSSM }}^{\text {soft }}+m_{R}^{2}|\widetilde{N}|^{2}+\frac{1}{2} B_{M} \widetilde{N}^{c} \widetilde{N}^{c}+\left(T_{\nu} \widetilde{L} \cdot H_{u} \widetilde{N}^{c}+\text { h.c. }\right)
$$

where $m_{R}^{2}$ is the soft-supersymmetry breaking mass parameter for the sneutrino, $B_{M}$ is the soft mass-squared parameter corresponding to the lepton-number violating term and $T_{\nu}$ is the soft-supersymmetry breaking L-R mixing term in the sneutrino sector. We have suppressed the generation indices both for the superpotential as well as for the soft supersymmetry-breaking terms so far.

Note that a small $\mu$-parameter is critical to ensure the absence of any fine-tuning at the EW scale $\left(\Delta_{\mathrm{EW}}\right)$ [5-8]. Fine-tuning arises if there is any large cancellation involved at the EW scale in the right hand side of the following relation $[1,2]$ :

$$
\frac{M_{Z}^{2}}{2}=\frac{m_{H_{d}}^{2}+\Sigma_{d}-\left(m_{H_{u}}^{2}+\Sigma_{u}\right) \tan \beta^{2}}{\tan \beta^{2}-1}-\mu^{2},
$$

where $m_{H_{u}}^{2}, m_{H_{d}}^{2}$ denote the soft-supersymmetry breaking terms for the up-type and the down-type Higgses at the supersymmetry breaking mass scale (which is assumed to be the geometric mean of the stop masses in the present context) and $\tan \beta$ denotes the ratio of the respective vevs while $\Sigma_{u}$ and $\Sigma_{d}$ denote the radiative corrections. Note that, since we are not considering any specific high-scale framework in the present context, we are only concerned about the EW fine-tuning. Typically $\Delta_{\text {EW }} \lesssim 30$ is achieved with $|\mu| \lesssim 300 \mathrm{GeV}[5-8]$. The assurance of EW naturalness is the prime motivation in exploring small $\mu$ scenarios. However it is quite possible that obtaining such a spectrum from a high-scale theory may require larger fine-tuning among the high-scale parameters and the corresponding running involved, especially considering that $m_{H_{u}}$ evolves significantly to ensure radiative EW symmetry breaking. Therefore, $\Delta_{\mathrm{EW}}$ can be interpreted as a lower bound on fine-tuning measure [5-8]. Note that, stop squarks and gluinos contribute to the radiative corrections to $m_{H_{u}}$ at one and two-loop levels respectively. It has been argued [9, 10] that an EW fine-tuning of less than about 30 can be achieved with $\mu \lesssim 300 \mathrm{GeV}$ and with stop squarks and (gluinos) as heavy as about $3 \mathrm{TeV}(4 \mathrm{TeV})$. It is, therefore, important to probe possible scenarios with low $\Delta_{\mathrm{EW}}$ and therefore with low $|\mu|$.

\subsection{The (s)neutrino sector}

In presence of the soft-supersymmetry-breaking terms $B_{M}$, a split is generated between the CP-even and the CP-odd part of right-type sneutrino fields. In terms of CP eigenstates we can write: $\widetilde{\nu}_{L}=\frac{\widetilde{\nu}_{L}^{e}+i \widetilde{\nu}_{L}^{o}}{\sqrt{2}} ; \widetilde{\nu}_{R}=\frac{\widetilde{\nu}_{R}^{e}+i \widetilde{\nu}_{R}^{o}}{\sqrt{2}}$, where superscripts $e$, o denote "even" and "odd" respectively. The sneutrino $(\widetilde{\nu})$ mass-squared matrices in the basis $\widetilde{\nu}^{e}=\left\{\widetilde{\nu}_{L}^{e}, \widetilde{\nu}_{R}^{e}\right\}^{T}$ and $\widetilde{\nu}^{o}=\left\{\widetilde{\nu}_{L}^{o}, \widetilde{\nu}_{R}^{o}\right\}^{T}$ are given by,

$$
\mathcal{M}^{j 2}=\left(\begin{array}{cc}
m_{L L}^{2} & m_{L R}^{j 2} \\
m_{L R}^{j 2} & m_{R R}^{j 2}
\end{array}\right),
$$


where,

$$
\begin{aligned}
& m_{L L}^{2}=m_{L}^{2}+\frac{1}{2} m_{Z}^{2} \cos 2 \beta+m_{D}^{2}, \\
& m_{L R}^{j 2}=\left(T_{\nu} \pm y_{\nu} M_{R}\right) v \sin \beta-\mu m_{D} \cot \beta \\
& m_{R R}^{j 2}=m_{R}^{2}+m_{D}^{2}+M_{R}^{2} \pm B_{M}
\end{aligned}
$$

with $j \in\{e, o\}$ and the ' + ' and the '-' signs correspond to $j=e$ and $j=o$ respectively, and $v=\sqrt{v_{u}^{2}+v_{d}^{2}}=174 \mathrm{GeV}$, where $v_{u}, v_{d}$ denotes the vevs of the up-type and the down-type CP-even neutral Higgs bosons. Further, we have assumed $T_{\nu}$ to be real and with no additional CP-violating parameters in the sneutrino sector. The physical masses and the mass eigenstates can be obtained by diagonalizing these matrices. The eigenvalues are given by:

$$
m_{1,2}^{j 2}=\frac{1}{2}\left(m_{L L}^{2}+m_{R R}^{j 2} \pm \sqrt{\left(m_{L L}^{2}-m_{R R}^{j 2}\right)^{2}+4 m_{L R}^{j 4}}\right) .
$$

The corresponding mass eigenstates are give by,

$$
\begin{aligned}
& \widetilde{\nu}_{1}^{j}=\cos \varphi^{j} \widetilde{\nu}_{L}^{j}-\sin \varphi^{j} \widetilde{\nu}_{R}^{j} \\
& \widetilde{\nu}_{2}^{j}=\sin \varphi^{j} \widetilde{\nu}_{L}^{j}+\cos \varphi^{j} \widetilde{\nu}_{R}^{j} .
\end{aligned}
$$

The mixing angle $\theta=\frac{\pi}{2}-\varphi$ is given by,

$$
\sin 2 \theta^{j}=\frac{\left(T_{\nu} \pm y_{\nu} M_{R}\right) v \sin \beta-\mu m_{D} \cot \beta}{m_{2}^{j 2}-m_{1}^{j 2}},
$$

where $j$ denotes $\mathrm{CP}$-even $(e)$ or $\mathrm{CP}$-odd $(o)$ states.

The off-diagonal term involving $T_{\nu}$ is typically proportional to the coupling $y_{\nu}$, ensuring that the left-right (L-R) mixing is small. However, the above assumption relies on the mechanism of supersymmetry-breaking and may be relaxed. The phenomenological choice of a large $T_{\nu} \sim \mathcal{O}(1) \mathrm{GeV}$ leads to increased mixing between the left and right components of the sneutrino flavor eigenstates in the sneutrino mass eigenstates [66, 67, 72]. Further, if the denominator in eq. (2.6) is suitably small, it can also lead to enhanced mixing.

As for the neutrinos, at tree-level with $M_{R} \gg 1 \mathrm{eV}$, their masses are given by $m_{\nu} \simeq \frac{y_{\nu}^{2} v_{u}^{2}}{M_{R}}$, as in the case of Type-I see-saw mechanism [81-83]. Thus, with $M_{R} \sim \mathcal{O}(100) \mathrm{GeV}$, neutrino masses of $\mathcal{O}(0.1) \mathrm{eV}$ requires $y_{\nu} \sim 10^{-6}-10^{-7}$. Although we have ignored the flavor indices in the above discussion of the sneutrino sector, the neutrino oscillation experiments indicate that these will play an important role in the neutrino sector. We will assume that the leptonic Yukawa couplings are flavor diagonal, and that the only source of flavor mixing arises from $y_{\nu}$ [84]; see also [85, 86]. Further, at one-loop, flavor diagonal $B_{M}$ can also contribute to the neutrino mass matrix $[80,87]$ which can be quite significant in the presence of large $T_{\nu}$ in particular. ${ }^{1}$ The dominant contribution to the Majorana mass of the active neutrino arises from the sneutrino-gaugino loop as shown in figure 1. The

\footnotetext{
${ }^{1}$ Note that flavor off-diagonal terms in $B_{M}$ can lead to flavor mixing in the neutrino sector via higher order effects which we avoid in our discussions for simplicity.
} 


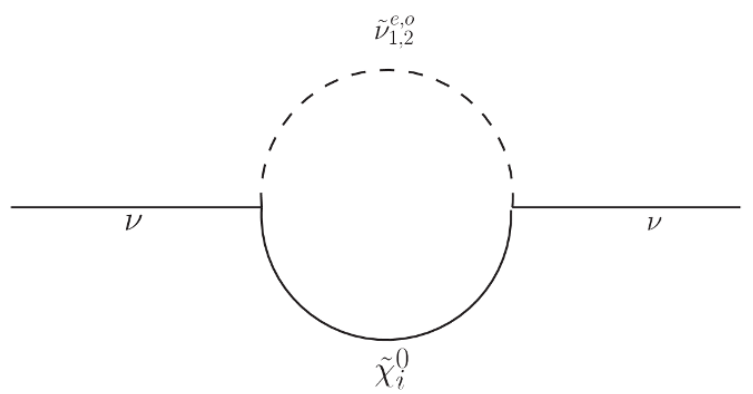

Figure 1. Schematic diagram showing the leading one-loop contribution to the light neutrino mass.

contributions from the loop are proportional to the mass splitting between the CP-even and the CP-odd left-sneutrino state which makes it significant in the presence of a rather large $T_{\nu}$ which is responsible for left-right mixing in the sneutrino sector (see eq. (2.6)). These additional contributions to the neutrino mass give significant constraints in the $\left\{T_{\nu}, B_{M}\right\}$ parameter space.

Finally, some comments on the scenario with $M_{R}=0$ and $B_{M}=0$ are in order. With $M_{R}=0$ (and $B_{M}=0$ ), only Dirac mass terms would be present for neutrinos, which is given by $y_{\nu} v_{u}$. The oscillation data for neutrinos can only be satisfied by assuming $y_{\nu}$ (and/or $T_{\nu}$, at one-loop order) to be flavor off-diagonal. In addition, $\mathcal{O}(0.1) \mathrm{eV}$ neutrino mass, then, requires a very small $y_{\nu} \simeq 10^{-11}$.

In the sneutrino sector, the relevant mass eigenstates may be obtained simply by substituting $M_{R}=0=B_{M}$ in equations (2.2), (2.3), (2.4). Since the mass matrices for both $\mathrm{CP}$-even and the $\mathrm{CP}$-odd sneutrinos are identical in this scenario, any splitting between the corresponding mass eigenstates would be absent. Consequently there will be only two complex-scalar mass eigenstates $\tilde{\nu}_{1}, \tilde{\nu}_{2}$. Also, there will be no large one-loop contribution to the Majorana neutrino mass, relaxing the constraint on large $T_{\nu}$ significantly.

\subsection{The electroweakino sector}

The other relevant sector for our study is the chargino-neutralino sector, in particular the higgsino-like states. This sector resembles the chargino-neutralino sector of the MSSM. The tree-level mass term for the charginos, in the gauge eigen-basis, can be written as [88]

$$
-\mathcal{L}_{\text {mass }}^{\mathrm{c}}=\psi^{-T} M^{c} \psi^{+}+\text {h.c. }
$$

where,

$$
\psi^{+}=\left(\widetilde{W}^{+}, \quad \tilde{h}_{2}^{+}\right)^{T}, \quad \psi^{-}=\left(\widetilde{W}^{-}, \tilde{h}_{1}^{-}\right)^{T}
$$

are column vectors whose components are Weyl spinors. The mass matrix $M^{\mathrm{c}}$ is given by

$$
M^{\mathrm{c}}=\left(\begin{array}{cc}
M_{2} & \sqrt{2} M_{W} \sin \beta \\
\sqrt{2} M_{W} \cos \beta & \mu
\end{array}\right) .
$$

In the above equation, $M_{2}$ is the supersymmetry breaking $\mathrm{SU}(2)$ gaugino (wino) mass parameter, $\mu$ is the supersymmetric higgsino mass parameter, $M_{W}$ is the mass of the $W$ 
boson, and $\tan \beta$ is the ratio of vevs as described before. The non-symmetric $M^{\mathrm{c}}$ can be diagonalized with a bi-unitary transformation using the unitary matrices $U$ and $V$ to obtain the diagonal mass matrix,

$$
M_{D}^{\mathrm{c}}=U^{*} M^{\mathrm{c}} V^{-1}=\operatorname{Diagonal}\left(m_{\tilde{\chi}_{1}^{+}} m_{\tilde{\chi}_{2}^{+}}\right) .
$$

The eigenstates are ordered in mass such that $m_{\tilde{\chi}_{1}^{+}} \leq m_{\tilde{\chi}_{2}^{+}}$. The left- and right-handed components of the corresponding Dirac mass eigenstates, the charginos $\tilde{\chi}_{i}^{+}$with $i \in\{1,2\}$, are

$$
P_{L} \tilde{\chi}_{i}^{+}=V_{i j} \psi_{j}^{+}, \quad P_{R} \tilde{\chi}_{i}^{+}=U_{i j}^{*} \overline{\psi_{j}^{-}},
$$

where $P_{L}$ and $P_{R}$ are the usual projectors, $\overline{\psi_{j}^{-}}=\psi_{j}^{-\dagger}$, and summation over $j$ is implied.

For the electrically neutral neutralino states, in the gauge eigenbasis, $\psi^{0}=\left(\tilde{B^{0}}, \widetilde{W}^{3}, \tilde{h}_{1}^{0}, \tilde{h}_{2}^{0}\right)^{T}$, the tree level mass term is given by [88]

$$
-\mathcal{L}_{\text {mass }}^{\mathrm{n}}=\frac{1}{2} \psi^{0 T} M^{n} \psi^{0}+\text { h.c. }
$$

The neutralino mass matrix $M^{\mathrm{n}}$ can be written as

$$
M^{\mathrm{n}}=\left(\begin{array}{cccc}
M_{1} & 0 & -M_{Z} s_{W} c_{\beta} & M_{Z} s_{W} s_{\beta} \\
0 & M_{2} & M_{Z} c_{W} c_{\beta} & -M_{Z} c_{W} s_{\beta} \\
-M_{Z} s_{W} c_{\beta} & M_{Z} c_{W} c_{\beta} & 0 & -\mu \\
M_{Z} s_{W} s_{\beta} & -M_{Z} c_{W} s_{\beta} & -\mu & 0
\end{array}\right) .
$$

In the above mass matrix $s_{W}, s_{\beta}, c_{W}$ and $c_{\beta}$ stand for $\sin \theta_{W}, \sin \beta, \cos \theta_{W}$ and $\cos \beta$ respectively while $\theta_{W}$ is the weak mixing angle. $M_{Z}$ is the mass of the $Z$ boson, and $M_{1}$ is the supersymmetry breaking $\mathrm{U}(1)_{Y}$ gaugino (bino) mass parameter. $M^{\mathrm{n}}$ can be diagonalized by a unitary matrix $N$ to obtain the masses of the neutralinos as follows,

$$
M_{D}^{\mathrm{n}}=N^{*} M^{\mathrm{n}} N^{-1}=\operatorname{Diagonal}\left(m_{\tilde{\chi}_{1}^{0}} m_{\tilde{\chi}_{2}^{0}} m_{\tilde{\chi}_{3}^{0}} m_{\tilde{\chi}_{4}^{0}}\right)
$$

Again, without loss of generality, we order the eigenvalues such that $m_{\tilde{\chi}_{1}^{0}} \leq m_{\tilde{\chi}_{2}^{0}} \leq$ $m_{\tilde{\chi}_{3}^{0}} \leq m_{\tilde{\chi}_{4}^{0}}$.

The left-handed components of the corresponding mass eigenstates, described by fourcomponent Majorana neutralinos $\tilde{\chi}_{i}^{0}$ with $i \in\{1,2,3,4\}$, may be obtained as,

$$
P_{L} \tilde{\chi}_{i}^{0}=N_{i j} \psi_{j}^{0}
$$

where summation over $j$ is again implied; the right-handed components of the neutralinos are determined by the Majorana condition $\tilde{\chi}_{i}^{c}=\tilde{\chi}_{i}$, where the superscript $c$ stands for charge conjugation.

Since the gaugino mass parameters do not affect "naturalness", for simplicity we have assumed $M_{1}, M_{2} \gg|\mu|$. In this simple scenario there are only three low-lying higgsinolike states, $\tilde{\chi}_{1}^{0}, \tilde{\chi}_{2}^{0}$ and $\tilde{\chi}_{1}^{ \pm}$. The EW symmetry breaking induces mixing between the gaugino and the higgsino-like states, via the terms proportional to $M_{Z}, M_{W}$ in the mass matrices above. The contributions of the right-chiral neutrino superfields to the chargino 
and neutralino mass matrices are negligible, thanks to the smallness of $y_{\nu}\left(\simeq 10^{-6}\right)$. Thus lightest neutralino and charginos are expected to be nearly the same as in the MSSM. Following [89] (see also [90]), in the limit $M_{1}, M_{2} \gg|\mu|$, we give the analytical expression for the masses below,

$$
\begin{aligned}
m_{\tilde{\chi}_{1}^{ \pm}} & =|\mu|\left(1-\frac{M_{W}^{2} \sin 2 \beta}{\mu M_{2}}\right)+\mathcal{O}\left(M_{2}^{-2}\right)+\text { rad.corr. } \\
m_{\tilde{\chi}_{a, s}^{0}} & = \pm \mu-\frac{M_{Z}^{2}}{2}(1 \pm \sin 2 \beta)\left(\frac{\sin \theta_{W}^{2}}{M_{1}}+\frac{\cos \theta_{W}^{2}}{M_{2}}\right)+\text { rad.corr. }
\end{aligned}
$$

where the subscripts $s(a)$ denote symmetric (anti-symmetric) states respectively, and the sign of the eigenvalues have been retained. For the symmetric state $N_{i 3}, N_{i 4}$ share the same sign, while for the anti-symmetric state there is a relative sign between these two terms. Although the leading contribution to the mass eigenvalues are given by $|\mu|$ (which receives different radiative corrections in $M^{n}$ and $M^{c}$ ), $M_{1}, M_{2}$ and $\tan \beta$ affects the mass splitting between the three light higgsino-like states due to non-negligible gaugino-higgsino mixing. The radiative corrections, mostly from the third generation (s)quarks, contribute differently for $m_{\tilde{\chi}_{1}^{ \pm}}$and $m_{\tilde{\chi}_{1,2}^{0}}$ and have been estimated in [89, 91-93]. As we are interested in a spectrum where the lighter chargino and the neutralinos play a major role and the knowledge of their mass differences would become crucial, it is necessary to explore what role the relevant SUSY parameters have in contributing to the masses of the higgsino dominated states. It is quite evident from our choice of large $M_{1}$ and $M_{2}$ that the three states according to eq. (2.16) would be closely spaced.

We now look at how the variation of the above gaugino parameters affect the shift in mass of $m_{\tilde{\chi}_{1}^{ \pm}}$and $m_{\tilde{\chi}_{1,2}^{0}}$. Assuming $\mu=300 \mathrm{GeV}, \tan \beta=5$, in figure 2 we show the variation of the mass differences $\Delta m_{1}=m_{\widetilde{\chi}_{1}^{ \pm}}-m_{\widetilde{\chi}_{1}^{0}}$ and $\Delta m_{2}=m_{\widetilde{\chi}_{2}^{0}}-m_{\widetilde{\chi}_{1}^{ \pm}}$as a function of the gaugino mass parameters. $M_{1}$ and $M_{2}$ have been varied from $500 \mathrm{GeV}$ to $3 \mathrm{TeV}$. Further, we have set $T_{t}=2.9 \mathrm{TeV}, M_{Q_{3}}=1.3 \mathrm{TeV}, M_{U_{3}}=2 \mathrm{TeV}$ and $M_{3}=2 \mathrm{TeV}$. We have used SARAH $[94,95]$ to generate model files for SPheno [96, 97], and have used the same to estimate the masses. Since SLHA [98] convention has been followed, the input parameters, as shown in the figures above, are interpreted as $\overline{\mathrm{DR}}$ parameters at $\sim 1.6 \mathrm{TeV}$. Note that the same model and spectrum generators have been used for all subsequent figures. The following features are noteworthy from figure $2:^{2}$

- For $\mu>0 ; M_{1}, M_{2} \gg \mu$ : here $\tilde{\chi}_{2}^{0}$ is the heaviest higgsino-like state while $\tilde{\chi}_{1}^{ \pm}$remains between the two neutralinos. For a fixed $M_{1} \gg|\mu|$, the mass difference $\Delta m_{1}$ increases as $M_{2}$ decreases. This feature can be simply understood from eq. (2.16). A similar conclusion also holds for $\Delta m_{2}$. Further, as shown in panels (a) and (b) of figure 2, the variation in $\Delta m_{2}$ is larger compared to $\Delta m_{1}$ in this case.

- For $\mu>0 ; M_{1}<0$ : we find that negative $M_{1}$ can lead to negative $\Delta m_{1}$, since the lightest chargino can become lighter than this state for a wide range of $M_{2}[29,30,38]$.

\footnotetext{
${ }^{2}$ Although our numerical analysis, as shown in figure 2, includes radiative corrections, the generic features also appear at the tree-level for $|\mu|=300 \mathrm{GeV}, M_{1}, M_{2} \gg|\mu|$ and $\tan \beta=5$. We have checked this using a Mathematica code.
} 

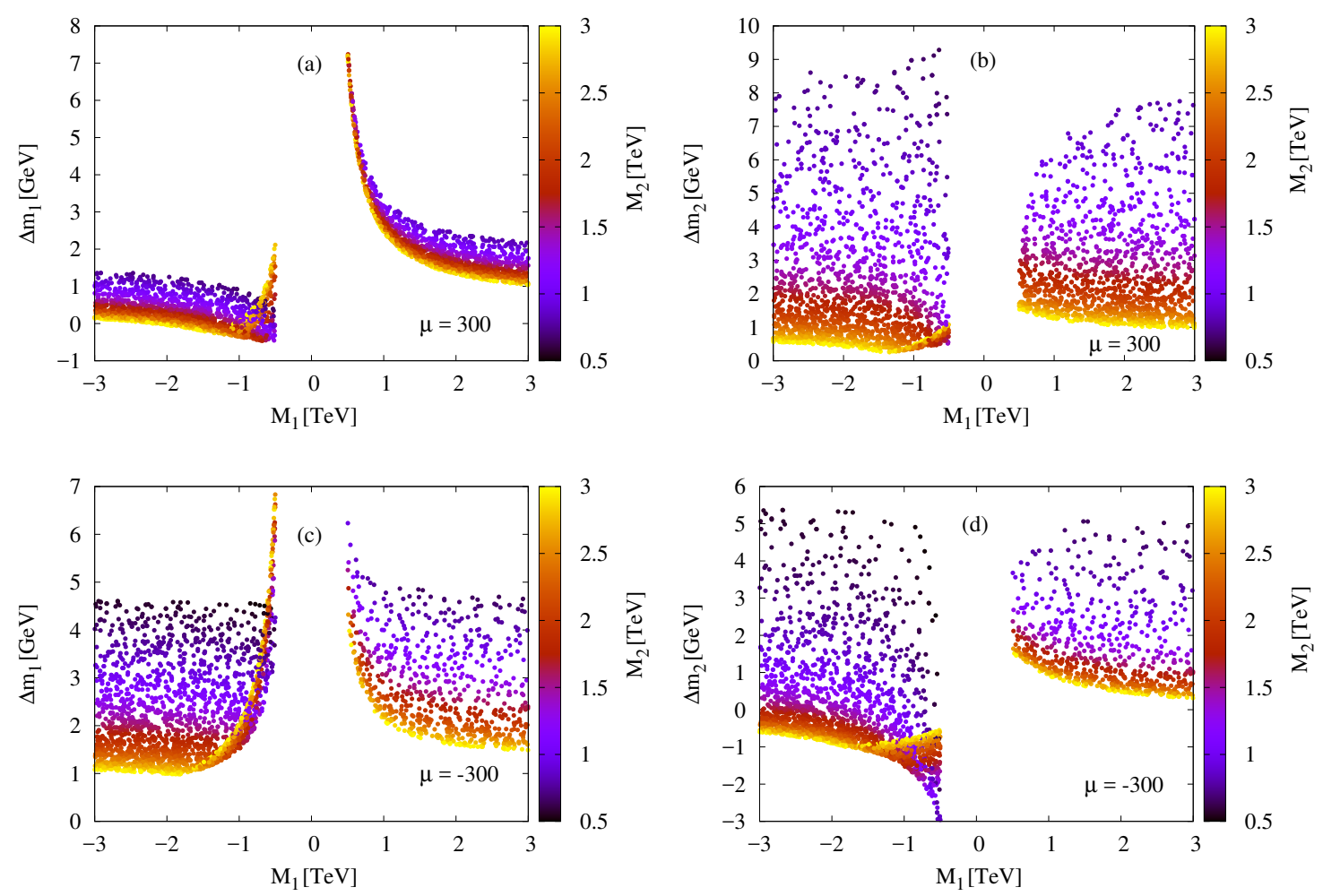

Figure 2. The left (right) panel shows the variation of the mass difference $\Delta m_{1}=m_{\tilde{\chi}_{1}^{ \pm}}-$ $m_{\widetilde{\chi}_{1}^{0}}\left(\Delta m_{2}=m_{\tilde{\chi}_{2}^{0}}-m_{\tilde{\chi}_{1}^{ \pm}}\right)$between $\tilde{\chi}_{1}^{ \pm}$and $\tilde{\chi}_{1}^{0}\left[\tilde{\chi}_{2}^{0}\right]$ for $\tan \beta=5$ with respect to $M_{1}$, with $M_{2}$ on the palette.

As shown in figure $2\left(\right.$ a), such a scenario occurs for large $M_{2}$ values $(\gtrsim 2 \mathrm{TeV})$ with $\left|M_{1}\right| \lesssim 1 \mathrm{TeV}$. Further, for $\left|M_{1}\right| \ll M_{2}$, as $\left|M_{1}\right|$ decreases one observes an upward kink in the $\Delta m_{1}$ and $\Delta m_{2}$ plots as shown in figures 2(a) and 2(b) which can be attributed to the change in nature of the lightest neutralino state from anti-symmetric to the symmetric state.

- For $\mu<0 ; M_{1}>0$ : as shown in figures $2(\mathrm{c})$ and $2(\mathrm{~d})$, similar to the $\mu>0$ case, $\Delta m_{i}$ smoothly increases with decreasing $M_{2}$ in this region as well.

- For $\mu<0 ; M_{1}<0$ : in figure 2(c) we again see (due to the change in nature of LSP) a sharp rise of $\Delta m_{1}$ for large $M_{2} \gtrsim 2 \mathrm{TeV}$ and $\left|M_{1}\right| \lesssim 1.5 \mathrm{TeV}$. Note that in this case the $\tilde{\chi}_{1}^{ \pm}$can be the heaviest higgsino-like state in a substantial region of the parameter space for $M_{2} \gtrsim 2 \mathrm{TeV}$, as shown in figure $2(\mathrm{~d})$.

\subsection{Compressed Higgsino spectrum and its decay properties}

As we have already emphasized, the focus of this work is on higgsino-like NLSPs in a scenario with a right-sneutrino LSP where the choice of small $|\mu|$ is motivated by the "naturalness" criteria $[6,8,9]$. Thus we will restrict our discussions to scenarios where the higgsino mass parameter $|\mu| \lesssim 500 \mathrm{GeV}$. The gaugino mass parameters have been assumed 

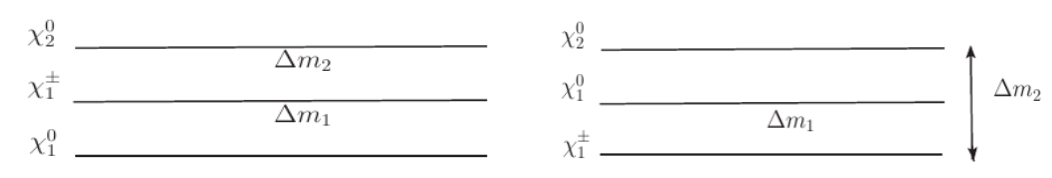

$\tilde{\nu}_{1}$

Figure 3. Schematic description of the mass spectrum with $\left|M_{1}\right|, M_{2} \gg|\mu|$, and $m_{\tilde{\nu}_{1}}<|\mu|$. Here $\left|m_{\tilde{\chi}_{2}^{0}}\right|-m_{\tilde{\chi}_{1}^{ \pm}}=\Delta m_{2}, m_{\tilde{\chi}_{1}^{ \pm}}-\left|m_{\tilde{\chi}_{1}^{0}}\right|=\Delta m_{1}$.

to be heavy for simplicity; thus the light higgsino-like states are quite compressed in mass (figure 2).

Note that since the gaugino mass parameters are much heavier, the gaugino fraction in the higgsino-like states are small $\left(\mathcal{O}\left(10^{-2}\right)\right)$. However, $M_{1}$ and $M_{2}$ play significant role in determining $\Delta m_{1}$ and $\Delta m_{2}$ and also the hierarchy between the higgsino-like states. While for most parameter space the spectra shown in the left panel of figure 3 is realized, for $M_{1}<0$ (i.e. $\operatorname{sign}\left(M_{1} M_{2}\right)=-1$ ), it is possible to achieve the chargino as the lightest higgsino-like state which leads to a spectra as shown in the right panel of figure 3. Further, with $\mu, M_{1}<0$ one can also have the chargino as the heaviest of the three higgsino-like state. However, as we will discuss subsequently in section 4 , this does not contribute to any new signature. Figure 3 schematically shows the mass hierarchies of our interest. For the electroweakinos which are dominantly higgsino-like, their production rates and subsequent decay properties would have serious implications on search strategies at accelerator machines like LHC. This in turn would play an important role in constraining the higgsino mass parameter $\mu$ in the natural SUSY framework.

We now try to briefly motivate the compositions of the LSP as well as the higgsinolike states of our interest and their decay properties. In the presence of $\tilde{\chi}_{1}^{0}$ as the lightest higgsino-like state, the decay modes available to the chargino are $\tilde{\chi}_{1}^{ \pm} \rightarrow l \tilde{\nu}_{k}^{j}$ and $\tilde{\chi}_{1}^{ \pm} \rightarrow \tilde{\chi}_{1}^{0} W^{ \pm *}$, where $j, k$ corresponds to a particular lighter sneutrino species. The partial width to the 3-body decay modes, mostly from the off-shell $W$ boson mediated processes, are suppressed by the small mass difference while small $y_{\nu}\left(\lesssim 10^{-6}\right)$ suppresses the 2body decay mode. In such a scenario, the gaugino fraction in $\tilde{\chi}_{1}^{ \pm}$, can contribute to the 2-body mode significantly in the presence of small left-right mixing $\left(\sim \mathcal{O}\left(10^{-5}\right)\right)$ in the sneutrino sector.

We illustrate the decay properies of $\tilde{\chi}_{1}^{ \pm}$based on the composition of the LSP in figure $4 .^{3}$ As shown in figure 4 , for small $T_{\nu}$ and therefore for small left admixture in

\footnotetext{
${ }^{3}$ The particular choice of gaugino mass parameters correspond to $\Delta m_{1} \lesssim 1 \mathrm{GeV}$, and the partial width in the corresponding hadronic channel is quite small $\left(\simeq 10^{-16} \mathrm{GeV}\right)$. Thus, the leptonic partial width resembles the total width of $\tilde{\chi}_{1}^{ \pm}$.
} 


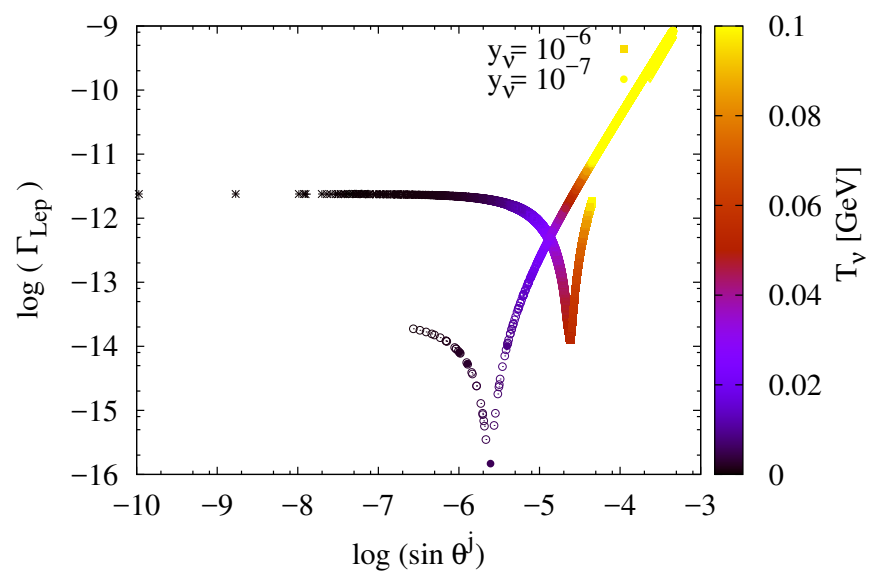

Figure 4. Variation of the partial decay width of $\widetilde{\chi}_{1}^{ \pm} \rightarrow l \widetilde{\nu}$ versus $\sin \left(\theta^{j}\right)$ in logarithmic scale for $M_{1}=-1.5 \mathrm{TeV}, M_{2}=1.8 \mathrm{TeV}$ and gaugino fraction $\sim \mathcal{O}\left(10^{-2}\right)$. Further, $M_{R}=100 \mathrm{GeV}$, $M_{L_{1 / 2}}=600 \mathrm{GeV}, m_{\tilde{\nu}}^{\text {soft }}=100 \mathrm{GeV}, \mu=300 \mathrm{GeV}$ and $\tan \beta=5$. The colored palette corresponds to $T_{\nu}$, the soft left-right mixing parameter in the sneutrino sector. The plot shows the required $T_{\nu}$ and mixing angle $\sin \left(\theta^{j}\right)$ for prompt decay of the chargino. We focus on the values of $T_{\nu}$ in our study ensuring prompt decays of the chargino.

the sneutrino sector, $y_{\nu}$ dominates the decay of $\tilde{\chi}_{1}^{ \pm}$. As $T_{\nu}$ increases past $\mathcal{O}\left(10^{-2}\right)$, the gaugino fraction plays a crucial role, which explains the rise of the partial width in the 2-body leptonic decay mode. With $y_{\nu} \sim 10^{-6}$ prompt decay of the lightest chargino to the sneutrino and lepton is always ensured. However, for $y_{\nu} \sim 10^{-7}$ prompt decay of the chargino in the leptonic channel is not viable in the absence of adequate left admixture; $T_{\nu} \gtrsim \mathcal{O}\left(10^{-2}\right) \mathrm{GeV}$ is required to ensure prompt decay in the leptonic channel. The dip in figure 4 appears as a consequence of possible cancellation between the gaugino and the higgsino contributions to the vertex factor (e.g. $\propto\left(g_{2} V_{11} \sin \theta^{j}-y_{\nu} V_{12} \cos \theta^{j}\right), g_{2}$ is the $\mathrm{SU}(2)$ gauge coupling). It is of our interest to study the scenario where the 2-body decay mode into $l \tilde{\nu}$ competes with the 3 -body decay mode. Since the present work focuses on prompt decays, we ensure small left admixture with $T_{\nu} \gtrsim \mathcal{O}\left(10^{-2}\right) \mathrm{GeV}$ in the dominantly right-sneutrino LSP to ensure prompt decay of $\tilde{\chi}_{1}^{ \pm}$in the 2-body leptonic decay mode. The mass splitting $\Delta m_{1} \gtrsim 1 \mathrm{GeV}$ has been considered to ensure a competing 3-body mode.

Since we have assumed a compressed higgsino spectrum, together with a mostly rightsneutrino LSP, the light higgsino states include $\tilde{\chi}_{1}^{0}, \tilde{\chi}_{2}^{0}, \tilde{\chi}_{1}^{ \pm}$and at least one generation of $\mathrm{CP}$-odd and/or CP-even sneutrino LSP as described in section 2. In figure 2 we showed that for a fixed $|\mu|$, the hierarchy and the mass differences between the higgsino-like states are affected significantly by the choice of the gaugino mass parameters $M_{1}, M_{2}$, and $\operatorname{sign}(\mu)$. In a similar compressed scenario within the MSSM, the higgsinos $\tilde{\chi}_{2}^{0}$ and $\tilde{\chi}_{1}^{ \pm}$decay into soft leptons or jets [99] and $\tilde{\chi}_{1}^{0}$, producing $\mathbb{E}_{T}$. Scenarios with compressed higgsinos in MSSM have been studied in the light of recent LHC data [27, 29-33]. For smaller mass differences, $130 \mathrm{MeV} \lesssim \Delta m_{1} \lesssim 2 \mathrm{GeV}$, the effective two-body process $\tilde{\chi}_{1}^{ \pm} \rightarrow \pi^{ \pm} \tilde{\chi}_{1}^{0}[100-102]$ can dominate the hadronic branching fraction. Further, when $\tilde{\chi}_{2}^{0}$ is also almost degenerate with 


\begin{tabular}{|c|c|c|c|c|c|}
\hline Parameters & $\left|M_{1}\right|(\mathrm{GeV})$ & $\left|M_{2}\right|(\mathrm{GeV})$ & $|\mu|(\mathrm{GeV})$ & $\tan \beta$ & $T_{\nu}(\mathrm{GeV})$ \\
\hline Values & $(500-3000)$ & $(500-3000)$ & 300 & 5 & 0.5 \\
\hline
\end{tabular}

Table 1. Relevant input parameters for the parameter-space scan have been presented. Other parameters kept at fixed values include: $M_{R}=100 \mathrm{GeV}, B_{M}=10^{-3} \mathrm{GeV}^{2}, M_{3}=2 \mathrm{TeV}, M_{Q_{3}}=$ $1.3 \mathrm{TeV}, M_{U_{3}}=2 \mathrm{TeV}, T_{t}=2.9 \mathrm{TeV}, M_{L_{1 / 2}}=600 \mathrm{GeV}, m_{\tilde{\nu}}^{\text {soft }}=100 \mathrm{GeV}, M_{A}=2.5 \mathrm{TeV}$, and $y_{\nu}=10^{-7}$.

$\tilde{\chi}_{1}^{ \pm}$, for an even smaller mass difference $\Delta m_{2}, \tilde{\chi}_{2}^{0} \rightarrow \gamma \tilde{\chi}_{1}^{0}$ can become significant [103-106]. Note that while the three-body decay modes (soft leptons/ jets and $\tilde{\chi}_{1}^{0}$ ) suffer from phase space suppression $\left((\Delta m)^{5}\right)$, the two-body mode $\left(\gamma \tilde{\chi}_{1}^{0}\right)$ is also suppressed by a loop factor.

In addition to the above decay channels of the compressed higgsino-like states, the present scenario with a sneutrino LSP offers additional decay channels to the lighter sneutrinos. While a $\tilde{\chi}_{1}^{0} \rightarrow \nu \tilde{\nu}$ would lead to missing transverse energy (as in the case for MSSM) without altering the signal topology if the neutralino was the LSP, $\tilde{\chi}_{1}^{ \pm} \rightarrow l \tilde{\nu}$ would have a significant impact on the search strategies. For a pure right-sneutrino LSP this decay is driven by $y_{\nu}$. In the presence of large $T_{\nu}$ and therefore a large left-right mixing in the sneutrino LSP, a gaugino fraction of $\gtrsim \mathcal{O}\left(10^{-2}\right)$ in the higgsino-like chargino begins to play a prominent role as the decay is driven by a coupling proportional to $g \delta \epsilon$ where $\delta$ represents the gaugino admixture and $\epsilon$ represents the L-R mixing in the sneutrino sector. The presence of multiple flavors of degenerate sneutrinos would lead to similar decay probabilities into each flavor and would invariably increase the branching to the two-body leptonic mode when taken together.

In the present context, as has been emphasized, only prompt decays into the leptonic channels such as $\tilde{\chi}_{1}^{ \pm} \rightarrow l \tilde{\nu}$ and $\tilde{\chi}_{i}^{0} \rightarrow \tilde{\chi}_{1}^{ \pm} j_{s} j_{s}^{\prime}$, where $j_{s}, j_{s}^{\prime}$ denote soft-jets or soft-leptons can give us a signal with one or more hard charged leptons in the final state. Since the latter consists of $\tilde{\chi}_{1}^{ \pm}$in the cascade, it can also lead to leptonic final states. These branching fractions would be affected by any other available decay channels and therefore it is important to study the different regions of parameter space for all possible decay modes of the light electroweakinos. As shown in figure 2, while in most of the parameter space $\tilde{\chi}_{1}^{0}$ is the lightest higgsino-like state, and $\tilde{\chi}_{1}^{ \pm}$is placed in between the two neutralinos (i.e. $m_{\tilde{\chi}_{1}^{0}}<m_{\tilde{\chi}_{1}^{ \pm}}<m_{\tilde{\chi}_{2}^{0}}$, it is also possible to have $\tilde{\chi}_{1}^{ \pm}$as the lightest or the heaviest higgsino-like state. The important competing modes for $\tilde{\chi}_{1}^{ \pm}$and $\tilde{\chi}_{2}^{0}$ where $m_{\tilde{\chi}_{1}^{0}}<m_{\tilde{\chi}_{1}^{ \pm}}<m_{\tilde{\chi}_{2}^{0}}$ include
(a) $\tilde{\chi}_{1}^{ \pm} \rightarrow \tilde{\chi}_{1}^{0} j_{s} j_{s}^{\prime} / \pi^{ \pm}$
(b) $\tilde{\chi}_{2}^{0} \rightarrow \tilde{\chi}_{1}^{0} j_{s} j_{s} / \gamma$
(c) $\tilde{\chi}_{2}^{0} \rightarrow \tilde{\chi}_{1}^{ \pm} j_{s} j_{s}^{\prime} / \pi^{\mp}$

where $(c)$ is usually small. However, if $\tilde{\chi}_{1}^{ \pm}$is the lightest higgsino-like state, decay modes (b) and $(c)$, together with $\tilde{\chi}_{1}^{0} \rightarrow \tilde{\chi}_{1}^{ \pm} j_{s} j_{s}^{\prime} / \pi^{\mp}$ can be present. Similarly, when $\tilde{\chi}_{1}^{ \pm}$is the heaviest higgsino-like state, decay modes $(a),(b)$ and $\tilde{\chi}_{1}^{ \pm} \rightarrow \tilde{\chi}_{2}^{0} j_{s} j_{s}^{\prime} / \pi^{ \pm}$can be present, although the latter would be sub-dominant.

In figures $5(\mu>0)$ and $6(\mu<0)$ we show the variation of branching fraction in the leptonic decay channels $\tilde{\chi}_{1}^{ \pm} \rightarrow l \tilde{\nu}_{i}$ and $\tilde{\chi}_{i}^{0} \rightarrow l \tilde{\nu}_{i} W^{*}$. The relevant parameters for the scan can be found in table 1 . 

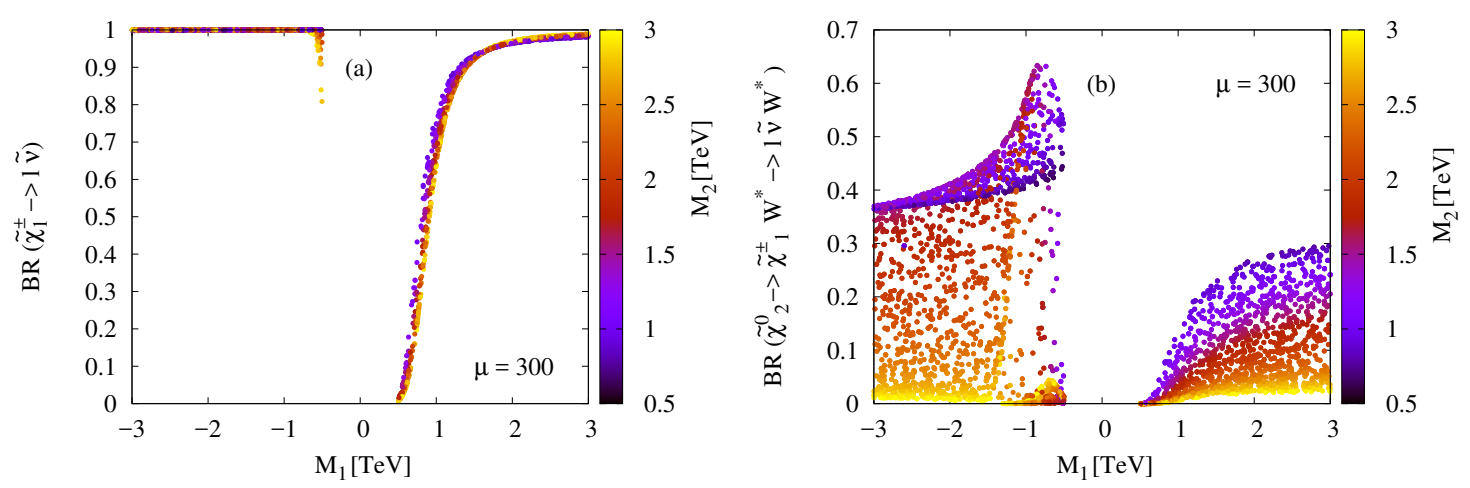

Figure 5. Variation of the leptonic branching ratios of $\widetilde{\chi}_{1}^{ \pm} \rightarrow l \widetilde{\nu}$ and $\widetilde{\chi}_{2}^{0} \rightarrow l \widetilde{\nu} W^{*}$ against the bino soft mass parameter, $M_{1}$ for the Higgsino mass parameter, $\mu=300 \mathrm{GeV}$. The wino soft mass parameter $M_{2}$ is shown in the palette.
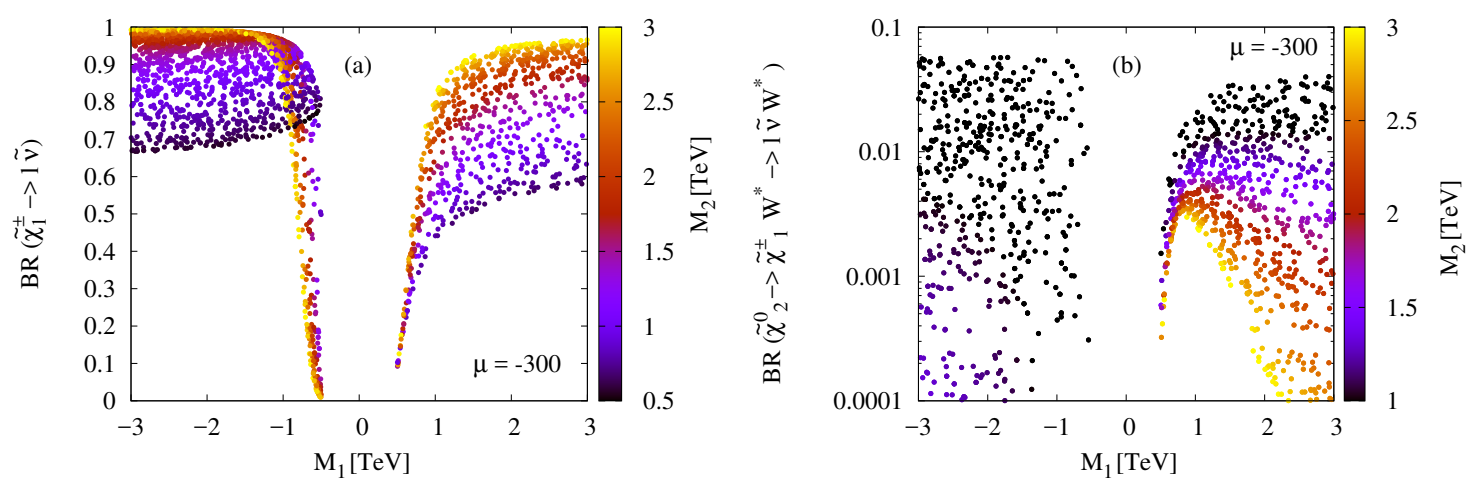

Figure 6. Variation of the leptonic branching ratios of $\widetilde{\chi}_{1}^{ \pm} \rightarrow l \widetilde{\nu}$ and $\widetilde{\chi}_{2}^{0} \rightarrow l \widetilde{\nu} W^{*}$ against the bino soft mass parameter, $M_{1}$ for the Higgsino mass parameter, $\mu=-300 \mathrm{GeV}$. The wino mass parameter $M_{2}$ is indicated in the palette.

Since the sneutrino masses and mixing matrices do not change in the scan, the two body partial decay widths $\Gamma\left(\tilde{\chi}_{1}^{ \pm} \rightarrow l \tilde{\nu}_{i}\right)$ and $\Gamma\left(\tilde{\chi}_{i}^{0} \rightarrow \nu \tilde{\nu}_{j}\right)$ are only affected by the variation of the gaugino-admixture in the higgsino-like states. However, the choice of gaugino mass parameters do affect the mass splittings $\Delta m_{1}$ and $\Delta m_{2}$ through mixing and can even alter the hierarchy. These alterations in the spectrum mostly affect the 3-body decay modes described above which has a significant effect on the branching ratio.

As shown in figure $2(\mathrm{a})$, for $\operatorname{sgn}(\mu)=+$ (i.e. $\mu=300 \mathrm{GeV}$ ) and for $M_{1}<0, \Delta m_{1}$ is almost entirely $\lesssim 1 \mathrm{GeV}$. With large $M_{2}$ and $\left|M_{1}\right| \lesssim 2 \mathrm{TeV}, \tilde{\chi}_{1}^{ \pm}$can become the lightest higgsino-like state making its leptonic branching probability close to $100 \%$ as shown in figure 5(a). However, for small $\left|M_{1}\right|$, and large $M_{2}$, where $\Delta m_{1}$ increases, this branching is somewhat reduced to about 0.8 and the 3 -body decays start becoming relevant. For $M_{1}>0$ region the branching ratio increases as $M_{1}$ increases. This can be attributed to the consistent decrease in $\Delta m_{1}$ (figure 2(a)) and therefore of the three-body partial decay width. 
Figure $5(\mathrm{~b})$ shows the variation of $\operatorname{Br}\left(\tilde{\chi}_{2}^{0} \rightarrow \tilde{\chi}_{1}^{ \pm} W^{\mp *}\right)$ as a function of $M_{1}$ and $M_{2}$. For $M_{1}<0$, generally the branching grows for larger $\Delta m_{2}$ (figure 2(b)) and decreases for smaller $M_{2}$ as the mass splitting goes down. It is again worth pointing out here that for large $M_{2}$ and with $\left|M_{1}\right| \lesssim 2 \mathrm{TeV}, \Delta m_{1}<0$ and $\tilde{\chi}_{1}^{ \pm}$becomes the lightest state. Thus in this region the three-body mode into $\tilde{\chi}_{1}^{0}$ is more phase-space suppressed compared to the decay mode into $\tilde{\chi}_{1}^{ \pm} .{ }^{4}$ Further, as $\left|M_{1}\right|$ approaches $\mu$, the symmetric state, which mixes well with the bino, acquires larger bino fraction and there can be a cancellation in the vertex factor $\propto g_{2}\left(N_{22}-\tan \theta_{W} N_{21}\right)$ for the two-body decay width into sneutrino. This can reduce the corresponding width and then increase again as $\left|M_{1}\right|$ decreases. Thus the branching ratio for the three-body decay shows a discontinuous behavior in such regions. For positive $M_{1}$, the branching ratio shows similar pattern as $\Delta m_{2}$ variation, as expected. Larger $\Delta m_{1}$ in this region implies that the three-body decay $\left(\tilde{\chi}_{2}^{0} \rightarrow \tilde{\chi}_{1}^{0} j_{s} j_{s}\right)$ can be larger, and consequently $\operatorname{Br}\left(\tilde{\chi}_{2}^{0} \rightarrow \tilde{\chi}_{1}^{ \pm} j_{s} j_{s}^{\prime}\right)$ is rather small.

For $\mu=-300 \mathrm{GeV}$, there are marked differences in the decay probabilities as the $\tilde{\chi}_{1}^{ \pm}$ can become the heaviest when $M_{1}<0$, for large regions of the parameter space in contrast to what was observed for $\mu>0$. Figure 6 (a) shows the branching ratio of $\tilde{\chi}_{1}^{ \pm} \rightarrow l \tilde{\nu}_{j}$ which decreases as $M_{2}$ increases. Although, for large $M_{2}$, the gaugino fraction in $\tilde{\chi}_{1}^{ \pm}$would be small, thus possibly reducing the partial width in this two-body decay mode; smaller $\Delta m_{1}$ in this region ensures that the competing three-body mode decreases even more. Therefore, the branching ratio in the two-body mode is enhanced. This holds true for almost the entire range of $M_{1}$. The feature in the negative $M_{1}$ region, as $\left|M_{1}\right|$ approaches $|\mu|$, where the branching ratio rises faster for larger $M_{2}$ values, corresponds to a similar fall in $\Delta m_{1}$ (see figure $2(\mathrm{c}))$.

In figure $6(\mathrm{~b})$ we show the variation of $\operatorname{Br}\left(\tilde{\chi}_{2}^{0} \rightarrow \tilde{\chi}_{1}^{ \pm} j_{s} j_{s}^{\prime}\right)$ with $M_{1}, M_{2}$. For negative $M_{1}$, this branching ratio increases with decreasing $M_{2}$, since the corresponding mass difference $\Delta m_{2}$ also increases (see figure 2). The larger $M_{2}$ values are not shown for $M_{1}<0$, since $\tilde{\chi}_{1}^{ \pm}$becomes the heaviest higgsino-like state in this region. Thus, $\Delta m_{2}<0$ as shown in see figure 2(d), and this decay mode does not contribute. For $M_{1}>0$ smaller $M_{2}$ values correspond to larger branching fractions, since $\Delta m_{2}$ becomes larger, increasing the partial width. However, for large $M_{2}$ values, the partial width decreases rapidly as $\Delta m_{2}$ decreases.

Note that $T_{\nu}=0.5 \mathrm{GeV}$ has been used in the figure. For smaller values of $T_{\nu}$ the leptonic branching ratio of $\tilde{\chi}_{1}^{ \pm}$would generally be reduced when it is not the lightest higgsino-like state. However, the generic features described above would remain similar. Note that, $y_{\nu} \sim 10^{-6}$ can lead to prompt decay even in the absence of large left-admixture, as induced by large $T_{\nu}$. Therefore, even for small $T_{\nu} \lesssim \mathcal{O}\left(10^{-2}\right)$, for certain choice of the gaugino mass parameters, the leptonic branching can be competing, and thus would be relevant to probe such scenario at collider.

\footnotetext{
${ }^{4}$ Note that, because of $\Delta m_{i} \lesssim 1.5 \mathrm{GeV}$, decay modes involving $\pi^{ \pm}$can dominate the hadronic branching fractions in this region. While we have estimated the same to be significant using routines used in SPheno-v4 [96, 97], see also refs. [100-102], the presence of large $T_{\nu}$ typically ensures that the two-body decay mode shares rather large branching fraction in these regions. In the plot we have only included three-body partial widths. A similar strategy has been adopted for regions with small $\Delta m_{2}$ as well.
} 


\section{Survey of the relevant parameter space}

We now consider the model parameter space in light of various constraints.

\subsection{General constraints}

We implement the following general constraints on the parameter-space:

- The lightest CP-even Higgs mass $m_{h}$ has been constrained within the range: $122 \leq$ $m_{h}(\mathrm{GeV}) \leq 128$ [107-109]. While the experimental uncertainty is only about $0.25 \mathrm{GeV}$, the present range of $\pm 3 \mathrm{GeV}$ is dominated by uncertainty in the theoretical estimation of the Higgs mass, see e.g. [110] and references there. ${ }^{5}$

- The lightest chargino satisfies the LEP lower bound: $m_{\tilde{\chi}_{1}^{ \pm}} \geq 103.5 \mathrm{GeV}$ [111]. The LHC bounds, which depend on the decay channels of the chargino, will be considered only for prompt channels in more detail in section 4 .

- The light sneutrino(s) (with small left-sneutrino admixture) can contribute to the non-standard decay channels of (invisible) Higgs and /or $Z$ boson. The latter requires the presence of both $\mathrm{CP}$-even and CP-odd sneutrinos below $\simeq 45 \mathrm{GeV}$. Constraints from the invisible Higgs decay $(\simeq 20 \%)$ [112] and the $\mathrm{Z}$ boson invisible width $(\simeq$ $2 \mathrm{MeV})[113]$ can impose significant constraints on the parameter space where these are kinematically allowed.

- We further impose $B_{s} \rightarrow \mu^{+} \mu^{-}[114]$ and $b \rightarrow s \gamma$ constraints [115].

\subsubsection{Implication from neutrino mass}

Recent analyses by PLANCK [116] imposes the following constraint on the neutrino masses: $\sum m_{\nu}^{i} \lesssim 0.7 \mathrm{eV}$. In the present scenario, the neutrinos can get a tree-level mass, as is usual in the Type-I see-saw scenario. For $y_{\nu} \sim 10^{-6}$, and $M_{R} \sim 100 \mathrm{GeV}$, the active neutrino mass is of $\mathcal{O}(0.1) \mathrm{eV}$. Further, as discussed in section 2.1, a non-zero Majorana mass term $M_{R}$, and the corresponding soft-supersymmetry breaking term $B_{M}$ introduce a splitting between the CP-even and CP-odd mass eigenstates of right-sneutrinos. In the presence of sizable left-right mixing, significant contribution to the Majorana neutrino mass can be generated at one-loop level in such a scenario, the details depend on the gaugino mass parameters $[80,87]$. Thus, regions of large $B_{M}$, in the presence of large left-right mixing in the sneutrino sector (induced by a large $T_{\nu}$ ) can be significantly constrained from the above mentioned bound on (active) neutrino mass. In figure 7 we show the allowed region in the $T_{\nu}-B_{M}$ plane. We consider $y_{\nu} \in\left\{10^{-6}, 10^{-7}\right\}$ while the other parameters are fixed as follows: $\mu=300 \mathrm{GeV}, M_{3}=2 \mathrm{TeV}, M_{Q_{3}}=1.5 \mathrm{TeV}, T_{t}=2.9 \mathrm{TeV}, M_{L_{1 / 2}}=600 \mathrm{GeV}$, $m_{\tilde{\nu}}^{\text {soft }}=100 \mathrm{GeV}$ and $M_{A}=2.5 \mathrm{TeV}$. While in the former case the tree-level and radiative contributions to the neutrino mass can be comparable (with each being $\mathcal{O}(0.1) \mathrm{eV}$ ), the radiative corrections often dominate for the latter. As shown in the figure, clearly larger $T_{\nu}$ values are consistent with neutrino mass for smaller $B_{M}$.

\footnotetext{
${ }^{5}$ Note that, besides the MSSM contributions, rather large $T_{\nu}$ can induce additional contributions to the Higgs mass [66]. Our numerical estimation takes this effect into account.
} 


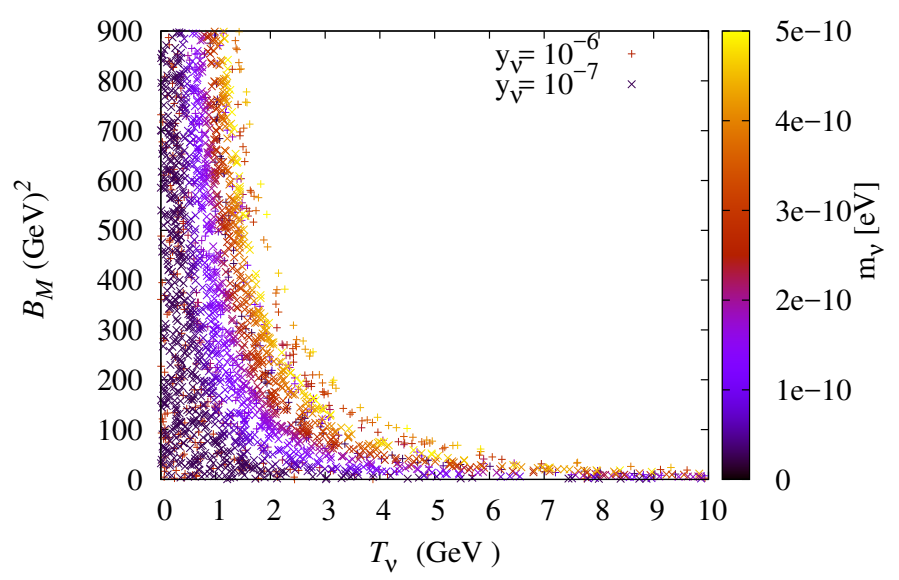

Figure 7. Allowed regions of $B_{M}$ and $T_{\nu}$ plane for $M_{1}=1.5 \mathrm{TeV}, M_{2}=1.8 \mathrm{TeV}$ and gaugino fraction $\sim \mathcal{O}\left(10^{-2}\right)$. The colored palette denotes the mass of the heaviest neutrino.
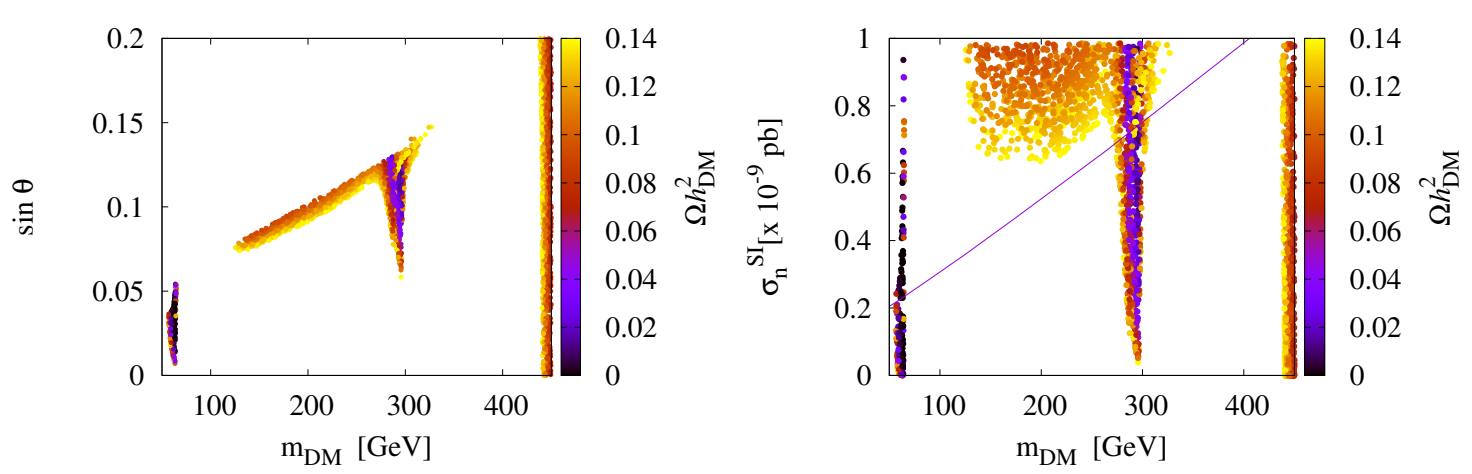

Figure 8. In the left panel, the dependence of the relic abundance of $\tilde{\nu}_{1}$ has been shown on its mass and left-fraction. The right panel shows the allowed region respecting the direct detection constraint from XENON-1T.

\subsubsection{Implications for dark matter}

Within the paradigm of standard model of cosmology the relic abundance is constrained as $0.092 \leq \Omega h^{2} \leq 0.12$ [116]. Stringent constraints from direct search constraints require the DM-nucleon (neutron) interaction to be less than about $10^{-9} \mathrm{pb}$, which varies with the mass of DM, see e.g. LUX [117], PANDA-II [118] and Xenon-1T [119].

In figure 8, with $y_{\nu} \in\left\{10^{-6}, 10^{-7}\right\}, \tan \beta \in\{5,10\}, \mu=-450 \mathrm{GeV}, m_{A}=600 \mathrm{GeV}$ and all other relevant parameters are fixed as before, we scan over the set of parameters $\left\{T_{\nu}, m_{\tilde{\nu}}, B_{M}\right\}$ (first generation only). We plot the left-admixture $\left(\sin \theta^{j}\right)$ in the LSP required to obtain the thermal relic abundance and direct detection cross section against its mass in the left and the right panel respectively. We have used micrOMEGAs-3.5.5 [120] to compute the thermal relic abundance and direct detection cross-sections. 
With $T_{\nu} \gtrsim \mathcal{O}\left(10^{-3}\right)$, which is the region of interest to allow left-right mixing in the sneutrino sector, the right-sneutrino LSP thermalizes with the (MS)SM particles via its interaction with left-sneutrino and Higgs bosons. The important annihilation processes involve s-channel processes mediated by Higgs bosons, as well as four-point vertices leading to $h h, W^{ \pm} W^{\mp}, Z Z, t \bar{t}$ final states. However, large left-right mixing induces large direct detection cross-section. In figure 8 we have only shown parameter regions with a mass difference of at least $1 \mathrm{MeV}$ between the CP-even and the CP-odd states to prevent the $Z$ boson exchange contribution to the direct detection $[59,121,122] .{ }^{6}$ There are t-channel contributions mediated by Higgs bosons, mostly from the D-term, as well as the tri-linear term $T_{\nu}$, and is proportional to the left-right mixing $(\sin \theta)$ in the sneutrino sector. Note that, we have only shown points with spin-independent direct detection cross-section less than $10^{-9} \mathrm{pb}$.

As shown in the left panel of figure $8, \sin \theta$ of $\mathcal{O}(0.1)$ is required to achieve the right thermal relic abundance. The right relic abundance is achieved soon after the dominant annihilation channels into the gauge bosons (and also Higgs boson) final states are open (i.e. $\left.m_{\mathrm{DM}} \gtrsim 130 \mathrm{GeV}\right)$, while at the Higgs resonances $\left(m_{h}=125 \mathrm{GeV}\right.$ and $\left.m_{A} \sim 600 \mathrm{GeV}\right)$ a lower admixture can be adequate. Further, co-annihilation with the low-lying higgsinolike states $(|\mu| \sim 450 \mathrm{GeV})$, when the LSP mass is close to $450 \mathrm{GeV}$, can also be effective. As shown in the right panel of the same figure, for $m_{\mathrm{DM}} \lesssim 450 \mathrm{GeV}$, most parameter space giving rise to the right thermal relic abundance is tightly constrained from direct searches (spin-independent cross-sections) from Xenon-1T [119] (similar constraints also arise from LUX [117], PANDA-II [118]), the exceptions being the resonant annihilation and co-annihilation regions.

Note that for very small $T_{\nu}$ and $y_{\nu} \lesssim 10^{-6}$, the effective interaction strength of rightsneutrinos may be smaller than the Hubble parameter at $T \simeq m_{\mathrm{DM}}$. In such a scenario, non-thermal production, especially from the decay of a thermal NLSP, can possibly generate the relic abundance [60-63]. Further, non-thermal productions can also be important in certain non- standard cosmological scenarios, e.g. early matter domination or low reheat temperature, see e.g. [123, 124]. In addition, large thermal relic abundance can be diluted if substantial entropy production takes place after the freeze-out of the DM. For such regions of parameter space our right-sneutrino LSP is likely to be a non-thermal DM candidate.

\section{Signatures at $\mathrm{LHC}$}

We now focus on the LHC signal of the higgsino-like electroweakinos in the presence of a right-sneutrino LSP. As discussed, the various decay modes available to $\widetilde{\chi}_{1}^{ \pm}, \widetilde{\chi}_{2}^{0}$ and $\widetilde{\chi}_{1}^{0}$ in presence of a right-sneutrino LSP depend not only on the mixing among the various sparticle components but also crucially on the mass splittings. The LHC signals would

\footnotetext{
${ }^{6}$ We have checked that with $1 \mathrm{MeV}$ mass splitting and a left-admixture of $\mathcal{O}\left(10^{-} 2\right)$, as is relevant for thermal relic, the heavier of the $\mathrm{CP}$-even and the $\mathrm{CP}$-odd state has a decay width of $\sim 10^{-20} \mathrm{GeV}$, mostly into the LSP and soft leptons/quarks via off-shell $Z$ boson. This corresponds to a lifetime of $\lesssim 10^{-3} \mathrm{~s}$. Thus it would decay well before the onset of Big Bang Nucleosynthesis (BBN) and is consistent with constraints from the same.
} 
then reflect upon the above dependencies on the parameter space. We therefore look at all possible signals for different regions of $\Delta m_{1 / 2}$ and $T_{\nu}$. While there are regions of $\Delta m_{1 / 2}$ where the chargino decays non-promptly to pions that lead to the chargino traveling in the detector for some length and then decay into a soft pion and neutralino. In such cases, since both decay products are invisible, the relevant search channel at LHC is the disappearing tracks $[32,33,125]$. Our focus however, is primarily on the prompt decay of the chargino to hard leptons (small $\Delta m_{1 / 2}$ and large $T_{\nu}$ ) which would be clean signals to observe at LHC.

The following production channels are of interest to us:

$$
p p \rightarrow \widetilde{\chi}_{1}^{ \pm} \tilde{\chi}_{2}^{0}, \widetilde{\chi}_{1}^{ \pm} \tilde{\chi}_{1}^{0}, \tilde{\chi}_{1}^{+} \tilde{\chi}_{1}^{-}, \tilde{\chi}_{1}^{0} \tilde{\chi}_{2}^{0}, \tilde{\chi}_{1}^{0} \tilde{\chi}_{1}^{0}, \tilde{\chi}_{2}^{0} \tilde{\chi}_{2}^{0}, \widetilde{l} \widetilde{l}, \widetilde{l} \widetilde{l}^{*}, \widetilde{l} \widetilde{\nu}, \widetilde{\nu} \widetilde{\nu}
$$

where the sleptons and sneutrinos are heavier than the electroweakinos here. The LSP pair production is excluded in the above list. The processes as given in eq. (4.1) are in decreasing order of production cross-sections as obtained from Prospino [126-128]. The associated chargino neutralino pair, i.e $\widetilde{\chi}_{1}^{ \pm} \widetilde{\chi}_{1 / 2}^{0}$ production has the largest cross-section followed by the chargino pair production, $\widetilde{\chi}_{1}^{+} \widetilde{\chi}_{1}^{-}$as in figure 9 . In the pure higgsino limit, the pair production cross-section of $\widetilde{\chi}_{1}^{0} \widetilde{\chi}_{1}^{0}$ and $\widetilde{\chi}_{2}^{0} \widetilde{\chi}_{2}^{0}$ are negligible compared to the other processes. Since the strong sector is kept decoupled and the compressed higgsino sector leads to soft jets and leptons, the only source of hard jets are from initial-state radiations (ISR). The suppressed jet multiplicity in the signal could prove to be a potent tool for suppressing SM leptonic backgrounds coming from the strongly produced $t \bar{t}$ and single top subprocesses which would give multiple hard jets in the final state in association with the charged leptons. Therefore we shall focus on the following leptonic signals with low hadronic activity:

- Mono-lepton $+\leq 1$ jet $+\mathbb{E}_{T}$

- Di-lepton +0 jet $+\mathbb{E}_{T}$

The mono-lepton signals would come from the pair production of $\widetilde{\chi}_{1}^{+} \widetilde{\chi}_{1}^{-},\left(\widetilde{\chi}_{1}^{ \pm} \rightarrow l \widetilde{\nu}\right.$ and $\left.\widetilde{\chi}_{1}^{ \pm} \rightarrow \widetilde{\chi}_{1}^{0} W^{* \pm}\right)$, and associated pair production, $\widetilde{\chi}_{1}^{ \pm} \widetilde{\chi}_{i}^{0}$ with $i=1,2\left(\widetilde{\chi}_{1}^{ \pm} \rightarrow l \widetilde{\nu}\right.$ and $\widetilde{\chi}_{i}^{0} \rightarrow$ $\nu \widetilde{\nu})$. A smaller contribution also comes from the production of $\widetilde{\chi}_{1}^{0} \widetilde{\chi}_{2}^{0}\left(\widetilde{\chi}_{2 / 1}^{0} \rightarrow \widetilde{\chi}_{1}^{ \pm} W^{* \mp}\right.$ and $\widetilde{\chi}_{1 / 2}^{0} \rightarrow \nu \widetilde{\nu}$ ) leading to missing energy. Among the di-lepton signals we look into both opposite sign leptons and same sign lepton signal with missing energy. Opposite sign leptons arise from the pair produced $\widetilde{\chi}_{1}^{ \pm} \widetilde{\chi}_{1}^{ \pm}$, with the chargino decaying leptonically as $\widetilde{\chi}_{1}^{ \pm} \rightarrow l \widetilde{\nu}$. In regions of the parameter space where $\widetilde{\chi}_{2 / 1}^{0}$ is heavier than $\widetilde{\chi}_{1}^{ \pm}, \widetilde{\chi}_{1}^{ \pm} \widetilde{\chi}_{i}^{0}, i=1,2$ process may contribute to the di-lepton state via $\widetilde{\chi}_{i}^{0} \rightarrow \widetilde{\chi}_{1}^{ \pm} W^{* \mp}$ followed by $\widetilde{\chi}_{1}^{ \pm} \rightarrow l \widetilde{\nu}$. In such cases, there could be either opposite sign di-lepton signal or same-sign di-lepton signal owing to the Majorana nature of the neutralinos $\left(\widetilde{\chi}_{i}^{0}\right)$. A similar contribution to both channels come from $\widetilde{\chi}_{1}^{0} \widetilde{\chi}_{2}^{0}$ with $\widetilde{\chi}_{1 / 2}^{0} \rightarrow \widetilde{\chi}_{1}^{ \pm} W^{* \mp}$. Also there are sub-leading contributions from slepton pair productions which can become relevant if light sleptons are also present in the spectrum.

It is worth pointing out that in very particular regions of the parameter space $\widetilde{\chi}_{1}^{ \pm}$ is the NLSP and therefore always decays to a hard lepton and sneutrino LSP. In such cases, signal rates for the di-lepton channel would be most interesting and dominant rates for same-sign di-lepton would be a particularly clean channel which will be important to 


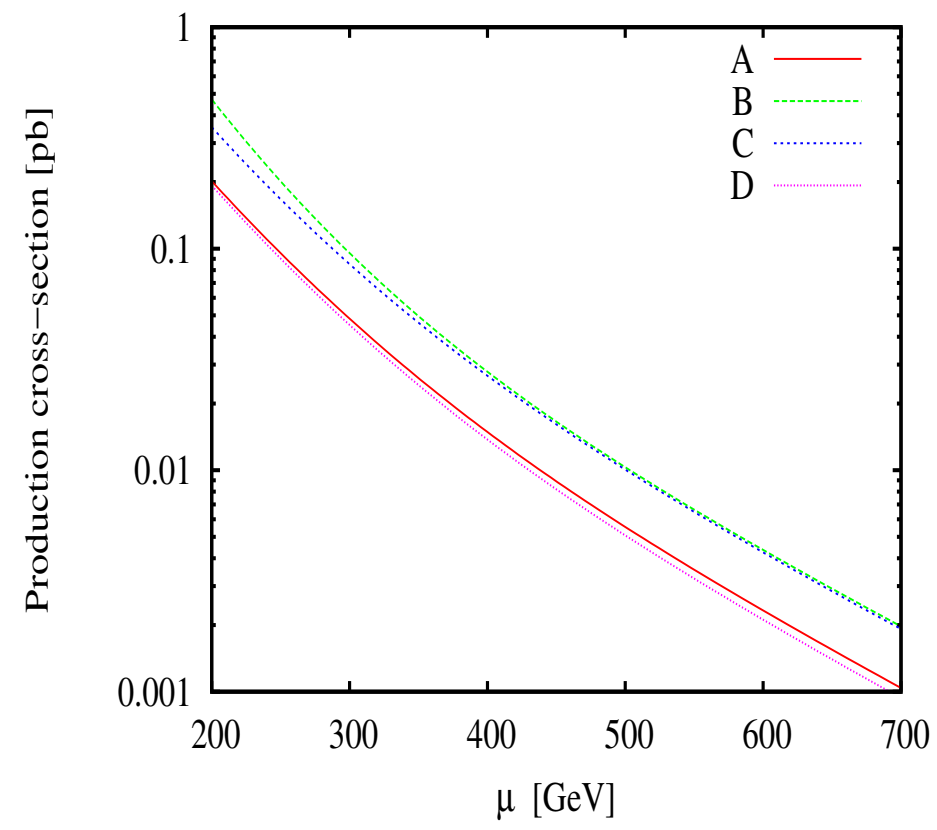

Figure 9. LO cross-sections of the different production channels at $\sqrt{s}=13 \mathrm{TeV}$. Here, $\mathrm{A}=\widetilde{\chi}_{1}^{+} \widetilde{\chi}_{1}^{-}$, $\mathrm{B}=\widetilde{\chi}_{1}^{ \pm} \widetilde{\chi}_{1}^{0}, \mathrm{C}=\widetilde{\chi}_{1}^{ \pm} \widetilde{\chi}_{2}^{0}$ and $\mathrm{D}=\widetilde{\chi}_{1}^{0} \widetilde{\chi}_{2}^{0} . \widetilde{t}_{1}$ and $\widetilde{b}_{1}$ are of mass $\sim 1.4 \mathrm{TeV}$. The NLO cross-sections can be estimated by using a $\mathrm{K}$ factor $\sim 1.25$.

probe high values of $\mu$ very effectively. This is very particular of the parameter region when $M_{1}$ is negative and one has a sneutrino LSP.

\subsection{Constraints on electroweakino sector from LHC}

Before setting up our analysis on the above signals we must consider the role of existing LHC studies that may be relevant for constraining the parameter space of our interest. LHC has already looked for direct production of lightest $\widetilde{\chi}_{1}^{ \pm}, \widetilde{\chi}_{2}^{0}$ and $\widetilde{\chi}_{1}^{0}$ in both run 1 and run 2 searches at 7,8 and $13 \mathrm{TeV}$ respectively albeit assuming simplified models. Search results have been reinterpreted both in terms of non-prompt as well as prompt decays of the Higgsinos. Since the focus of our study is on prompt decays of $\widetilde{\chi}_{1}^{ \pm}$, we consider the prompt search results only.

The LHC results have been reinterpreted assuming simplified models with and without intermediate left and right sleptons with $\widetilde{\chi}_{1}^{0}$ LSP contributing to $\mathbb{E}_{T}$ (see table 3 ).

- Assuming 100\% leptonic branching of the sparticles and an uncompressed spectra, CMS has ruled out degenerate wino-like $m_{\widetilde{\chi}_{1}^{ \pm}}, m_{\widetilde{\chi}_{2}^{0}}<1.2 \mathrm{TeV}$ for a bino-like $m_{\widetilde{\chi}_{1}^{0}}<$ $600 \mathrm{GeV}$ from same-sign di-lepton, three lepton and four lepton searches with at most 1 jet [129]. The limits vary slightly depending on the choice of slepton masses. For the nearly compressed higgsino sector and assuming mass degeneracy of the lightest chargino $\left(\widetilde{\chi}_{1}^{ \pm}\right)$and next-to-lightest neutralino $\left(\widetilde{\chi}_{2}^{0}\right)$ the alternate channels probed by LHC are soft opposite sign di-leptons searches [130]. The mass limits on the compressed higgsino sector relax to $\sim 230(170) \mathrm{GeV}$ for $m_{\widetilde{\chi}_{1}^{0}} \sim 210(162) \mathrm{GeV}$. 
- ATLAS has also extensively looked for compressed higgsinos in opposite sign dilepton and trilepton final states excluding $m_{\widetilde{\chi}_{2}^{0}} \sim 150 \mathrm{GeV}$ for splittings as low as $\Delta m\left(\widetilde{\chi}_{2}^{0}-\right.$ $\left.\tilde{\chi}_{1}^{0}\right)=3 \mathrm{GeV}$ while the limit further improves by $20 \mathrm{GeV}$ for degenerate $\widetilde{\chi}_{2}^{0}$ and $\widetilde{\chi}_{1}^{ \pm}$[131]. For the uncompressed case, searches with low hadronic activity [132] look for di-lepton and trilepton signal with upto 0 or 1 jet. The di-lepton search with no jet from chargino pair production, with charginos decaying to the neutralino LSP via intermediate sleptons sets a limit on the $m_{\widetilde{\chi}_{1}^{ \pm}}>720 \mathrm{GeV}$ for $m_{\widetilde{\chi}_{1}^{0}}<200 \mathrm{GeV}$. The trilepton channel excludes mass degenerate $m_{\widetilde{\chi}_{2}^{0}}, m_{\widetilde{\chi}_{1}^{ \pm}} \sim 1.15 \mathrm{TeV}$ for $m_{\widetilde{\chi}_{1}^{0}}$ up to $580 \mathrm{GeV}$.

In table 2, we list all relevant searches implemented in the public reinterpretation software CheckMATE. The ones, not implemented in CheckMATE have been recast in Madanalysis-v5 as shown in table 3 and benchmarks have been chosen to pass all the relevant searches. Alternate results from LHC which constrain the compressed higgsino sector is the monojet $+\mathbb{E}_{T}$ channel [160]. However no limits on the electroweak sector are yet placed from it. We ensure that our chosen benchmarks pass all of these discussed analyses.

\subsection{Impact of additional related searches at LHC}

For monolepton signal, multiple searches in both ATLAS and CMS look for single lepton final states with multiple (b) jets (refer table 2 B.5) thus focussing on the production of coloured sparticles. However, there are not many dedicated SUSY search results for exploring low hadronic jet activity and low $\mathbb{E}_{T}$. One of the closest analysis is [163], however employing a large cut on $\mathbb{E}_{T}>300 \mathrm{GeV}$. Such a large cut on missing energy depletes the SUSY signal for low higgsino masses, ie., $\mu \sim 300-500 \mathrm{GeV}$. Both ATLAS and CMS have also looked into resonant searches for heavy gause bosons and considered monoleptonic channels with missing energy and placed limits on mass of heavy gauge bosons [161, 162]. Since no reinterpretation exists on SUSY models in run 1 or run 2 so far, we reinterpret and take into account these limits in the context of our study and impose any constraints that apply on our parameter space. Few studies on soft leptons [163, 172], involve high cuts on missing transverse energy $\left(\mathbb{E}_{T}\right)$ and hadronic energy $\left(H_{T}\right)$ even though requiring upto two light jets and atmost one $b$ jet. It is important to point out that since the signals from the higgsino sector here are devoid of sources of $\mathrm{b}$ jets, and only ISR jets are present, signal efficiency reduce substantially with cuts on large values of hadronic energy and hard $\mathrm{b}$ jet requirements. Further, owing to a light $\mu$ parameter, and consequently a low-lying higgsino states, a large missing energy cut of $\sim \mu$ reduces signals significantly. Hence, these searches would require large luminosity to probe the compressed higgsino sector. Reducing the hard cuts on $\mathbb{E}_{T}$, b-veto and number of jets coupled with hadronic energy would allow better sensitivity to such signals and we have attempted to give an estimate of the results for the run 2 of LHC.

For di-lepton final states, both ATLAS and CMS have looked at stop searches or gluino searches giving rise to opposite sign or same sign leptons accompanied with multiple jets and $\mathrm{b}$ jets along with missing energy $[173,174]$. As argued above, these searches weakly constrain the scenario we are interested in this study. However there are some searches 


\begin{tabular}{|c|c|c|c|c|}
\hline $\mathbf{A}$ & \multicolumn{4}{|l|}{$\sqrt{s}=8 \mathrm{TeV}$} \\
\hline S.No. & Final State & Luminosity (in fb ${ }^{-1}$ ) & ATLAS & CMS \\
\hline 1 & 3 leptons $+\mathbb{E}_{T}$ & 20.3 & {$[133]$} & - \\
\hline 2 & Stop search with 2 leptons $+\mathbb{E}_{T}$ & 20.3 & {$[134]$} & - \\
\hline 3 & Stop search with $\mathrm{Z}$ boson and b jets $+\mathbb{E}_{T}$ & 20.3 & {$[135]$} & - \\
\hline 4 & 2 same-sign leptons or 3 leptons $+\mathbb{E}_{T}$ & 20.3 & {$[136]$} & - \\
\hline 5 & 1 lepton $+(\mathrm{b})$ jets $+\mathbb{E}_{T}$ & 20.3 & {$[137]$} & - \\
\hline 6 & 2 leptons + jets $+\mathbb{E}_{T}$ & 20.3 & {$[138]$} & - \\
\hline 7 & Monojet $+\mathbb{E}_{T}$ & $20.3,19.5$ & {$[139]$} & [140] \\
\hline 8 & 2 leptons $+2 \mathrm{~b}$ jets $+\mathbb{E}_{T}$ & 20.3 & {$[141]$} & - \\
\hline 9 & 1 lepton $+\geq 4$ jets $+\mathbb{E}_{T}$ & 20.5 & {$[142]$} & - \\
\hline 10 & 3 leptons $+\mathbb{E}_{T}$ & 20.3 & {$[143]$} & - \\
\hline 11 & 2 leptons $+\mathbb{E}_{T}$ & 20.3 & {$[144]$} & - \\
\hline 12 & $0-1$ lepton $+\geq 3$ b jets $+\mathbb{E}_{T}$ & 20.1 & {$[145]$} & - \\
\hline 13 & 2 leptons + jets $+\mathbb{E}_{T}$ & $20.3,19.5$ & {$[146]$} & {$[147]$} \\
\hline 14 & 1 lepton $+\geq 3$ jets $+\geq 1 \mathrm{~b}$ jet $+\mathbb{E}_{T}(\mathrm{DM}+2$ top $)$ & 19.7 & - & [148] \\
\hline 15 & Opposite sign leptons $+3 \mathrm{~b}$ tags & 19.5 & - & [149] \\
\hline B & \multicolumn{4}{|l|}{$\sqrt{s}=13 \mathrm{TeV}$} \\
\hline S.No. & Final State & Luminosity (in fb ${ }^{-1}$ ) & ATLAS & CMS \\
\hline 1 & 2 same-sign or 3 leptons + jets $+\mathbb{E}_{T}$ & 3.2 & {$[150]$} & - \\
\hline 2 & Mono jet $+\mathbb{E}_{T}$ & 3.2 & {$[151]$} & - \\
\hline 3 & 1 lepton + jets $+\mathbb{E}_{T}$ & 3.3 & {$[152]$} & - \\
\hline 4 & $0-1$ lepton $+3 \mathrm{~b}$ jets $+\mathbb{E}_{T}$ & 3.3 & {$[153]$} & - \\
\hline 5 & 1 lepton $+(\mathrm{b})$ jets $+\mathbb{E}_{T}$ & 3.2 & {$[154]$} & - \\
\hline 6 & 2 leptons $(\mathrm{Z})+$ jets $+\mathbb{E}_{T}$ & 3.2 & {$[155]$} & - \\
\hline 7 & 1 lepton + jets & 3.2 & {$[156]$} & \\
\hline 8 & 2 leptons + jets $+\mathbb{E}_{T}$ & 13.3 & {$[157]$} & - \\
\hline 9 & 1 lepton $+(\mathrm{b})$ jets $+\mathbb{E}_{T}$ & 13.2 & {$[158]$} & - \\
\hline 10 & 2 leptons + jets $+\mathbb{E}_{T}$ & 2.2 & - & [159] \\
\hline
\end{tabular}

Table 2. List of LHC analyses at $\mathrm{s}=8,13 \mathrm{TeV}$ implemented in the public software CheckMATE. All the benchmarks considered in our study pass these analyses, without showing any excess above the observed number of events at 95\% CL.

specifically for soft leptons studied in $8 \mathrm{TeV}$ [172] against which we check our benchmarks using Madanalysis-v5. For opposite sign dileptons, important searches from LHC which constrain our scenario are from ATLAS [132] and CMS [165]. Among other kinematic variables such as high lepton pt cuts, both studies focus on using large cuts on $M_{T_{2}}$ ( $\geq$ $90 \mathrm{GeV})$. We implement these analyses in Madanalysis-v5 and choose benchmarks such that they are not excluded by current data. For same sign dilepton, the most constraining limit comes from CMS [167] with atmost 1 jet. Other searches usually focus on the strong sector thus requiring large number of jets. 


\begin{tabular}{|c|c|c|c|}
\hline Final State & ATLAS & CMS & Madanalysis-v5 \\
\hline 1 lepton $+\mathbb{E}_{T}$ & {$[161]$} & {$[162,163]$} & Yes \\
2 leptons $+\mathbb{E}_{T}$ & {$[131,132]$} & {$[164-166]$} & Yes \\
2 same-sign leptons $+\mathbb{E}_{T}$ & - & {$[167]$} & Yes \\
3 or more leptons $+\mathbb{E}_{T}$ & {$[132,168,169]$} & {$[167,170,171]$} & Not relevant for this study \\
\hline
\end{tabular}

Table 3. Leptonic searches at $\sqrt{s}=13 \mathrm{TeV}$ LHC with few jets (i,e, $\left.N_{j} \leq 2\right)$, as relevant for this study.

\subsection{Benchmarks}

In the context of natural supersymmetry with degenerate first and second generation sneutrino as LSP, we look into regions of parameter space allowed by neutrino physics constraints, LHC data and direct detection cross-section constraints. We select five representative points of the parameter space and analyze their signal at the current run of LHC. We check the viability of the chosen benchmarks for multi-leptonic signatures by testing the signal strengths against existing experimental searches implemented in the public software CheckMATE $[175,176]$. Amongst the searches implemented in CheckMATE, mono-jet along with missing energy search [151] provides the most stringent constraint. Among the other $13 \mathrm{TeV}$ searches as listed in table 3, same-sign di-lepton and opposite-sign di-lepton searches also impose a stringent constraint on the current scenario. The allowed same-sign di-lepton branching is restricted to $4 \%$ or lower for $\mu=300 \mathrm{GeV}$ for uncompressed scenarios. A higher value of $\mu$ and hence a lower production cross-section allows a larger same-sign di-lepton branching thereby allowing us to probe a wider range of the parameter space.

We choose parameters with $|\mu|$ in the range $300-500 \mathrm{GeV}, M_{1 / 2} \sim 2 \mathrm{TeV}$ and $\tan \beta \sim 5-10 \mathrm{GeV}$ as listed in table 4 . The choice of the benchmarks ensure prompt decay of the chargino to a hard lepton and LSP, i.e., $\Gamma>10^{-13} \mathrm{GeV}$. The gaugino mass parameters $M_{1}$ and $M_{2}$ are large such that the spectrum consists of two light higgsino-like neutralinos $\widetilde{\chi}_{1}^{0}, \widetilde{\chi}_{2}^{0}$ and a nearly degenerate light higgsino-like $\widetilde{\chi}_{1}^{ \pm}$within $\mathcal{O}(2-4) \mathrm{GeV}$. However there is considerable amount of freedom in choosing the relative sign among the soft parameters $M_{1}, M_{2}$ and $\mu$. Both the first and second generation squarks as well as gluino soft mass parameter are set to $\sim 2 \mathrm{TeV}$. The stops are also kept heavy to ensure the light CP-even Higgs mass and signal strengths to be within the allowed experimental values. Both the first two generation left and right sleptons are kept above the higgsino sector and when possible within the reach of $\mathrm{LHC}$, in the range $360-600 \mathrm{GeV}$, in the different benchmark points studied. Following our discussion in section 2.2 on the $M_{1}-M_{2}$ dependence of the masses, the benchmarks represent points in the following regions of parameter space:

- Region A: $M_{1}>0, M_{2}>0$ and $\mu>0$, with $\widetilde{\chi}_{1}^{0}$ as $\operatorname{NLSP}(\mathbf{B P 1})$.

- Region B: $M_{1}>0, M_{2}>0$ and $\mu<0$, with $\tilde{\chi}_{1}^{0}$ as NLSP (BP2-a and BP2-b).

- Region C: $M_{1}<0, M_{2}>0$ and $\mu>0$, with $\widetilde{\chi}_{1}^{ \pm}$as NLSP (BP3 and BP4).

BP1 represents a point in the $M_{1} M_{2}>0$ and $\mu>0$ plane with $M_{1}=1.5 \mathrm{TeV}$, $M_{2}=1.8 \mathrm{TeV}, \tan \beta=5$ and $\Delta m_{1 / 2} \sim 2 \mathrm{GeV}$. The LSP mass is $\sim 140 \mathrm{GeV}$ and therefore 


\begin{tabular}{|c|c|c|c|c|c|}
\hline Parameters & BP1 & BP2-a & BP2-b & BP3 & BP4 \\
\hline$\mu$ & 300 & -500 & -300 & 300 & 400 \\
\hline $\tan \beta$ & 5 & 5 & 10 & 5 & 6.1 \\
\hline$M_{1}$ & 1500 & 1500 & 2000 & -860 & -1150 \\
\hline$M_{2}$ & 1800 & 1800 & 1000 & 2500 & 2500 \\
\hline$M_{3}$ & 2000 & 2000 & 2000 & 2000 & 2000 \\
\hline$M_{A}$ & 2500 & 803.2 & 2500 & 2500 & 2500 \\
\hline$T_{t}$ & 2900 & 2900 & -2500 & 2950 & 2750 \\
\hline$M_{R}$ & 100 & 100 & 100 & 100 & 100 \\
\hline$B M_{R}\left(\mathrm{GeV}^{2}\right)$ & $10^{-3}$ & 10.7 & 143 & $10^{-3}$ & $10^{-3}$ \\
\hline$m_{\widetilde{\nu}}$ & 100 & 404 & 245 & 245 & 316.2 \\
\hline$Y_{\nu}\left(\times 10^{-7}\right)$ & 1 & 10 & 1 & 1 & 1 \\
\hline$T_{\nu}$ & 0.02 & 140 & 0.8 & 4.0 & 0.06 \\
\hline$m_{\widetilde{\chi}_{1}^{ \pm}}$ & 303.6 & 510.9 & 307.5 & 305.4 & 407.2 \\
\hline$m_{\widetilde{\chi}_{1}^{0}}$ & 301.7 & 508.4 & 303.5 & 305.5 & 407.3 \\
\hline$m_{\widetilde{\chi}_{2}^{0}}$ & 305.8 & 512.2 & 311.5 & 305.8 & 407.5 \\
\hline$m_{\widetilde{t}_{1}}$ & 1034.6 & 1528.3 & 1024.7 & 1514.8 & 1523.5 \\
\hline$m_{\widetilde{b}_{1}}$ & 1064.3 & 1568.6 & 1057.8 & 1552.1 & 1555.2 \\
\hline$m_{\widetilde{l}_{L}}$ & 380.3 & 627.3 & 617.5 & 617.9 & 618.7 \\
\hline$m_{\widetilde{l}_{R}}$ & 364.5 & 611.8 & 606.7 & 608.5 & 610.6 \\
\hline$m_{\widetilde{\nu}_{L}}$ & 372.4 & 624.5 & 611.7 & 612.9 & 613.5 \\
\hline$m_{\widetilde{\nu}_{R}}$ & 141.4 & 412.2 & 264.1 & 264.6 & 331.7 \\
\hline$m_{h}$ & 124.6 & 124.1 & 126.1 & 124.5 & 124.7 \\
\hline$\Delta m_{C P}(\mathrm{MeV})$ & 0.004 & 25.7 & 900 & 0.004 & 0.003 \\
\hline$\Delta m_{1}$ & 1.9 & 2.5 & 4.0 & -0.1 & -0.1 \\
\hline$\Delta m_{2}$ & 2.2 & 3.8 & 4.0 & 0.4 & 0.2 \\
\hline$\Delta M$ & 162.2 & 96.2 & 43 & 40.9 & 75.5 \\
\hline$\Omega h^{2}$ & & 0.11 & & & \\
\hline$\sigma_{S I}(\mathrm{pb})$ & & $1.4 \times 10^{-10}$ & & & \\
\hline $\sin \theta^{j}\left(\times 10^{-2}\right)$ & 0.002 & 10.9 & 0.046 & 0.224 & 0.004 \\
\hline$B R\left(\widetilde{\chi}_{1}^{ \pm} \rightarrow l \widetilde{\nu}\right)$ & 0.13 & 1.00 & 0.34 & 1.0 & 1.0 \\
\hline$B R\left(\widetilde{\chi}_{2}^{0} \rightarrow W^{\mp *} \widetilde{\chi}_{1}^{ \pm}\right)$ & 0.12 & 0.0 & 0.09 & 0.0001 & 0.10 \\
\hline$B R\left(\widetilde{\chi}_{2}^{0} \rightarrow W^{\mp *} \widetilde{\chi}_{1}^{ \pm} \rightarrow l \widetilde{\nu} W^{* \mp}\right)$ & 0.015 & 0.0 & 0.031 & 0.0001 & 0.10 \\
\hline
\end{tabular}

Table 4. Low energy input parameters and sparticle masses for the benchmarks used in the current study. All soft mass parameters and mass differences are in GeV. Mass differences amongst the different higgsino sector sparticles, $\Delta m_{1}$ and $\Delta m_{2}$, are as defined in section 2.2. Additionally, $\Delta M=m_{\widetilde{\chi}_{1}^{ \pm}}-m_{\widetilde{\nu}}$ represents the mass gap between the chargino and the sneutrino LSP and $\theta^{j}$ represents the mixing angle between the lightest left and right sneutrinos. 
there is a large mass gap between the higgsinos and the LSP, $\Delta M\left(=m_{\widetilde{\chi}_{1}^{ \pm}}-m_{\widetilde{\nu}_{\mathrm{LSP}}}\right) \sim$ $162 \mathrm{GeV}$. The first two generation sleptons are of masses $\sim 360 \mathrm{GeV}$ to facilitate left-right mixing in the sneutrino sector. The mixing in the left-right sneutrino is $\mathcal{O}\left(10^{-5}\right)$, such that for BP1 the three body decay of $\widetilde{\chi}_{1}^{ \pm}$, i.e. $\operatorname{BR}\left(\widetilde{\chi}_{1}^{ \pm} \rightarrow \widetilde{\chi}_{1}^{0} W^{ \pm *}\right)$ dominates $(\sim 88 \%)$ over the two-body decay, $\operatorname{BR}\left(\widetilde{\chi}_{1}^{ \pm} \rightarrow l \widetilde{\nu}\right)(\sim 12 \%)$. For a heavier slepton mass, a larger $T_{\nu}$ value is required for a similar left-right mixing angle and vice versa. Thus, we can fix the leptonic branching of the chargino either by lowering the left slepton mass or increasing $T_{\nu}$, and hence the left-right mixing in the sneutrino sector. Since the softer decay products from the three body decay produced from the off-shell $W$ pass undetected owing to the compression in the electroweakino sector, the two body leptonic decay is of interest, although subdominant. $\widetilde{\chi}_{1}^{0}$ and $\widetilde{\chi}_{2}^{0}$ dominantly decay to a $\nu \widetilde{\nu}$ pair contributing to missing energy signal. The dominant signals to look for in this case are mono-lepton $+\mathbb{E}_{T}$ and to a lesser extent opposite-sign and same-sign di-lepton events owing to the small leptonic branching of chargino.

Further, we choose a benchmark BP2-a, consistent with current data and similar to BP1, but with an increased left-right mixing in the sneutrino sector and satisfying thermal relic density in presence of heavy higgs resonance, of mass $\sim 824 \mathrm{GeV}$. It represents a point in the $M_{1} M_{2}>0$ and $\mu<0$ plane with $M_{1}=1.5 \mathrm{TeV}$ and $M_{2}=1.8 \mathrm{TeV}$ with the chargino decaying completely to the lepton and sneutrino mode. Here $\Delta m_{1}=2.5 \mathrm{GeV}, \Delta m_{2}=$ $3.8 \mathrm{GeV}$. We focus only on signals from the electroweakino sector and choose to keep the first and second generation left and right sleptons $\sim 600 \mathrm{GeV}$ such that their production cross-sections are negligible at $13 \mathrm{TeV}$ LHC, thus reducing any additional contributions to the leptonic final states. Owing to the large left-right mixing in the sneutrino sector, the higgsinos decay entirely to the sneutrino final state. Thus, the dominant signals from this scenario are monolepton and opposite sign dileptons along with missing energy.

We choose another spectrum BP2-b similar to BP2-a but for a lighter $\mu=300 \mathrm{GeV}$ and LSP mass $\sim 264 \mathrm{GeV}$ such that $\Delta M \sim 40 \mathrm{GeV}$. Thus, we choose a nearly compressed spectrum BP2-b where the leptons are much softer as compared to those of BP2-a in order to study the prospects of such a spectrum in presence of a $\widetilde{\nu}$ LSP. The dominant signals to look for are mono-lepton, opposite-sign di-lepton and same-sign di-lepton along with missing transverse energy.

BP3 and BP4 represent spectra with $M_{1} M_{2}<0$ and $\mu>0$ with $M_{1}=$ $-860(-1150) \mathrm{GeV}, M_{2}=2.5 \mathrm{TeV}$ and $\tan \beta=5$ such that the NLSP is the $\widetilde{\chi}_{1}^{ \pm}$. For BP3 we also choose a large left-right sneutrino mixing $\left(\mathcal{O}\left(10^{-3}\right)\right.$ while the LSP mass is $264 \mathrm{GeV}$. This leads to a tightly compressed electroweakino sector with $\widetilde{\chi}_{1}^{ \pm}$as the NLSP as discussed in section 2.2. Hence the only allowed decay of the chargino is the two body leptonic decay to the LSP with $\operatorname{BR}\left(\widetilde{\chi}_{1}^{ \pm} \rightarrow l \widetilde{\nu}\right)=100 \%$. Thus this region of parameter space favors the di-lepton channel with missing energy from chargino pair production. However the di-lepton channel suffers from a huge SM background and is much difficult to observe. Again the larger cross section for chargino-neutralino production only contributes to the mono-lepton channel as the decay of the heavier neutralinos to the chargino is rather suppressed for BP3 in order to respect the bounds from existing same-sign di-lepton searches. Hence for this particular benchmark the dominant signal to look for is mono-lepton $+\mathbb{E}_{T}$ 


\begin{tabular}{|c|c|c|c|c|c|c|}
\cline { 3 - 7 } \multicolumn{2}{c|}{} & \multicolumn{5}{c|}{ Luminosity $\left(\right.$ in $f b^{-1}$ ) for $3 \sigma$ excess } \\
\hline Analyses & Reference & BP1 & BP2-a & BP2-b & BP3 & BP4 \\
\hline$l^{ \pm} l^{\mp}(\mathrm{SF})+0$ jet $+\mathbb{E}_{T}$ & {$[132]$} & 13397 & 812 & - & - & 958 \\
$l^{ \pm} l^{\mp}(\mathrm{DF})+0$ jet $+\mathbb{E}_{T}$ & {$[132]$} & 2191 & 162 & - & - & 104 \\
$l^{ \pm} l^{\mp}+0$ jet $+\mathbb{E}_{T}$ & {$[165]$} & - & 2223 & - & - & 385 \\
\hline$l^{ \pm} l^{ \pm}+0$ jet $+\not_{T}$ & {$[167]$} & - & - & 1997 & - & 2726 \\
$l^{ \pm} l^{ \pm}+1$ jet $+\mathbb{E}_{T}$ & {$[167]$} & - & - & 4039 & - & 4901 \\
\hline
\end{tabular}

Table 5. Forecast for luminosity for $3 \sigma$ excess using present experimental searches using $36 \mathrm{fb}^{-1}$ of data at LHC. The blank spaces indicate that the benchmark is not sensitive to the final state analysis. We do not show the forecast from current monoleptonic searches as it gives much weaker sensitivity to our scenario.

and, to a lesser extent, opposite sign di-lepton $+\mathbb{E}_{T}$. However, other choices of benchmark points in this region of parameter space would allow same-sign di-lepton signal along with missing energy making it very interesting and clean mode for discovery. This can be the preferred channel for much larger $\mu$. We demonstrate a single benchmark, BP4, with $\mu=400 \mathrm{GeV}$ for this purpose.

Note that BP2-a is the only benchmark shown with the correct relic density $\left(\Omega h^{2}=\right.$ 0.11) suggesting that the LSP in this case is a thermal DM candidate. While the other benchmarks are assumed to have non-thermal DM we could have made them thermal by adjusting the mass of one of the heavy Higgs to achieve resonant annihilations and satisfy the relic density criterion. However, from the collider point of view the relic value will not affect the signals at LHC for any of the benchmark points and in the process neither differentiate a thermal relic from a non-thermal one.

Before we propose our analysis for observing the signal at LHC we use the existing analyses and forecast the integrated luminosity that would be required to observe a $3 \sigma$ excess at LHC for each of the benchmark points. We summarize our observations in table 5. For the above estimates, we have used the SM background events from the given references for respective analyses as shown in the table while we have computed the signal events in Madanalysis-v5.

\subsection{Collider analyses}

Simulation set-up and analyses. Our focus in this study is on leptonic channels with up to one ISR jet $\left(p_{T}>40 \mathrm{GeV}\right)$. We consider no extra partons at the matrix element level while generating the parton-level events for the signal using MadGraph-v5 [177-179]. Following the event generation at parton-level, showering and hadronisation of the events are performed using Pythia-v8 [180, 181]. Subsequently detector simulation is performed using Delphes-v3 [182-184]. Default dynamic factorization and renormalization scales of MadGraph-v5 have been used with CTEQ6L [185] as the parton distribution functions (PDF). Jets are reconstructed using Fastjet [186] with a minimum $p_{T}$ of $20 \mathrm{GeV}$ in a cone of $\Delta R=0.4$ using the anti- $k_{t}$ algorithm [187]. The charged leptons $(e, \mu)$ are reconstructed 
in a cone of $\Delta R=0.2$ with the maximum amount of energy deposit from other objects in the cone limited to $10 \%$ of the $p_{T}$ of the lepton. Photons are also reconstructed similar to the leptons in a cone of $\Delta R=0.2$, with the maximum energy deposit from other objects in the cone being at most $10 \%$ of the $p_{T}$ of the photon.

SM backgrounds have also been generated using MadGraph-v5, Pythia-v6 [179] and visible objects reconstructed at the detector level using Delphes-v3 [182-184]. Dominant SM backgrounds such as $l \nu+0,1 j$ and Drell Yan $\left(l^{+} l^{-}+0,1 \mathrm{j}\right)$ with large production cross-sections have been generated upto 1 extra parton. The matching between shower jets and jets produced at parton level is done using MLM matching with showerKT algorithm using $p_{T}$ ordered showers and a matching scale QCUT $=20 \mathrm{GeV}$. Signal and background analysis has been performed using MadAnalysis-v5 [188-190].

Primary selection criteria. We choose the following basic criteria for leptons (only $e^{ \pm}$ and $\left.\mu^{ \pm}\right)$, jets and photons for both signal and background:

- We select leptons $(\mathrm{e}, \mu)$ satisfying $p_{T}>10 \mathrm{GeV}$ and $|\eta|<2.5$.

- We choose photons with $p_{T}>10 \mathrm{GeV}$ and $|\eta|<2.5$.

- Reconstructed jets are identified as signal jets if they have $p_{T}>40 \mathrm{GeV}$ and $|\eta|<2.5$.

- Reconstructed b-tagged jets are identified with $p_{T}>40 \mathrm{GeV}$ and $|\eta|<2.5$.

- Jets and leptons are isolated such that $\Delta R_{l j}>0.4$ and $\Delta R_{l l}>0.2$.

\subsection{Mono-lepton $+\leq 1$ jet $+\boldsymbol{E}_{T}$ signal}

The presence of a sneutrino LSP opens up decay channels of the lightest chargino (neutralino) to a lepton (neutrino) and sneutrino. In such cases, mono-lepton signals with missing energy and few jets (mainly from ISR) arise dominantly from $\widetilde{\chi}_{1}^{ \pm} \widetilde{\chi}_{1}^{0}, \widetilde{\chi}_{1}^{ \pm} \widetilde{\chi}_{2}^{0}$ with $\tilde{\chi}_{1}^{ \pm} \rightarrow l \widetilde{\nu}$ and $\widetilde{\chi}_{1 / 2}^{0} \rightarrow \nu \widetilde{\nu}$. Sub-dominant contributions to the signal may also arise from $\tilde{\chi}_{1}^{ \pm} \widetilde{\chi}_{1}^{ \pm}$pair production when one of the chargino decays to a soft lepton (via the three body decay to the neutralino) and the other one decays to a hard lepton and the LSP. Smaller contributions to the signal also come from $\widetilde{\chi}_{1}^{0} \widetilde{\chi}_{2}^{0}$ production with $\widetilde{\chi}_{2}^{0}$ decaying to a chargino and soft decay products while $\widetilde{\chi}_{1}^{0}$ decays invisibly or vice versa if the chargino is the lightest among the higgsinos.

Dominant background to this signal come from SM processes:

- $l^{ \pm} \nu+0,1$ jets (including contributions from both on-shell and off-shell $W$ boson),

- $t \bar{t}$ (where one of the top quark decays hadronically and the other semi-leptonically).

- Single top quark production $(t(\bar{t}) j, t W)$.

- $W^{+} W^{-}+$jets $(W \rightarrow l \nu, W \rightarrow j j)$.

- $t \bar{t} W+$ jets (when both top quarks decay hadronically and $W \rightarrow l \nu$ ) and

- $W Z$ (with $W \rightarrow l \nu, Z \rightarrow \nu \bar{\nu} / j j)$. 


\begin{tabular}{|c|c|c|c|c|c|c|}
\hline \multirow{2}{*}{ Signal } & \multicolumn{7}{|c|}{ Number of events after cut } \\
\cline { 2 - 7 } & Preselection(M1) & M2 & M3 & M4 & M5 & M6 \\
\hline BP1 & 2543 & 1987 & 1946 & 1936 & 1601 & 1429 \\
BP2-a & 1495 & 944 & 922 & 916 & 706 & 611 \\
BP2-b & 6252 & 3194 & 3128 & 3118 & 2462 & 2215 \\
BP3 & $1.06 \times 10^{4}$ & 1664 & 1614 & 1601 & 1138 & 919 \\
BP4 & 3919 & 1793 & 1751 & 1740 & 1258 & 1074 \\
\hline
\end{tabular}

Table 6. Mono-lepton + missing energy signal final state number of events at $100 \mathrm{fb}^{-1}$ for SUSY signals. Note that the events have been rounded-off to the nearest integer. Cross-sections have been scaled using NLO K-factors obtained from Prospino.

Other subdominant contributions come from $t \bar{t}$ (where both top quarks decaying semileptonically), Drell Yan process $\left(l^{+} l^{-}+0,1 j\right)$ and $Z Z,\left(Z \rightarrow l^{+} l^{-}, Z \rightarrow \nu \bar{\nu} / j j\right)$ from misidentification of one of the leptons fail to meet the isolation cuts required to identify signal leptons or even hadronic energy mismeasurements leading to jets faking leptons. Smaller contributions may also arise from triple gauge boson production with one of the gauge boson decaying leptonically and the others hadronically. However, these are negligible compared to the $l \nu+j$ jets contribution. Other indirect contributions may arise from energy mismeasurements of jets as missing energy.

In order to select one lepton + missing energy signal, we implement the following criteria for both signal and backgrounds:

- M1: the final state consists of a single lepton with $p_{T}>25 \mathrm{GeV}$ and no photons.

- M2: since the dominant background contributions arise from W bosons, a large cut on the transverse mass, $M_{T}\left(l, \mathbb{E}_{T}\right)>150 \mathrm{GeV}$, where

$$
M_{T}\left(l, \notin_{T}\right)=\sqrt{2 p_{T}(l) \notin_{T}(1-\cos (\Delta \phi))} .
$$

$\Delta \phi$ denotes the azimuthal angle separation between the charged lepton $\overrightarrow{p_{T}}$ and $\overrightarrow{\mathbb{E}_{T}}$. A large cut on $M_{T}$ reduces SM background contributions from $l \nu+0,1$ jet, $W Z$, $W W$ and $t \bar{t}$ substantially as compared to the signal as seen in cut flow table 6 and 7 as well as figure 11.

- M3: events with atleast one b-tagged jet with $p_{T}>40 \mathrm{GeV}$ are rejected in order to reduce contribution from channels involving top quarks while leaving SUSY signals mostly unaffected.

- M4: as seen from figure 10 the weakly produced SUSY signals have a comparatively lower jet multiplicity compared to SM background processes involving strong production such as $t \bar{t}$ or single top. Thus a cut on the jet multiplicity in the signal events help to suppress the large SM background from these sources. Thus, we demand jet multiplicity, $N_{\text {jet }} \leq 3$. 

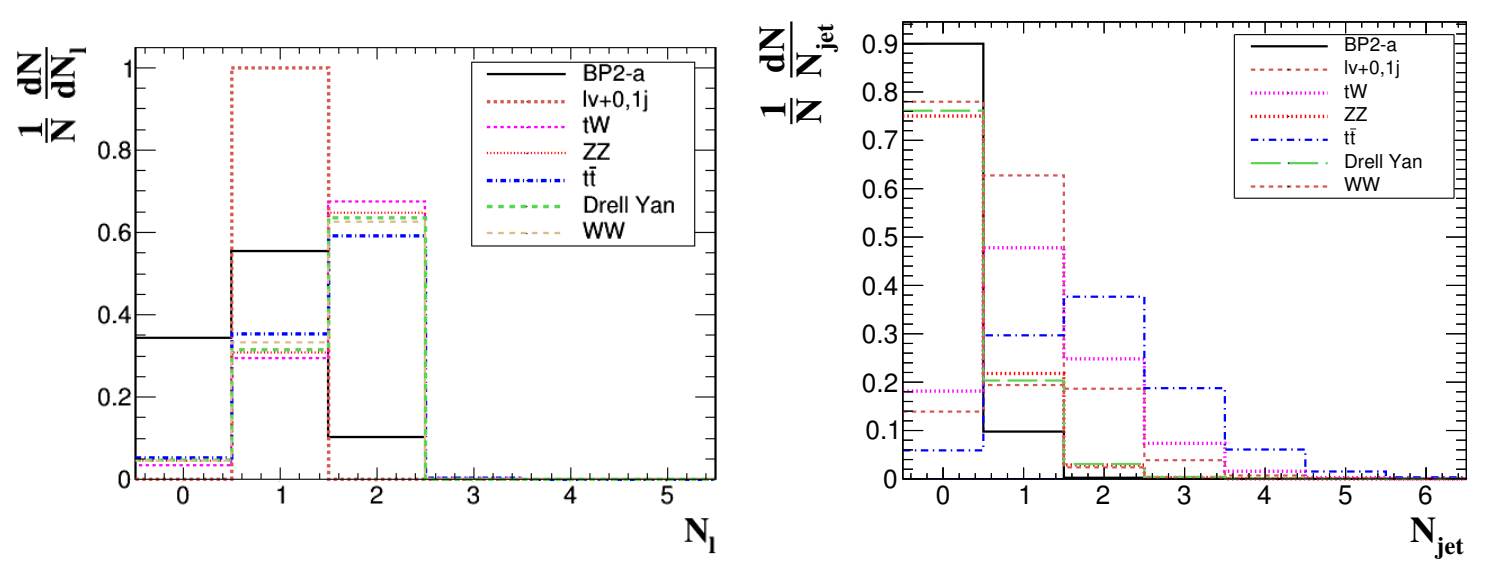

Figure 10. Normalized distributions for lepton and jet multiplicity for benchmark BP2 and dominant SM backgrounds channels. respectively.

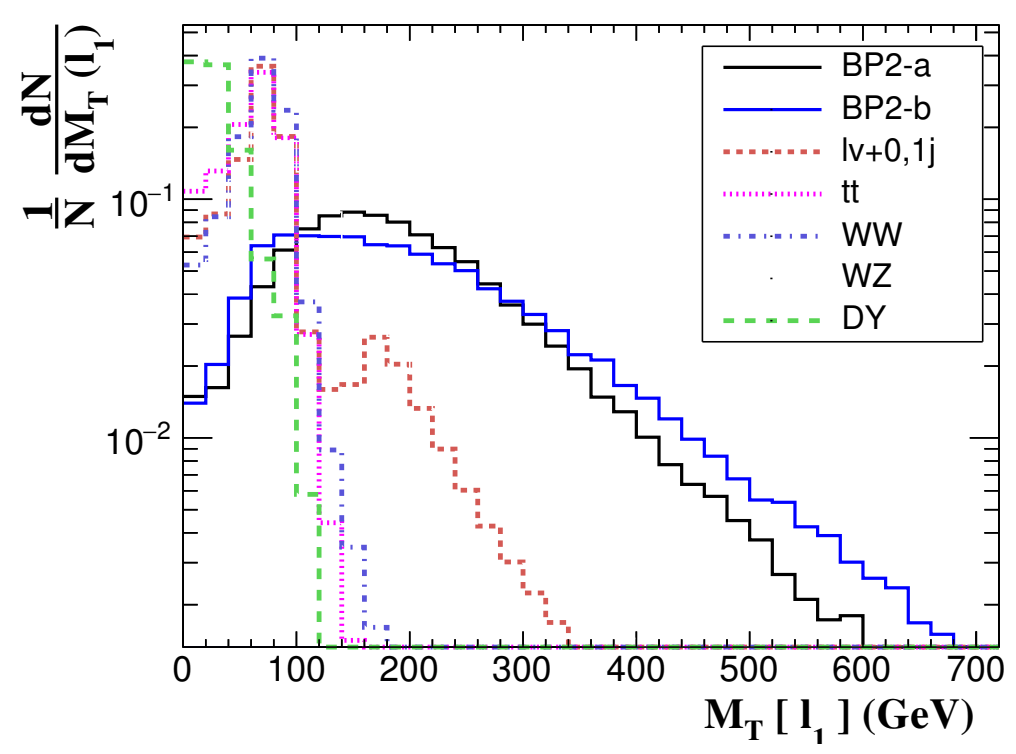

Figure 11. Normalized distribution for $M_{T}\left(l_{1}\right)$, the transverse mass of the leading lepton for SUSY signal BP2-a and BP2-b against the dominant SM backgrounds after preselection cut M1.

- M5: since SUSY signals have a large missing energy compared to the SM background, $\mathscr{E}_{T}>100 \mathrm{GeV}$ helps to reduce contributions from background.

- M6: in addition the events are made quiet from hadronic activity by demanding at most 1 jet in the final state. This helps to further reduce backgrounds events from $t \bar{t}$ and single top production.

In table 6 and 7 we list the number of events observable at $13 \mathrm{TeV}$ LHC at $100 \mathrm{fb}^{-1}$, for the signal and SM background respectively. Although most of the SM background events could be suppressed, the continuum background from $l \nu+0,1 j$ survives most of the cuts. The required luminosities for observing a $3 \sigma$ and $5 \sigma$ excess for the mono-lepton 


\begin{tabular}{|c|c|c|c|c|c|c|}
\hline SM & \multicolumn{7}{|c|}{ Number of events after cut } \\
\cline { 2 - 7 } Backgrounds & Preselection(M1) & M2 & M3 & M4 & M5 & M6 \\
\hline$l \nu+0,1 j$ & $1.07 \times 10^{7}$ & $1.09 \times 10^{6}$ & $1.08 \times 10^{6}$ & $1.08 \times 10^{5}$ & $5.77 \times 10^{5}$ & $5.49 \times 10^{5}$ \\
Drell Yan & $3.52 \times 10^{7}$ & $3.47 \times 10^{4}$ & $3.34 \times 10^{4}$ & 5991 & 5272 & 3674 \\
$W W$ & $8.04 \times 10^{5}$ & 5696 & 5485 & 5446 & 1329 & 1130 \\
$W Z$ & $1.56 \times 10^{5}$ & $2.54 \times 10^{4}$ & $2.48 \times 10^{4}$ & $2.20 \times 10^{4}$ & $1.55 \times 10^{4}$ & 11523 \\
$Z Z$ & 4938 & 912 & 900 & 899 & 551 & 492 \\
$t \bar{t}$ & $2.04 \times 10^{6}$ & $5.97 \times 10^{4}$ & $1.79 \times 10^{4}$ & $1.68 \times 10^{4}$ & $1.17 \times 10^{4}$ & 6399 \\
Single top & $3.68 \times 10^{6}$ & $2.05 \times 10^{4}$ & 8517 & 8088 & 2659 & 1603 \\
\hline Total & \multicolumn{7}{|l}{} & $5.74 \times 10^{5}$ \\
\hline
\end{tabular}

Table 7. Mono-lepton + missing energy signal final state number of events at $100 \mathrm{fb}^{-1}$ for $\mathrm{SM}$ background. Note that the events have been rounded-off to the nearest integer. Cross-sections scaled with K-factors at NLO [178] and wherever available, NNLO [191-196] have been used.

\begin{tabular}{|c|c|c|}
\hline Signal & $\mathcal{L}_{3 \sigma}\left(\mathrm{fb}^{-1}\right)$ & $\mathcal{L}_{5 \sigma}\left(\mathrm{fb}^{-1}\right)$ \\
\hline BP1 & 254 & 704 \\
BP2-a & 1384 & 3485 \\
BP2-b & 106 & 293 \\
BP3 & 613 & 1701 \\
BP4 & 448 & 1245 \\
\hline
\end{tabular}

Table 8. Required luminosities for discovery of mono lepton final states with missing energy at $\sqrt{s}=13 \mathrm{TeV}$ LHC.

$+\mathbb{E}_{T}$ channel are given in table 8 . The statistical significance is computed using:

$$
\mathcal{S}=\sqrt{2\left[(s+b) \ln \left(1+\frac{s}{b}\right)-s\right]}
$$

where $s$ and $b$ refer to the number of signal and background events after implementing the cuts M1-M6 respectively.

We find that the best signal significance is obtained by retaining at least one jet in the signal for all the benchmarks since the dominant background $l \nu+0,1 j$ and signal both have only ISR jet contributions. We note that requiring large $M_{T}, \mathbb{E}_{T}$ and one jet in the final state helps to improve the signal significance. Among all the benchmarks, BP2-a and BP4 have highest leptonic branching fraction for the chargino (100\%) as well as a large mass gap $\Delta M$ between the chargino and LSP. This leads to a relatively high cut efficiency for the signal. However since BP2-a corresponds to $|\mu|=500 \mathrm{GeV}$, the overall required luminosity for $3 \sigma$ excess is $\sim 1400 \mathrm{fb}^{-1}$. For BP4 with $\mu=300 \mathrm{GeV}$ and thus a higher production cross-section, the required luminosity is $\sim 500 \mathrm{fb}^{-1}$. BP1, having a large $\Delta M$ but lower chargino leptonic branching fraction, i.e., $\sim 12 \%$ would require $254 \mathrm{fb}^{-1}$ of data for observing a $3 \sigma$ excess at LHC. The relatively compressed spectra BP2-b and BP3 although with large leptonic branching fractions of the chargino, i.e. $\sim 37 \%$ and $100 \%$ respectively, have a lower cut efficiency owing to a smaller $\Delta M \sim 40 \mathrm{GeV}$. Thus the 
corresponding leptons would be soft compared to BP1 and BP2-a. Therefore BP2-b and BP3 require higher luminosity $106 \mathrm{fb}^{-1}$ and $613 \mathrm{fb}^{-1}$ respectively for observation.

\subsection{Di-lepton +0 jet $+\boldsymbol{E}_{T}$ signal}

The challenge in having a multi-lepton signal from the production of compressed higgsinolike electroweakinos comes from the fact that the decay products usually lead to soft final states. However, with a sneutrino LSP and the possibility of the decay of the chargino to a hard lepton and the LSP leads to a healthy di-lepton signal with large missing energy (from $\widetilde{\chi}_{1}^{+} \tilde{\chi}_{1}^{-}$as well as $\widetilde{\chi}_{1}^{ \pm} \widetilde{\chi}_{2}^{0}$ pair production, provided the next-to-lightest neutralino decay yields a lepton via the chargino). A sub-dominant contribution also arises from $\widetilde{\chi}_{1}^{0} \tilde{\chi}_{2}^{0}$ with each of the neutralino decaying to a chargino and an off-shell $W$ boson which gives soft decay products. The chargino then decays to a charged lepton and sneutrino LSP. This happens most favorably when chargino is the lightest of the higgsinos. Owing to the Majorana nature of $\widetilde{\chi}_{i}^{0}$ we can have signals for opposite-sign and same-sign di-lepton final states with large missing transverse energy. Hence we look into both the possibilities:

- Opposite sign di-lepton +0 jet $+\mathbb{E}_{T}$

- Same sign di-lepton +0 jet $+\mathbb{E}_{T}$

\subsubsection{Opposite sign di-lepton +0 jet $+\not_{T}$ signal}

Opposite sign di-lepton signal arises mainly from $\tilde{\chi}_{1}^{+} \widetilde{\chi}_{1}^{-}$production process. Sub-dominant contributions arise from $\widetilde{\chi}_{1}^{ \pm} \widetilde{\chi}_{1}^{0}, \widetilde{\chi}_{1}^{ \pm} \widetilde{\chi}_{2}^{0}$ and $\widetilde{\chi}_{1}^{0} \widetilde{\chi}_{2}^{0}$ as discussed before. The dominant SM contributions to the opposite sign di-lepton signal with missing energy come from $t \bar{t}, t W$ and Drell-Yan production. Among the di-boson processes, $W^{+} W^{-}\left(W^{+} \rightarrow l^{+} \nu, W^{-} \rightarrow\right.$ $\left.l^{-} \bar{\nu}\right), Z Z\left(Z \rightarrow l^{+} l^{-}, Z \rightarrow j j / \nu \bar{\nu}\right)$ and $W Z+$ jets $\left(W \rightarrow j j, Z \rightarrow l^{+} l^{-}\right)$also contribute substantially to the opposite sign di-lepton channel. The triple gauge boson processes may also contribute. However, these have a small production cross-section and are expected to be subdominant. There could also be fake contributions to missing energy from hadronic energy mismeasurements.

In figure 12 we show the normalized distributions for important kinematic variables for two benchmarks BP2-a and BP2-b with $\Delta M=100,40 \mathrm{GeV}$ respectively along with the dominant SM backgrounds after selecting the opposite sign-di-lepton state (D1). We find that as expected the lepton $p_{T}$ distribution for $\mathbf{B P 2 - a}$ is much harder than the SM backgrounds processes whereas for BP2-b with a lower mass gap between the chargino and LSP, the leptons are much softer and the distributions have substantial overlap with the backgrounds. We further use the other kinematic variables,

$$
\mathbb{E}_{T}=\left|\Sigma_{i} \vec{p}_{T_{i}}\right| \text { and } M_{l^{+} l^{-}}^{2}=\left(p_{l_{1}}+p_{l_{2}}\right)^{2}
$$

(where $i$ runs over all visible particles in the final state) represent the transverse missing energy and invariant mass-squared of the di-lepton final state respectively which peak at higher values for SUSY signals over backgrounds in BP2-a whereas BP2-b still retains a large overlap with the SM backgrounds. However, the largest source of background for the 
di-lepton background coming from Drell-Yan process can be removed safely by excluding the $Z$ boson mass window for $M_{l^{+} l^{-}}$. Since the SUSY signals do not arise from a resonance the exclusion of the $Z$ mass window is expected to have very little effect on the signal events. We further note that removing b-tagged jets would also be helpful in removing SM background contributions from the strongly produced top quark channels which have huge cross sections at the LHC.

Another kinematic variable of interest to discriminate between SUSY signals and SM backgrounds is the $M_{T_{2}}$ variable [197] constructed using the leading and sub-leading lepton $\overrightarrow{p_{T}}$ and $\overrightarrow{E_{T}}$. For processes with genuine source of $\mathbb{E}_{T}$ there is a kinematic end point of $M_{T_{2}}$ which terminates near the mass of the parent particle producing the leptons and the invisible particle. In SM, channels such as $t \bar{t}, t W, W^{+} W^{-}$involving a $W$ boson finally giving the massless invisible neutrino in the event, the end-point would be around $80 \mathrm{GeV}$. For SUSY events the invisible particle is not massless and therefore the visible lepton $p_{T}$ will depend on the mass difference. Thus the end-point in the signal distribution would not have a cut-off at the parent particle mass anymore. For BP2-a which has a large $\Delta M$ the end point is expected at larger values $(\sim 200) \mathrm{GeV}$. However for BP2-b, where the available phase space is small for the charged lepton due to smaller $\Delta M$ the $M_{T_{2}}$ distribution is not very wide and has an end-point at a much lower value. Thus a strong cut on this variable is not favorable when the sneutrino LSP mass lies close to the electroweakino's mass.

Following the features of the kinematic distributions, we implement the following optimal selection criteria as follows for both signal and backgrounds:

- D1: the final state consists of two opposite sign leptons and no photons.

- D2: the leading lepton has $p_{T}>20 \mathrm{GeV}$ and the sub-leading lepton has $p_{T}>10 \mathrm{GeV}$.

- D3: $M_{l^{+} l^{-}}>10 \mathrm{GeV}$ helps remove contributions from photon mediated processes while the $Z$ mass window is also removed by demanding that the opposite-sign same flavor di-lepton invariant mass satisfies $76<M_{l^{+} l^{-}}<106 \mathrm{GeV}$. This helps to reduce a large resonant contribution form the $Z$ exchange in Drell-Yan process.

- D4: we reject any $b$-jet by putting a $b$-jet veto (for $p_{T}>40 \mathrm{GeV}$ ). This helps in suppressing background events coming from top quark production.

- D5: we demand a completely hadronically quiet event by choosing zero jet multiplicity $\left(N_{\text {jet }}=0\right)$ in the signal events. This is effective in suppressing contributions from background processes produced via strong interactions.

- D6: we demand $\mathbb{E}_{T}>80 \mathrm{GeV}$ to suppress the large Drell-Yan contribution.

- D7: we demand $M_{T 2}>90 \mathrm{GeV}$ which helps reduce a majority of the other SM backgrounds.

- D8: $\mathbb{E}_{T}>100 \mathrm{GeV}$ is implemented to further reduce the SM backgrounds.

In table 9 we show the signal events that survive the above listed kinematic selections (cut-flow). We find that among all benchmarks, BP2-a is the most robust followed by 

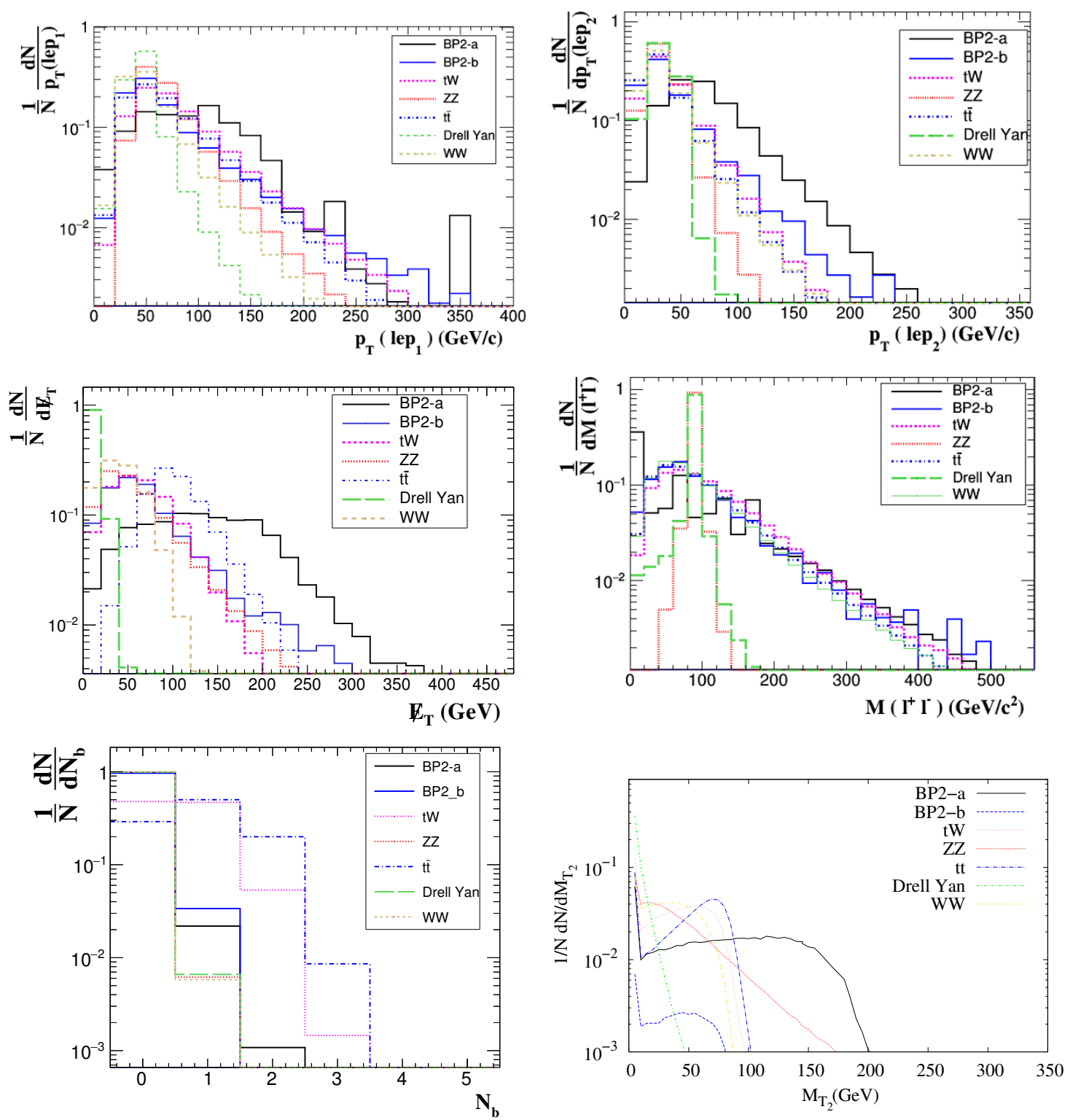

Figure 12. Normalized distributions of several kinematic variables after cut D1.

BP4. Note that we avoid using the $M_{T 2}$ cut on the benchmarks where the mass splitting between the chargino and the sneutrino LSP is small as D7 cut makes the signal events negligible. As pointed out earlier, the end-point analysis in $M_{T 2}$ is not favorable for small $\Delta M$ as seen in the signal and background distributions in figure 12. Thus BP2-b and BP3 have cuts D1-D6. In table 10 we plot the SM background events after each kinematic cuts. Quite clearly up to cut D6 the SM background numbers are quite large, and then drastically reduce after the $M_{T 2}$ cut (D7) is imposed.

In table 11 we give the required integrated luminosities to achieve a $3 \sigma$ and $5 \sigma$ statistical significance for the signal events of the benchmark points. Just like for the mono-lepton 


\begin{tabular}{|c|c|c|c|c|c|c|c|}
\hline \multirow{2}{*}{ Signal } & \multicolumn{7}{|c|}{ Number of events after cut } \\
\cline { 2 - 8 } & Preselection (D1) & D2 & D3 & D4 & D5 & D7 & D8 \\
\hline BP1 & 130 & 129 & 112 & 109 & 68 & 22 & 21 \\
BP2-a & 306 & 271 & 265 & 161 & 108 & 76 & 72 \\
BP4 & 209 & 298 & 246 & 241 & 153 & 81 & 40 \\
\hline
\end{tabular}

\begin{tabular}{|c|c|c|c|c|c|c|c|}
\hline \multirow{2}{*}{ Signal } & \multicolumn{6}{|c|}{ Number of events after cut } \\
\cline { 2 - 8 } & Preselection (D1) & D2 & D3 & D4 & D5 & D6 & \\
\hline BP2-b & 455 & 452 & 351 & 345 & 230 & 45 & \\
BP3 & 2424 & 2394 & 1840 & 1805 & 1186 & 189 & \\
\hline
\end{tabular}

Table 9. Opposite Sign di-lepton $+\mathscr{E}_{T}$ final state number of events at $100 \mathrm{fb}^{-1}$ for SUSY signals. Note that the events have been rounded-off to the nearest integer. Cross-sections have been scaled using NLO K-factors obtained from Prospino.

\begin{tabular}{|c|c|c|c|c|c|c|c|c|}
\hline \multirow{2}{*}{ Signal } & \multicolumn{8}{|c|}{ Number of events after cut } \\
\cline { 2 - 10 } & Preselection (D1) & D2 & D3 & D4 & D5 & D6 & D7 & D8 \\
\hline Drell Yan & $1.16 \times 10^{8}$ & $1.14 \times 10^{8}$ & $8.89 \times 10^{6}$ & $8.80 \times 10^{6}$ & $6.89 \times 10^{6}$ & 506 & 293 & 53 \\
$W^{+} W^{-}$ & $1.44 \times 10^{5}$ & $1.43 \times 10^{5}$ & $1.13 \times 10^{5}$ & $1.13 \times 10^{5}$ & $1.00 \times 10^{5}$ & 5813 & 24 & 12 \\
$Z Z$ & $1.71 \times 10^{4}$ & $1.71 \times 10^{4}$ & 656 & 651 & 504 & 117 & 50 & 45 \\
$W Z$ & $6.0 \times 10^{4}$ & $6.0 \times 10^{4}$ & 5399 & 5208 & 1554 & 92 & 6 & 4 \\
$t \bar{t}$ & $6.19 \times 10^{5}$ & $6.16 \times 10^{5}$ & $4.96 \times 10^{5}$ & $1.48 \times 10^{5}$ & $3.5 \times 10^{4}$ & $2.63 \times 10^{4}$ & 132 & 106 \\
$t W$ & $1.77 \times 10^{5}$ & $1.74 \times 10^{5}$ & $1.40 \times 10^{5}$ & $6.76 \times 10^{4}$ & $2.99 \times 10^{4}$ & 8181 & 114 & 51 \\
\hline Total & \multicolumn{10}{|c|}{} & 41009 & & 271 \\
\hline
\end{tabular}

Table 10. Opposite Sign di-lepton $+\mathbb{E}_{T}$ final state number of events at $100 \mathrm{fb}^{-1}$ for Standard Model backgrounds. Note that the events have been rounded-off to the nearest integer. Crosssections scaled with K-factors at NLO [178] and wherever available, NNLO [191-196] have been used.

case, BP2-a requires the least integrated luminosity and is in fact gives a $3 \sigma$ significance for much lower luminosity compared to mono-lepton signal. However for the rest of the benchmarks mono-lepton channel is more favorable while the opposite-sign di-lepton can act as a complementary channel for BP1 with higher luminosity and BP3 with the very-high luminosity option of LHC. BP2-b type of spectrum for the model is strongly suppressed in the di-lepton channel. The signal rates can be attributed to the fact that the leptonic branching of the chargino is much larger for BP2-a $(\sim 100 \%)$ than BP1 $(\sim 12 \%)$ and hence the signal is much more suppressed for BP1 than in BP2-a. For BP4 where the NLSP is the chargino, the opposite sign dilepton signal is a robust channel for discovery.

Thus this channel is not a likely probe for benchmarks with a smaller phase space, like BP2-b and BP3 in which cases, as seen in the previous section, mono-lepton signals fare better over di-lepton signals. Whereas for spectra like $\mathbf{B P 2 - a}$ and $\mathbf{B P 4}$, with a large phase space available, opposite sign di-lepton signals are much more sensitive than mono-lepton signals. In contrast spectra like BP1 with a lower leptonic branching of the chargino, mono-lepton + missing energy signal is still a better channel to look for than opposite sign di-lepton channel. 


\begin{tabular}{|c|c|c|}
\hline Signal & $\mathcal{L}_{3 \sigma}\left(\mathrm{fb}^{-1}\right)$ & $\mathcal{L}_{5 \sigma}\left(\mathrm{fb}^{-1}\right)$ \\
\hline BP1 & 568 & 1576 \\
BP2-a & 51 & 142 \\
BP2-b & $1.83 \times 10^{4}$ & $5.07 \times 10^{4}$ \\
BP3 & 1035 & 2875 \\
BP4 & 160 & 444 \\
\hline
\end{tabular}

Table 11. Required luminosities for discovery of opposite sign di-lepton $+\mathbb{E}_{T}$ final states at $\sqrt{s}=13 \mathrm{TeV}$ LHC.

\subsubsection{Same sign di-leptons +0 jet $+\boldsymbol{E}_{T}$ signal}

A more interesting and unique new physics signal at LHC in the di-lepton channel is the same-sign di-lepton mode. The same-sign di-lepton in the absence of missing transverse energy is a clear signal for lepton number violation and forms the backbone for most studies of models with heavy right-handed Majorana neutrinos. Even with missing energy, the same-sign di-lepton is a difficult final state to find within the SM and therefore a signal with very little SM background. Thus finding signal events in this channel would give very clear hints of physics beyond the SM.

In our framework of SUSY model the same-sign di-lepton signal with missing energy and few jets come from the production modes $\widetilde{\chi}_{1}^{ \pm} \widetilde{\chi}_{2}^{0}$ and/or $\widetilde{\chi}_{1}^{ \pm} \widetilde{\chi}_{1}^{0}$ where the lepton number violating contribution comes from the decay of the Majorana-like neutralinos given by $\widetilde{\chi}_{2}^{0} \rightarrow W^{\mp *} \widetilde{\chi}_{1}^{ \pm}$with $\tilde{\chi}_{1}^{ \pm} \rightarrow l \widetilde{\nu}$. We note that same-sign di-lepton backgrounds are rare in SM, with some small contributions coming from processes such as $p p \rightarrow W Z, Z Z, W^{+} W^{+} / W^{-} W^{-}+$jets, $t \bar{t} W$ and $t \bar{t} Z$ as well as from triple gauge boson productions such as $W W W$ where with two of the $W$ bosons being of same sign and the other decaying hadronically. Other indirect backgrounds can arise from energy mismeasurements, i.e, when jets or photons or opposite sign leptons fake a same sign di-lepton signal. ${ }^{7}$

For our analysis we select the same-sign di-lepton events using optimal cuts for both signal and background using the following kinematic criteria:

- S1: the final state consists of two charged leptons with same-sign and the leading lepton in $p_{T}$ must satisfy $p_{T}>20 \mathrm{GeV}$ with the sub-leading lepton having $p_{T}>15 \mathrm{GeV}$. Additionally we ensure that there are no isolated photon and $b$-jets in the final state.

- S2: a minimal cut on the transverse mass constructed with the leading charged lepton $\left(l_{1}\right), M_{T}\left(l_{1}, \vec{E}_{T}\right)>100 \mathrm{GeV}$ is chosen to reject background contributions coming from $W$ boson.

- S3: to suppress background from $W^{ \pm} W^{ \pm} \mathrm{jj}$ as well as those from $t \bar{t} W$, $t \bar{t} Z$ with higher jet multiplicities than the SUSY signal, we keep events with only up to 2 jets.

\footnotetext{
${ }^{7}$ There may be additional contributions for same-sign di-lepton coming from non-prompt and conversions which we have not considered [129].
} 


\begin{tabular}{|c|c|c|c|c|c|}
\hline \multirow{2}{*}{ Signal } & \multicolumn{5}{|c|}{ Number of events after cut: } \\
\cline { 2 - 6 } & Preselection (S1) & S2 & S3 & S4 & S5 \\
\hline BP1 & 5 & 5 & 5 & 4 & 2 \\
BP2-a & 3 & 2.5 & 2.3 & 1.6 & 1 \\
BP2-b & 32 & 23 & 22 & 15 & 8 \\
BP3 & 2 & 0.5 & 0.5 & 0.2 & 0.1 \\
BP4 & 30 & 23 & 23 & 13 & 7 \\
\hline
\end{tabular}

Table 12. Same sign di-lepton $+\mathbb{E}_{T}$ final state number of events at $100 \mathrm{fb}^{-1}$ for SUSY signals. Note that the events have been rounded-off to the nearest integer where relevant. Cross-sections have been scaled using NLO K-factors obtained from Prospino.

\begin{tabular}{|c|c|c|c|c|c|}
\hline \multirow[t]{2}{*}{ SM Backgrounds } & \multicolumn{5}{|c|}{ Number of events after cut } \\
\hline & Preselection $(\mathbf{S 1})$ & S2 & S3 & S4 & S5 \\
\hline$W Z$ & 3856 & 1053 & 930 & 194 & 39 \\
\hline$Z Z$ & 94 & 6 & 5 & 0.5 & 0.2 \\
\hline$W W W$ & 60 & 29 & 21 & 7 & 0.6 \\
\hline$W^{+} W^{+} j j$ & 416 & 175 & 116 & 56 & 2 \\
\hline$W^{-} W^{-} j j$ & 188 & 82 & 57 & 18 & 0.5 \\
\hline$t W$ & 40 & 20 & 19 & 7 & 4 \\
\hline$t \bar{t} W$ & 128 & 60 & 30 & 13 & 1 \\
\hline$t \bar{t}$ & 90 & 65 & 50 & 28 & 8 \\
\hline Total background & & & & & 55 \\
\hline
\end{tabular}

Table 13. Same sign di-lepton $+\mathbb{E}_{T}$ final state number of events at $100 \mathrm{fb}^{-1}$ for SM background. Note that the events have been rounded-off to the nearest integer where relevant. Cross-sections scaled with K-factors at NLO [178] and wherever available, NNLO [191-196] have been used.

- S4: a large missing energy cut, $\mathbb{E}_{T}>100 \mathrm{GeV}$ is implemented to reduce SM backgrounds.

- S5: finally we choose the events to be completely hadronically quiet and demand zero jets in the event.

In tables 12 and 13 we show the signal and backgrounds events after each selection cuts are imposed. As the same-sign signal is strongly constrained by existing LHC data, our benchmarks have been chosen to comply with the existing limits. Thus we find that our benchmark choices do not seem too robust in terms of signal rates, especially BP3 and BP4 which has the chargino as the NLSP. It is therefore important to point out that BP1 and BP2-a like spectra is naturally not favored to give a same-sign di-lepton signal while BP3 and BP4 are the most probable to give the same-sign signal but have been chosen to suppress the signal to respect existing constraints (by choosing very small branching for the neutralinos to decay to chargino) for two different $\mu$ values. However the spectra as reflected by BP2-b and BP4 satisfying existing constraints do present us with a significant number of event rates when compared to the background after cuts. 


\begin{tabular}{|c|c|c|}
\hline Signal & $\mathcal{L}_{3 \sigma}\left(\mathrm{fb}^{-1}\right)$ & $\mathcal{L}_{5 \sigma}\left(\mathrm{fb}^{-1}\right)$ \\
\hline BP2-b & 811 & 2251 \\
BP4 & 1052 & 3845 \\
\hline
\end{tabular}

Table 14. Required luminosities for discovery of same sign di-lepton final states with missing energy at $\sqrt{s}=13 \mathrm{TeV}$ LHC.

From the above cuts, we find that a large $M_{T}\left(l_{1}\right)$ cut coupled with a large $\mathbb{E}_{T}$ and the requirement of jet veto removes a large fraction of the dominant $W Z$ background as well as other fake contributions coming from $t \bar{t}$. Other genuine contributions to this channel from $W^{ \pm} W^{ \pm} j j, t \bar{t} W$ and $W W W$ having a lower production cross-section and are efficiently suppressed by cuts on $\mathbb{E}_{T}, M_{T}$ and applying a jet veto. Amongst all benchmarks, the most sensitive to the same sign di-lepton analysis are BP2-b where $\operatorname{BR}\left(\widetilde{\chi}_{2}^{0} \rightarrow \widetilde{\chi}_{1}^{ \pm} W^{\mp} \rightarrow l \widetilde{\nu}\right) \sim 3.3 \%$ and BP4 with $\operatorname{BR}\left(\widetilde{\chi}_{2}^{0} \rightarrow \widetilde{\chi}_{1}^{ \pm} W^{\mp} \rightarrow l \widetilde{\nu}\right) \sim 10 \%$. Note that BP2-b, with a smaller $\Delta M$ gives soft leptons and is therefore slightly suppressed and requires larger integrated luminosity $\sim 810 \mathrm{fb}^{-1}$ of data as shown in table 14. Although BP4 has a larger branching fraction, it requires $1052 \mathrm{fb}^{-1}$ of data at LHC for observing a $3 \sigma$ excess owing to a higher $\mu$ value compared to BP2-b. Thus the same-sign di-lepton can be a complementary channel to observe for benchmarks of BP2-b and BP4. We must again point out here that for BP3-like spectra with $\widetilde{\chi}_{1}^{ \pm}$NLSP the same-sign di-lepton would be the most sensitive channel of discovery, for large $|\mu|$ and small $M_{2}$, where the neutralino decay to chargino NLSP becomes large (see figures 5 and 6) because of the small SM background. In such a case both $\widetilde{\chi}_{1}^{0}$ and $\widetilde{\chi}_{2}^{0}$ will decay to the NLSP along with soft jets or leptons. Thus both $\widetilde{\chi}_{1}^{ \pm} \widetilde{\chi}_{2}^{0}$ and $\widetilde{\chi}_{1}^{ \pm} \widetilde{\chi}_{1}^{0}$ production channels would have contributed to the signal leading to a two-fold increase of the number of signal events and would be more sensitive to detect a sneutrino LSP scenario.

We conclude that conventional channels such as mono-lepton or opposite sign di-lepton channels however with low hadronic activity, i.e, with at most 1 jet or no jet would be extremely useful channels to look for cases of a sneutrino LSP. Detecting same sign dilepton signals at higher luminosities would further serve as a strong confirmatory channel for a sneutrino LSP scenario over a $\widetilde{\chi}_{1}^{0}$ LSP scenario as in the MSSM from the compressed higgsino sector and can exclude large portions of the regions with $M_{1}<0$. Our analyses also shows better signal significance for a given integrated luminosity, when compared to the forecast shown in table 5. Note that our estimates do not include any systematic uncertainties that may be present and would be dependent on the specific analysis of event topologies. However it is worthwhile to ascertain how our results fare in presence of such systematic uncertainties. To highlight this we assume a conservative $10 \%$ systematic uncertainty in each case. We find that the required integrated luminosities follow a similar scaling and our results for the luminosity vary by atmost $10 \%$ in most cases.

Dependence on flavor of $\widetilde{\boldsymbol{\nu}}_{\boldsymbol{R}}$ LSP. LHC searches explore different search channels involving the flavor of the leptons owing to their high reconstruction efficiency at the detector, for instance, e+ $\mathbb{E}_{T}, \mu+\mathbb{E}_{T}[161,162]$, ee/ $\mu \mu / e \mu$ final states associated with $\mathbb{E}_{T}[130,132]$. As we have considered both first and second generation sneutrinos to be 
light in this study, we qualitatively analyze the prospects of the signals studied by tagging the flavor of the leptons as well as consequences of a single light generation of sneutrino LSP assuming the net leptonic branching to be the same in both cases. ${ }^{8}$ Hence, for a single light sneutrino LSP, the observed events in the mono-lepton and di-lepton signals contribute to only a single choice of lepton flavor and vanishes for the rest. We compare the signal and background in this case for the same luminosity as before and comment on the results obtained for our benchmarks.

For mono-lepton signals with degenerate sneutrino LSP (first two generations), say, we look at only an electron in the final state. This would lead to reduction of both signal and background in table 6 and 7 by half such that the significance falls by a factor of $\sqrt{2}$. If a single generation of right-sneutrino was light, say $\widetilde{\nu}_{e}$, then only the background would reduce by a factor $1 / 2$. Since the signal remains unchanged as the chargino now decays completely to an electron and the lightest sneutrino the signal significance increases by a factor of $\sqrt{2}$. Consequently no signal is observed for the other flavor lepton channel, in this case $\mu$, where although the background decreases by half, no signal events are present.

For the di-lepton signal there are three possible channels $e e, \mu \mu$ and $e \mu$ with net branching fraction of around $1 / 4,1 / 4$ and $1 / 2$ respectively. We consider first the oppositesign di-lepton channel. For $e \mu$ final states, only different flavor lepton backgrounds such as from $W W, t \bar{t}$ or $t W$ contribute with a $\mathrm{BR} \simeq 2 / 3$. However contributions from same flavor di-lepton sources such as involving $Z$ boson fall. The total background thus reduces to nearly $70 \%$. Since signal in this channel also reduces to half thereby the significance falls. For channels with same flavor (SF) leptons, i.e, ee/ $\mu \mu$, dominant SF contributions are from $Z$ boson whereas sub-dominant contributions from top quark production channel reduce. Although SM background reduces so does the signal statistics and hence the significance. However, in presence of a single generation of light sneutrino we find that the signal significance improves by a factor of about $\sqrt{2}$. Note that if the LSP is $\widetilde{\nu}_{e}$ then the chargino decays to an electron and the LSP. Therefore $e e+\mathbb{E}_{T}$ channel significance improves whereas $\mu \mu$ and $e \mu$ channels vanish. Similarly, for an $\widetilde{\nu}_{\mu}$ LSP, $\mu \mu+\mathbb{E}_{T}$ channels improve whereas the rest vanish. Similar conclusions may be drawn for same sign dilepton channel, where the dominant backgrounds are $W Z$ and $W^{ \pm} W^{ \pm}$, the significance is expected to improve only for a single light generation of sneutrinos.

Some comments on the prospect of $\tau$ flavor searches and other channels. In this context, we also explore the discovery prospects of a natural higgsino sector and a single light $\widetilde{\nu}_{\tau}$ as the LSP. LHC has looked at final states with tau leptons, decaying hadronically, in the context of electroweakino searches. The electroweakino mass limits considerably reduce for tau lepton searches owing to the reduced reconstruction efficiency of hadronically decaying $\tau$ leptons $(\sim 60 \%)$ [198] compared to that of the light leptons $(\mathrm{e}, \mu)$ $(\sim 95 \%)$. From searches with one or two hadronically decaying tau leptons associated with light leptons lead to stronger limits from $\widetilde{\chi}_{1}^{ \pm}, \widetilde{\chi}_{2}^{0}$ production on $m_{\widetilde{\chi}_{1}^{ \pm}}, m_{\widetilde{\chi}_{2}^{0}}>800 \mathrm{GeV}$ for

${ }^{8}$ This may not correspond to the same parameter point since the presence of the other decay modes of $\widetilde{\chi}_{1}^{ \pm}$affect the leptonic branching for the single light sneutrino LSP case. However, when $\widetilde{\chi}_{1}^{ \pm}$is the NLSP, the net leptonic branching is the same in both cases. 
a bino-like $m_{\widetilde{\chi}_{1}^{0}}<200 \mathrm{GeV}$ for stau mass midway between the $\widetilde{\chi}_{1}^{ \pm}$and $\widetilde{\chi}_{1}^{0}$. For stau closer to the $\widetilde{\chi}_{1}^{ \pm}$, the limit [129], $m_{\widetilde{\chi}_{1}^{ \pm}}, m_{\widetilde{\chi}_{2}^{0}} \sim 1000 \mathrm{GeV}$ for $m_{\widetilde{\chi}_{1}^{0}} \sim 200 \mathrm{GeV}$. Limits on electroweakino searches from three tau lepton searches exclude wino-like degenerate $m_{\widetilde{\chi}_{2}^{0}}, m_{\widetilde{\chi}_{1}^{ \pm}}>600 \mathrm{GeV}$ for a bino-like $m_{\widetilde{\chi}_{1}^{0}}<200 \mathrm{GeV}$ [129] for stau mass midway between the $\widetilde{\chi}_{1}^{ \pm}$and $\widetilde{\chi}_{1}^{0}$. Limits from opposite sign tau lepton searches [199] reinterpreted from $\widetilde{\chi}_{1}^{ \pm} \widetilde{\chi}_{2}^{0}$ production and decaying via intermediate sleptons lead to $m_{\widetilde{\chi}_{2}^{0}}, m_{\widetilde{\chi}_{1}^{ \pm}}>760 \mathrm{GeV}$ for $m_{\widetilde{\chi}_{1}^{0}}<200 \mathrm{GeV}$. From opposite sign di-tau searches reinterpreted in context of chargino pair production leads to a bound close to $650 \mathrm{GeV}$ on chargino for LSP masses up to $100 \mathrm{GeV}[199]$.

For the current scenario of a compressed electroweakino sector in presence of a light $\widetilde{\nu}_{\tau}$ LSP, the signals from the low-lying compressed higgsino sector would be:

- Mono- $\tau$ jet $+\mathbb{E}_{T}$

- Di $\tau$ jets $+\mathbb{E}_{T}$

For the mono-tau channel, both signal and background scale by the tau reconstruction efficiency, $\epsilon_{R}=0.6$ is the tau reconstruction efficiency. Further a factor of $\frac{1}{2}$ comes in for the background since the branching of $W$ or $Z$ boson to light leptons is roughly twice that to the tau lepton as for a $\widetilde{\nu}_{e / \mu}$ LSP. However, owing to the reduced tau reconstruction efficiency, the signal significance falls by $\sim \sqrt{\epsilon_{R}} \sim 0.78$. Similarly, for the di-tau channels, the significance scales by $\epsilon_{R} \sim 0.6$. Hence, the estimated reach of the higgsino mass parameter, $\mu$ is expected to weaken for a $\widetilde{\nu}_{\tau}$ LSP compared to $\widetilde{\nu}_{e / \mu}$ LSP.

Note that, the pionic decay modes of the $\tilde{\chi}_{1}^{ \pm}$and $\tilde{\chi}_{2}^{0}$ can dominate among the hadronic modes as the respective mass differences become less than about a $\mathrm{GeV}$. While we have used form factors to estimate the pionic branching fractions, we have not considered the possibility of late decay into pions in this work. This is because we have ensured that in the parameter space of our interest the two body mode to the lightest sneutrino(s) always remain prompt. Further, the potential of the loop-induced channel $\tilde{\chi}_{2}^{0} \rightarrow \tilde{\chi}_{1}^{0} \gamma$ in deciphering the scenario has not been explored in the present work. While the photons, thus produced in the cascade, would be soft in the rest frame of $\tilde{\chi}_{2}^{0}$, it may be possible to tag hard photons in the lab frame. Note that the choice of light higgsinos are motivated by "naturalness" at the electroweak scale and we do not discuss the discovery potential for stop squarks and gluino in the present work which we plan to do in a subsequent extension.

\section{Conclusion}

To summarize, motivated by "naturalness" criteria at the electroweak scale, we have studied a simplified scenario with low $\mu$ parameter in the presence of a right-sneutrino LSP. For simplicity, we have assumed the gaugino mass parameters to be quite heavy $\gtrsim 1 \mathrm{TeV}$. In such a scenario, with $\mathcal{O}(100) \mathrm{GeV}$ Majorana mass parameter the neutrino Yukawa coupling can be as large as $10^{-6}-10^{-7}$. In contrast with the MSSM with light-higgsinos, in the present context, the higgsino-like states can decay to the sneutrino LSP. While the neutral higgsinos can decay into neutrino and sneutrino, the lightest chargino can decay into a 
lepton and sneutrino. We have demonstrated that the latter decay channel can lead to various leptonic final states with up to two leptons (i.e. mono-lepton, same-sign di-lepton and opposite-sign di-lepton) and missing transverse energy at the LHC, which can be important in searching for or constraining this scenario. We have only considered prompt decay into leptons, which require $y_{\nu}>10^{-7}$ and/or small $\mathcal{O}\left(10^{-5}-10^{-1}\right)$ left-right mixing in the sneutrino sector. For smaller values of $y_{\nu}$, contribution from the latter dominates and the leptonic partial width on small gaugino-higgsino mixing $\left(\lesssim \mathcal{O}\left(10^{-2}\right)\right)$. Further, the mass split between the three states, the lightest chargino and the two lightest neutralinos depend on the choice of the gaugino mass parameters, as well as on one-loop contributions. We have shown how these mass differences significantly affect the three-body partial widths, thus affecting the branching ratios to the sneutrino. Therefore, even assuming the gauginolike states to be above a $\mathrm{TeV}$, as in our benchmark scenarios, the viability of a low $\mu$ parameter depends crucially on the choice of $M_{1}, M_{2}$. This has been emphasized in great detail. Consequently, there are regions of the parameter space where $B R\left(\widetilde{\chi}_{1}^{ \pm} \rightarrow l \widetilde{\nu}\right) \sim 100 \%$ especially in the negative $M_{1}$ parameter space. Such regions of parameter space would lead to enhanced leptonic rates, thereby a large fraction of negative $M_{1}$ parameter space can be excluded from current leptonic searches at LHC. For a given $|\mu|$, we check the existing constraints by recasting our signal in CheckMATE against existing LHC analysis relevant for our model parameters to search for a viable parameter region of the model. We then choose some representative benchmarks and observe that mono-lepton signals with large $\mathbb{E}_{T}$ and little hadronic activity could successfully probe $\mu$ as low as $300 \mathrm{GeV}$ at the ongoing run of LHC with $106 \mathrm{fb}^{-1}$ of data at $3 \sigma$. Additional confirmatory channels for the $\widetilde{\nu}$ LSP scenario are opposite-sign di-lepton and same-sign di-lepton signal which require $\sim 50 \mathrm{fb}^{-1}$ and $\sim 800 \mathrm{fb}^{-1}$ for observing $3 \sigma$ excess at LHC. While our benchmarks assume the first two generations of sneutrinos to be degenerate and consider only $e, \mu$ for the charged leptons which can be detected efficiently at the LHC, the reach may be substantially reduced if only tau-sneutrino appears as the lightest flavor due to the low tau reconstruction efficiency.

\section{Acknowledgments}

AC acknowledges financial support from the Department of Science and Technology, Government of India through the INSPIRE Faculty Award: /2016/DST/INSPIRE/04 $/ 2015 / 000110$. The work of JD and SKR is partially supported by funding available from the Department of Atomic Energy, Government of India, for the Regional Centre for Accelerator-based Particle Physics (RECAPP), Harish-Chandra Research Institute. The authors would like to thank W. Porod for his help in providing several clarifications on our model implementation in SPheno and S. Choubey, S. Mondal and J. Beuria for useful discussions. Computational work for this work was carried out at the cluster computing facility (http://www.hri.res.in/cluster) and the RECAPP cluster in the Harish-Chandra Research Institute.

Open Access. This article is distributed under the terms of the Creative Commons Attribution License (CC-BY 4.0), which permits any use, distribution and reproduction in any medium, provided the original author(s) and source are credited. 


\section{References}

[1] R. Barbieri and G.F. Giudice, Upper Bounds on Supersymmetric Particle Masses, Nucl. Phys. B 306 (1988) 63 [inSPIRE].

[2] J.R. Ellis, K. Enqvist, D.V. Nanopoulos and F. Zwirner, Observables in Low-Energy Superstring Models, Mod. Phys. Lett. A 1 (1986) 57 [InSPIRE].

[3] J.L. Feng, Naturalness and the Status of Supersymmetry, Ann. Rev. Nucl. Part. Sci. 63 (2013) 351 [arXiv: 1302.6587] [INSPIRE].

[4] G.F. Giudice, Naturalness after LHC8, PoS(EPS-HEP 2013)163 [arXiv:1307.7879] [INSPIRE].

[5] H. Baer, V. Barger, P. Huang, D. Mickelson, A. Mustafayev and X. Tata, Radiative natural supersymmetry: Reconciling electroweak fine-tuning and the Higgs boson mass, Phys. Rev. D 87 (2013) 115028 [arXiv: 1212.2655] [INSPIRE].

[6] A. Mustafayev and X. Tata, Supersymmetry, Naturalness and Light Higgsinos, Indian J. Phys. 88 (2014) 991 [arXiv:1404.1386] [INSPIRE].

[7] H. Baer, V. Barger, P. Huang, A. Mustafayev and X. Tata, Radiative natural SUSY with a 125 GeV Higgs boson, Phys. Rev. Lett. 109 (2012) 161802 [arXiv:1207.3343] [InSPIRE].

[8] H. Baer, V. Barger, P. Huang, D. Mickelson, A. Mustafayev and X. Tata, Naturalness, Supersymmetry and Light Higgsinos: A Snowmass Whitepaper, in proceedings of 2013 Community Summer Study on the Future of U.S. Particle Physics: Snowmass on the Mississippi (CSS2013), Minneapolis, MN, U.S.A., July 29-August 6, 2013 [arXiv: 1306 . 2926] [INSPIRE].

[9] H. Baer, V. Barger and M. Savoy, Upper bounds on sparticle masses from naturalness or how to disprove weak scale supersymmetry, Phys. Rev. D 93 (2016) 035016 [arXiv:1509.02929] [INSPIRE].

[10] H. Baer et al., Gluino reach and mass extraction at the LHC in radiatively-driven natural SUSY, Eur. Phys. J. C 77 (2017) 499 [arXiv:1612.00795] [InSPIRE].

[11] ATLAS collaboration, https://atlas.web.cern.ch/Atlas/GROUPS/PHYSICS/CombinedSummaryPlots/SUSY/.

[12] CMS collaboration, https://twiki.cern.ch/twiki/bin/view/CMSPublic/PhysicsResultsSUS.

[13] A. Choudhury and A. Datta, Many faces of low mass neutralino dark matter in the unconstrained MSSM, LHC data and new signals, JHEP 06 (2012) 006 [arXiv:1203.4106] [INSPIRE].

[14] G. Bélanger, G. Drieu La Rochelle, B. Dumont, R.M. Godbole, S. Kraml and S. Kulkarni, LHC constraints on light neutralino dark matter in the MSSM, Phys. Lett. B 726 (2013) 773 [arXiv: 1308.3735] [INSPIRE].

[15] A. Choudhury and A. Datta, Neutralino dark matter confronted by the LHC constraints on Electroweak SUSY signals, JHEP 09 (2013) 119 [arXiv: 1305.0928] [INSPIRE].

[16] C. Boehm, P.S.B. Dev, A. Mazumdar and E. Pukartas, Naturalness of Light Neutralino Dark Matter in pMSSM after LHC, XENON100 and Planck Data, JHEP 06 (2013) 113 [arXiv: 1303.5386] [INSPIRE]. 
[17] M. Chakraborti, U. Chattopadhyay, A. Choudhury, A. Datta and S. Poddar, The Electroweak Sector of the pMSSM in the Light of LHC $-8 \mathrm{TeV}$ and Other Data, JHEP 07 (2014) 019 [arXiv: 1404.4841] [INSPIRE].

[18] G. Grilli di Cortona, Hunting electroweakinos at future hadron colliders and direct detection experiments, JHEP 05 (2015) 035 [arXiv: 1412.5952] [INSPIRE].

[19] M. Drees and J.S. Kim, Minimal natural supersymmetry after the LHC8, Phys. Rev. D 93 (2016) 095005 [arXiv: 1511.04461] [INSPIRE].

[20] M. Chakraborti, U. Chattopadhyay, A. Choudhury, A. Datta and S. Poddar, Reduced LHC constraints for higgsino-like heavier electroweakinos, JHEP 11 (2015) 050

[arXiv:1507.01395] [inSPIRE].

[21] M. Badziak, A. Delgado, M. Olechowski, S. Pokorski and K. Sakurai, Detecting underabundant neutralinos, JHEP 11 (2015) 053 [arXiv:1506.07177] [INSPIRE].

[22] J. Cao, Y. He, L. Shang, W. Su and Y. Zhang, Testing the light dark matter scenario of the MSSM at the LHC, JHEP 03 (2016) 207 [arXiv: 1511.05386] [INSPIRE].

[23] M. Beneke, A. Bharucha, A. Hryczuk, S. Recksiegel and P. Ruiz-Femenia, The last refuge of mixed wino-Higgsino dark matter, JHEP 01 (2017) 002 [arXiv:1611.00804] [INSPIRE].

[24] H. Baer, V. Barger, P. Huang, D. Mickelson, M. Padeffke-Kirkland and X. Tata, Natural SUSY with a bino- or wino-like LSP, Phys. Rev. D 91 (2015) 075005 [arXiv:1501.06357] [INSPIRE].

[25] M. Abdughani, L. Wu and J.M. Yang, Status and prospects of light bino-higgsino dark matter in natural SUSY, Eur. Phys. J. C 78 (2018) 4 [arXiv:1705.09164] [INSPIRE].

[26] M.R. Buckley, D. Feld, S. Macaluso, A. Monteux and D. Shih, Cornering Natural SUSY at LHC Run II and Beyond, JHEP 08 (2017) 115 [arXiv: 1610.08059] [INSPIRE].

[27] H. Baer, V. Barger and P. Huang, Hidden SUSY at the LHC: the light higgsino-world scenario and the role of a lepton collider, JHEP 11 (2011) 031 [arXiv:1107.5581] [INSPIRE].

[28] C. Han, A. Kobakhidze, N. Liu, A. Saavedra, L. Wu and J.M. Yang, Probing Light Higgsinos in Natural SUSY from Monojet Signals at the LHC, JHEP 02 (2014) 049 [arXiv: 1310.4274$]$ [INSPIRE].

[29] Z. Han, G.D. Kribs, A. Martin and A. Menon, Hunting quasidegenerate Higgsinos, Phys. Rev. D 89 (2014) 075007 [arXiv: 1401.1235] [inSPIRE].

[30] D. Barducci, A. Belyaev, A.K.M. Bharucha, W. Porod and V. Sanz, Uncovering Natural Supersymmetry via the interplay between the LHC and Direct Dark Matter Detection, JHEP 07 (2015) 066 [arXiv: 1504.02472] [INSPIRE].

[31] H. Baer, V. Barger, M. Savoy and X. Tata, Multichannel assault on natural supersymmetry at the high luminosity LHC, Phys. Rev. D 94 (2016) 035025 [arXiv: 1604. 07438] [InSPIRE].

[32] H. Fukuda, N. Nagata, H. Otono and S. Shirai, Higgsino Dark Matter or Not: Role of Disappearing Track Searches at the LHC and Future Colliders, Phys. Lett. B 781 (2018) 306 [arXiv: 1703.09675] [INSPIRE].

[33] R. Mahbubani, P. Schwaller and J. Zurita, Closing the window for compressed Dark Sectors with disappearing charged tracks, JHEP 06 (2017) 119 [Erratum ibid. 10 (2017) 061] [arXiv: 1703.05327] [INSPIRE]. 
[34] S. Ambrosanio, B. Mele, S. Petrarca, G. Polesello and A. Rimoldi, Measuring the SUSY breaking scale at the LHC in the slepton NLSP scenario of GMSB models, JHEP 01 (2001) 014 [hep-ph/0010081] [INSPIRE].

[35] J.R. Ellis, K.A. Olive, Y. Santoso and V.C. Spanos, Prospects for sparticle discovery in variants of the MSSM, Phys. Lett. B 603 (2004) 51 [hep-ph/0408118] [InSPIRE].

[36] L. Roszkowski, R. Ruiz de Austri and K.-Y. Choi, Gravitino dark matter in the CMSSM and implications for leptogenesis and the LHC, JHEP 08 (2005) 080 [hep-ph/0408227] [INSPIRE].

[37] J.R. Ellis, A.R. Raklev and O.K. Oye, Gravitino dark matter scenarios with massive metastable charged sparticles at the LHC, JHEP 10 (2006) 061 [hep-ph/0607261] [INSPIRE].

[38] G.D. Kribs, A. Martin and T.S. Roy, Supersymmetry with a Chargino NLSP and Gravitino LSP, JHEP 01 (2009) 023 [arXiv:0807.4936] [INSPIRE].

[39] S. Bailly, K.-Y. Choi, K. Jedamzik and L. Roszkowski, A Re-analysis of Gravitino Dark Matter in the Constrained MSSM, JHEP 05 (2009) 103 [arXiv:0903.3974] [INSPIRE].

[40] J.L. Feng, M. Kamionkowski and S.K. Lee, Light Gravitinos at Colliders and Implications for Cosmology, Phys. Rev. D 82 (2010) 015012 [arXiv: 1004.4213] [INSPIRE].

[41] T. Figy, K. Rolbiecki and Y. Santoso, Tau-Sneutrino NLSP and Multilepton Signatures at the LHC, Phys. Rev. D 82 (2010) 075016 [arXiv: 1005.5136] [InSPIRE].

[42] P. de Aquino, F. Maltoni, K. Mawatari and B. Oexl, Light Gravitino Production in Association with Gluinos at the LHC, JHEP 10 (2012) 008 [arXiv:1206.7098] [INSPIRE].

[43] J. Barnard, B. Farmer, T. Gherghetta and M. White, Natural gauge mediation with a bino NLSP at the LHC, Phys. Rev. Lett. 109 (2012) 241801 [arXiv:1208.6062] [InSPIRE].

[44] S. Bobrovskyi, J. Hajer and S. Rydbeck, Long-lived higgsinos as probes of gravitino dark matter at the LHC, JHEP 02 (2013) 133 [arXiv:1211.5584] [INSPIRE].

[45] L. Roszkowski, S. Trojanowski, K. Turzynski and K. Jedamzik, Gravitino dark matter with constraints from Higgs boson mass and sneutrino decays, JHEP 03 (2013) 013 [arXiv:1212.5587] [INSPIRE].

[46] R.H. Cyburt, J. Ellis, B.D. Fields, F. Luo, K.A. Olive and V.C. Spanos, Gravitino Decays and the Cosmological Lithium Problem in Light of the LHC Higgs and Supersymmetry Searches, JCAP 05 (2013) 014 [arXiv: 1303.0574] [INSPIRE].

[47] J. D'Hondt et al., Multilepton signals of gauge mediated supersymmetry breaking at the LHC, Phys. Lett. B 731 (2014) 7 [arXiv:1310.0018] [INSPIRE].

[48] J. Heisig, Gravitino LSP and leptogenesis after the first LHC results, JCAP 04 (2014) 023 [arXiv:1310.6352] [INSPIRE].

[49] L. Covi and F. Dradi, Long-Lived stop at the LHC with or without R-parity, JCAP 10 (2014) 039 [arXiv: 1403.4923] [inSPIRE].

[50] F. Maltoni, A. Martini, K. Mawatari and B. Oexl, Signals of a superlight gravitino at the LHC, JHEP 04 (2015) 021 [arXiv: 1502.01637] [INSPIRE].

[51] A. Arbey, M. Battaglia, L. Covi, J. Hasenkamp and F. Mahmoudi, LHC constraints on Gravitino Dark Matter, Phys. Rev. D 92 (2015) 115008 [arXiv:1505.04595] [InSPIRE].

[52] J.S. Kim, M.E. Krauss and V. Martin-Lozano, Probing the Electroweakino Sector of General Gauge Mediation at the LHC, arXiv:1705.06497 [INSPIRE]. 
[53] J. Dutta, P. Konar, S. Mondal, B. Mukhopadhyaya and S.K. Rai, Search for a compressed supersymmetric spectrum with a light Gravitino, JHEP 09 (2017) 026 [arXiv:1704.04617] [INSPIRE].

[54] H. Baer, R. Dermisek, S. Rajagopalan and H. Summy, Neutralino, axion and axino cold dark matter in minimal, hypercharged and gaugino AMSB, JCAP 07 (2010) 014 [arXiv: 1004.3297] [INSPIRE].

[55] H. Baer, A. Lessa, S. Rajagopalan and W. Sreethawong, Mixed axion/neutralino cold dark matter in supersymmetric models, JCAP 06 (2011) 031 [arXiv:1103.5413] [INSPIRE].

[56] K.J. Bae, H. Baer and E.J. Chun, Mixed axion/neutralino dark matter in the SUSY DFSZ axion model, JCAP 12 (2013) 028 [arXiv:1309.5365] [INSPIRE].

[57] K.J. Bae, H. Baer, E.J. Chun and C.S. Shin, Mixed axion/gravitino dark matter from SUSY models with heavy axinos, Phys. Rev. D 91 (2015) 075011 [arXiv:1410.3857] [InSPIRE].

[58] K.J. Bae, H. Baer, A. Lessa and H. Serce, Mixed axion-wino dark matter, Front. in Phys. 3 (2015) 49 [arXiv: 1502.07198] [INSPIRE].

[59] N. Arkani-Hamed, L.J. Hall, H. Murayama, D. Tucker-Smith and N. Weiner, Small neutrino masses from supersymmetry breaking, Phys. Rev. D 64 (2001) 115011 [hep-ph/0006312] [INSPIRE].

[60] S. Gopalakrishna, A. de Gouvêa and W. Porod, Right-handed sneutrinos as nonthermal dark matter, JCAP 05 (2006) 005 [hep-ph/0602027] [INSPIRE].

[61] T. Asaka, K. Ishiwata and T. Moroi, Right-handed sneutrino as cold dark matter, Phys. Rev. D 73 (2006) 051301 [hep-ph/0512118] [INSPIRE].

[62] T. Asaka, K. Ishiwata and T. Moroi, Right-handed sneutrino as cold dark matter of the universe, Phys. Rev. D 75 (2007) 065001 [hep-ph/0612211] [InSPIRE].

[63] V. Page, Non-thermal right-handed sneutrino dark matter and the Omega(DM)/Omega(b) problem, JHEP 04 (2007) 021 [hep-ph/0701266] [INSPIRE].

[64] A. Kumar, D. Tucker-Smith and N. Weiner, Neutrino Mass, Sneutrino Dark Matter and Signals of Lepton Flavor Violation in the MRSSM, JHEP 09 (2010) 111 [arXiv:0910.2475] [INSPIRE].

[65] K. Kadota and K.A. Olive, Heavy Right-Handed Neutrinos and Dark Matter in the nuCMSSM, Phys. Rev. D 80 (2009) 095015 [arXiv: 0909.3075] [inSPIRE].

[66] G. Bélanger, M. Kakizaki, E.K. Park, S. Kraml and A. Pukhov, Light mixed sneutrinos as thermal dark matter, JCAP 11 (2010) 017 [arXiv: 1008.0580] [INSPIRE].

[67] B. Dumont, G. Bélanger, S. Fichet, S. Kraml and T. Schwetz, Mixed sneutrino dark matter in light of the 2011 XENON and LHC results, JCAP 09 (2012) 013 [arXiv:1206.1521] [INSPIRE].

[68] M. Kakizaki, E.-K. Park, J.-h. Park and A. Santa, Phenomenological constraints on light mixed sneutrino dark matter scenarios, Phys. Lett. B 749 (2015) 44 [arXiv: 1503.06783] [INSPIRE].

[69] A. Chatterjee, D. Das, B. Mukhopadhyaya and S.K. Rai, Right Sneutrino Dark Matter and a Monochromatic Photon Line, JCAP 07 (2014) 023 [arXiv: 1401.2527] [INSPIRE].

[70] N. Cerna-Velazco, T. Faber, J. Jones-Perez and W. Porod, Constraining sleptons at the LHC in a supersymmetric low-scale seesaw scenario, Eur. Phys. J. C 77 (2017) 661 [arXiv: 1705. 06583] [INSPIRE]. 
[71] T. Falk, K.A. Olive and M. Srednicki, Heavy sneutrinos as dark matter, Phys. Lett. B 339 (1994) 248 [hep-ph/9409270] [INSPIRE].

[72] C. Arina and N. Fornengo, Sneutrino cold dark matter, a new analysis: Relic abundance and detection rates, JHEP 11 (2007) 029 [arXiv:0709.4477] [INSPIRE].

[73] A. de Gouvêa, S. Gopalakrishna and W. Porod, Stop Decay into Right-handed Sneutrino LSP at Hadron Colliders, JHEP 11 (2006) 050 [hep-ph/0606296] [INSPIRE].

[74] S.K. Gupta, B. Mukhopadhyaya and S.K. Rai, Right-chiral sneutrinos and long-lived staus: Event characteristics at the large hadron collider, Phys. Rev. D 75 (2007) 075007 [hep-ph/0701063] [inSPIRE].

[75] D. Choudhury, S.K. Gupta and B. Mukhopadhyaya, Right sneutrinos in a supergravity model and the signals of a stable stop at the Large Hadron Collider, Phys. Rev. D 78 (2008) 015023 [arXiv: 0804.3560] [INSPIRE].

[76] J. Guo, Z. Kang, J. Li, T. Li and Y. Liu, Simplified Supersymmetry with Sneutrino LSP at $8 \mathrm{TeV}$ LHC, JHEP 10 (2014) 164 [arXiv:1312.2821] [INSPIRE].

[77] C. Arina and M.E. Cabrera, Multi-lepton signatures at LHC from sneutrino dark matter, JHEP 04 (2014) 100 [arXiv:1311.6549] [INSPIRE].

[78] C. Arina, M.E.C. Catalan, S. Kraml, S. Kulkarni and U. Laa, Constraints on sneutrino dark matter from LHC Run 1, JHEP 05 (2015) 142 [arXiv:1503.02960] [INSPIRE].

[79] S. Banerjee, G. Bélanger, B. Mukhopadhyaya and P.D. Serpico, Signatures of sneutrino dark matter in an extension of the CMSSM, JHEP 07 (2016) 095 [arXiv:1603.08834] [INSPIRE].

[80] Y. Grossman and H.E. Haber, Sneutrino mixing phenomena, Phys. Rev. Lett. 78 (1997) 3438 [hep-ph/9702421] [INSPIRE].

[81] P. Minkowski, $\mu \rightarrow$ ey at a Rate of One Out of $10^{9}$ Muon Decays?, Phys. Lett. B 67 (1977) 421 [INSPIRE].

[82] T. Yanagida, Horizontal symmetry and masses of neutrinos, Conf. Proc. C 7902131 (1979) 95 [INSPIRE].

[83] R.N. Mohapatra and G. Senjanović, Neutrino Mass and Spontaneous Parity Violation, Phys. Rev. Lett. 44 (1980) 912 [INSPIRE].

[84] J.A. Casas and A. Ibarra, Oscillating neutrinos and $\mu \rightarrow e, \gamma$, Nucl. Phys. B 618 (2001) 171 [hep-ph/0103065] [INSPIRE].

[85] M. Drewes, The Phenomenology of Right Handed Neutrinos, Int. J. Mod. Phys. E 22 (2013) 1330019 [arXiv:1303.6912] [INSPIRE].

[86] R.W. Rasmussen and W. Winter, Perspectives for tests of neutrino mass generation at the GeV scale: Experimental reach versus theoretical predictions, Phys. Rev. D 94 (2016) 073004 [arXiv: 1607.07880] [INSPIRE].

[87] A. Dedes, H.E. Haber and J. Rosiek, Seesaw mechanism in the sneutrino sector and its consequences, JHEP 11 (2007) 059 [arXiv:0707.3718] [INSPIRE].

[88] M. Drees, R. Godbole and P. Roy, Theory and phenomenology of sparticles: An account of four-dimensional $N=1$ supersymmetry in high energy physics, World Scientific (2004) [INSPIRE]. 
[89] M. Drees, M.M. Nojiri, D.P. Roy and Y. Yamada, Light Higgsino dark matter, Phys. Rev. D 56 (1997) 276 [Erratum ibid. D 64 (2001) 039901] [hep-ph/9701219] [INSPIRE].

[90] G.F. Giudice and A. Pomarol, Mass degeneracy of the Higgsinos, Phys. Lett. B 372 (1996) 253 [hep-ph/9512337] [INSPIRE].

[91] D. Pierce and A. Papadopoulos, Radiative corrections to neutralino and chargino masses in the minimal supersymmetric model, Phys. Rev. D 50 (1994) 565 [hep-ph/9312248] [INSPIRE].

[92] D. Pierce and A. Papadopoulos, The Complete radiative corrections to the gaugino and Higgsino masses in the minimal supersymmetric model, Nucl. Phys. B 430 (1994) 278 [hep-ph/9403240] [INSPIRE].

[93] A.B. Lahanas, K. Tamvakis and N.D. Tracas, One loop corrections to the neutralino sector and radiative electroweak breaking in the MSSM, Phys. Lett. B 324 (1994) 387 [hep-ph/9312251] [INSPIRE].

[94] F. Staub, SARAH, arXiv:0806.0538 [inSPIRE].

[95] F. Staub, SARAH 4: A tool for (not only SUSY) model builders, Comput. Phys. Commun. 185 (2014) 1773 [arXiv:1309.7223] [inSPIRE].

[96] W. Porod, SPheno, a program for calculating supersymmetric spectra, SUSY particle decays and SUSY particle production at $e^{+} e^{-}$colliders, Comput. Phys. Commun. 153 (2003) 275 [hep-ph/0301101] [INSPIRE].

[97] W. Porod and F. Staub, SPheno 3.1: Extensions including flavour, CP-phases and models beyond the MSSM, Comput. Phys. Commun. 183 (2012) 2458 [arXiv:1104.1573] [INSPIRE].

[98] P.Z. Skands et al., SUSY Les Houches accord: Interfacing SUSY spectrum calculators, decay packages and event generators, JHEP 07 (2004) 036 [hep-ph/0311123] [INSPIRE].

[99] A. Djouadi, Y. Mambrini and M. Muhlleitner, Chargino and neutralino decays revisited, Eur. Phys. J. C 20 (2001) 563 [hep-ph/0104115] [INSPIRE].

[100] C.H. Chen, M. Drees and J.F. Gunion, Searching for invisible and almost invisible particles at $e^{+} e^{-}$colliders, Phys. Rev. Lett. 76 (1996) 2002 [hep-ph/9512230] [INSPIRE].

[101] C.H. Chen, M. Drees and J.F. Gunion, A Nonstandard string/SUSY scenario and its phenomenological implications, Phys. Rev. D 55 (1997) 330 [Erratum ibid. D 60 (1999) 039901] [hep-ph/9607421] [INSPIRE].

[102] C.H. Chen, M. Drees and J.F. Gunion, Addendum/erratum for 'searching for invisible and almost invisible particles at $e^{+} e^{-}$colliders' [hep-ph/9512230] and 'a nonstandard string/SUSY scenario and its phenomenological implications' [hep-ph/9607421], hep-ph/9902309 [INSPIRE].

[103] H.E. Haber and D. Wyler, Radiative neutralino decay, Nucl. Phys. B 323 (1989) 267 [INSPIRE].

[104] S. Ambrosanio and B. Mele, Neutralino decays in the minimal supersymmetric Standard Model, Phys. Rev. D 53 (1996) 2541 [hep-ph/9508237] [INSPIRE].

[105] S. Ambrosanio and B. Mele, Supersymmetric scenarios with dominant radiative neutralino decay, Phys. Rev. D 55 (1997) 1399 [Erratum ibid. D 56 (1997) 3157] [hep-ph/9609212] [INSPIRE]. 
[106] H. Baer and T. Krupovnickas, Radiative neutralino decay in supersymmetric models, JHEP 09 (2002) 038 [hep-ph/0208277] [INSPIRE].

[107] ATLAS, CMS collaborations, Combined Measurement of the Higgs Boson Mass in pp Collisions at $\sqrt{s}=7$ and 8 TeV with the ATLAS and CMS Experiments, Phys. Rev. Lett. 114 (2015) 191803 [arXiv: 1503.07589] [INSPIRE].

[108] ATLAS collaboration, Observation of a new particle in the search for the Standard Model Higgs boson with the ATLAS detector at the LHC, Phys. Lett. B 716 (2012) 1 [arXiv:1207.7214] [INSPIRE].

[109] CMS collaboration, Observation of a new boson at a mass of $125 \mathrm{GeV}$ with the CMS experiment at the LHC, Phys. Lett. B 716 (2012) 30 [arXiv:1207.7235] [INSPIRE].

[110] M. Carena, S. Heinemeyer, O. Stål, C.E.M. Wagner and G. Weiglein, MSSM Higgs Boson Searches at the LHC: Benchmark Scenarios after the Discovery of a Higgs-like Particle, Eur. Phys. J. C 73 (2013) 2552 [arXiv:1302.7033] [INSPIRE].

[111] LEP2SUSYWG et al., Lep2 joint susy working group, [http://lepsusy.web.cern.ch/lepsusy/] [http://lepsusy.web.cern.ch/lepsusy/www/inoslowdmsummer02/charginolowdm_pub.html] [http://lepsusy.web.cern.ch/lepsusy/www/inos_moriond01/charginos_pub.html].

[112] CMS collaboration, Searches for invisible decays of the Higgs boson in pp collisions at $\sqrt{s}=7,8$ and $13 \mathrm{TeV}$, JHEP 02 (2017) 135 [arXiv:1610.09218] [INSPIRE].

[113] Particle Data Group collaboration, C. Patrignani et al., Review of Particle Physics, Chin. Phys. C 40 (2016) 100001 [inSPIRE].

[114] LHCb collaboration, First Evidence for the Decay $B_{s}^{0} \rightarrow \mu^{+} \mu^{-}$, Phys. Rev. Lett. 110 (2013) 021801 [arXiv: 1211.2674] [INSPIRE].

[115] Heavy Flavor Averaging Group collaboration, Y. Amhis et al., Averages of B-Hadron, C-Hadron and tau-lepton properties as of early 2012, arXiv:1207.1158 [INSPIRE].

[116] Planck collaboration, P.A.R. Ade et al., Planck 2015 results. XIII. Cosmological parameters, Astron. Astrophys. 594 (2016) A13 [arXiv:1502.01589] [INSPIRE].

[117] LUX collaboration, D.S. Akerib et al., Results from a search for dark matter in the complete LUX exposure, Phys. Rev. Lett. 118 (2017) 021303 [arXiv: 1608. 07648] [INSPIRE].

[118] PandaX-II collaboration, A. Tan et al., Dark Matter Results from First 98.7 Days of Data from the PandaX-II Experiment, Phys. Rev. Lett. 117 (2016) 121303 [arXiv:1607.07400] [INSPIRE].

[119] XENON collaboration, E. Aprile et al., First Dark Matter Search Results from the XENON1T Experiment, Phys. Rev. Lett. 119 (2017) 181301 [arXiv:1705.06655] [INSPIRE].

[120] G. Bélanger, F. Boudjema, A. Pukhov and A. Semenov, MicrOMEGAs_3: A program for calculating dark matter observables, Comput. Phys. Commun. 185 (2014) 960 [arXiv: 1305.0237] [INSPIRE].

[121] L.J. Hall, T. Moroi and H. Murayama, Sneutrino cold dark matter with lepton number violation, Phys. Lett. B 424 (1998) 305 [hep-ph/9712515] [INSPIRE].

[122] D. Tucker-Smith and N. Weiner, Inelastic dark matter, Phys. Rev. D 64 (2001) 043502 [hep-ph/0101138] [INSPIRE].

[123] H. Baer, K.-Y. Choi, J.E. Kim and L. Roszkowski, Dark matter production in the early Universe: beyond the thermal WIMP paradigm, Phys. Rept. 555 (2015) 1

[arXiv:1407.0017] [INSPIRE]. 
[124] B.S. Acharya, P. Kumar, K. Bobkov, G. Kane, J. Shao and S. Watson, Non-thermal Dark Matter and the Moduli Problem in String Frameworks, JHEP 06 (2008) 064 [arXiv:0804.0863] [INSPIRE].

[125] ATLAS collaboration, Search for long-lived, massive particles in events with displaced vertices and missing transverse momentum in $\sqrt{s}=13$ TeV pp collisions with the ATLAS detector, Phys. Rev. D 97 (2018) 052012 [arXiv:1710.04901] [INSPIRE].

[126] W. Beenakker, R. Hopker and M. Spira, PROSPINO: A Program for the production of supersymmetric particles in next-to-leading order QCD, hep-ph/9611232 [INSPIRE].

[127] T. Plehn, Measuring the MSSM Lagrangean, Czech. J. Phys. 55 (2005) B213 [hep-ph/0410063] [INSPIRE].

[128] M. Spira, Higgs and SUSY particle production at hadron colliders, in Supersymmetry and unification of fundamental interactions. Proceedings, 10th International Conference, SUSY'02, Hamburg, Germany, June 17-23, 2002, pp. 217-226 [hep-ph/0211145] [INSPIRE].

[129] CMS collaboration, Search for electroweak production of charginos and neutralinos in multilepton final states in pp collision data at $\sqrt{s}=13$ TeV, CMS-PAS-SUS-16-039 (2017).

[130] CMS collaboration, Search for new physics in events with two low momentum opposite-sign leptons and missing transverse energy at $\sqrt{s}=13 \mathrm{TeV}$, CMS-PAS-SUS-16-048 (2017).

[131] ATLAS collaboration, Search for electroweak production of supersymmetric states in scenarios with compressed mass spectra at $\sqrt{s}=13 \mathrm{TeV}$ with the ATLAS detector, Phys. Rev. D 97 (2018) 052010 [arXiv: 1712.08119] [INSPIRE].

[132] ATLAS collaboration, Search for electroweak production of supersymmetric particles in the two and three lepton final state at $\sqrt{s}=13 \mathrm{TeV}$ with the ATLAS detector, ATLAS-CONF-2017-039 (2017).

[133] ATLAS collaboration, Search for direct production of charginos and neutralinos in events with three leptons and missing transverse momentum in $\sqrt{s}=8 T e V$ pp collisions with the ATLAS detector, JHEP 04 (2014) 169 [arXiv: 1402.7029] [INSPIRE].

[134] ATLAS collaboration, Search for direct top-squark pair production in final states with two leptons in pp collisions at $\sqrt{s}=8 \mathrm{TeV}$ with the ATLAS detector, JHEP 06 (2014) 124 [arXiv: 1403.4853] [INSPIRE].

[135] ATLAS collaboration, Search for direct top squark pair production in events with a $Z$ boson, b-jets and missing transverse momentum in $\sqrt{s}=8 \mathrm{TeV}$ pp collisions with the ATLAS detector, Eur. Phys. J. C 74 (2014) 2883 [arXiv:1403.5222] [INSPIRE].

[136] ATLAS collaboration, Search for supersymmetry at $\sqrt{s}=8 \mathrm{TeV}$ in final states with jets and two same-sign leptons or three leptons with the ATLAS detector, JHEP 06 (2014) 035 [arXiv: 1404.2500] [INSPIRE].

[137] ATLAS collaboration, Search for top squark pair production in final states with one isolated lepton, jets and missing transverse momentum in $\sqrt{s}=8 \mathrm{TeV}$ pp collisions with the ATLAS detector, JHEP 11 (2014) 118 [arXiv:1407.0583] [INSPIRE].

[138] ATLAS collaboration, Search for supersymmetry in events containing a same-flavour opposite-sign dilepton pair, jets and large missing transverse momentum in $\sqrt{s}=8 \mathrm{TeV} \mathrm{pp}$ collisions with the ATLAS detector, Eur. Phys. J. C 75 (2015) 318 [arXiv:1503.03290] [INSPIRE]. 
[139] ATLAS collaboration, Search for new phenomena in final states with an energetic jet and large missing transverse momentum in pp collisions at $\sqrt{s}=8 \mathrm{TeV}$ with the ATLAS detector, Eur. Phys. J. C 75 (2015) 299 [arXiv: 1502.01518] [INSPIRE].

[140] CMS collaboration, Search for dark matter, extra dimensions and unparticles in monojet events in proton-proton collisions at $\sqrt{s}=8$ TeV, Eur. Phys. J. C 75 (2015) 235 [arXiv: 1408.3583] [INSPIRE].

[141] ATLAS collaboration, ATLAS Run 1 searches for direct pair production of third-generation squarks at the Large Hadron Collider, Eur. Phys. J. C 75 (2015) 510 [arXiv:1506.08616] [INSPIRE].

[142] ATLAS collaboration, Search for supersymmetry at $\sqrt{s}=8 \mathrm{TeV}$ in final states with jets, missing transverse momentum and one isolated lepton, ATLAS-CONF-2012-104 (2012).

[143] ATLAS collaboration, Search for direct production of charginos and neutralinos in events with three leptons and missing transverse momentum in $21 \mathrm{fb}^{-1}$ of $\mathrm{pp}$ collisions at $\sqrt{s}=8 \mathrm{TeV}$ with the ATLAS detector, ATLAS-CONF-2013-035 (2013).

[144] ATLAS collaboration, Search for direct-slepton and direct-chargino production in final states with two opposite-sign leptons, missing transverse momentum and no jets in $20 / f b$ of pp collisions at $\sqrt{s}=8 \mathrm{TeV}$ with the ATLAS detector, ATLAS-CONF-2013-049 (2013).

[145] ATLAS collaboration, Search for strong production of supersymmetric particles in final states with missing transverse momentum and at least three b-jets using $20.1 \mathrm{fb}^{-1}$ of $p p$ collisions at $\sqrt{s}=8 \mathrm{TeV}$ with the ATLAS Detector, ATLAS-CONF-2013-061 (2013).

[146] ATLAS collaboration, Search for strongly produced supersymmetric particles in decays with two leptons at $\sqrt{s}=8 \mathrm{TeV}$, ATLAS-CONF-2013-089 (2013).

[147] CMS collaboration, Search for Physics Beyond the Standard Model in Events with Two Leptons, Jets and Missing Transverse Momentum in pp Collisions at $\sqrt{s}=8$ TeV, JHEP 04 (2015) 124 [arXiv: 1502.06031] [INSPIRE].

[148] CMS collaboration, Search for the production of dark matter in association with top-quark pairs in the single-lepton final state in proton-proton collisions at $\sqrt{s}=8 \mathrm{TeV}, J H E P 06$ (2015) 121 [arXiv: 1504.03198] [INSPIRE].

[149] CMS collaboration, CMS Collaboration, CMS-PAS-SUS-13-016 (CMS Collaboration).

[150] ATLAS collaboration, Search for supersymmetry at $\sqrt{s}=13 \mathrm{TeV}$ in final states with jets and two same-sign leptons or three leptons with the ATLAS detector, Eur. Phys. J. C 76 (2016) 259 [arXiv: 1602.09058] [inSPIRE].

[151] ATLAS collaboration, Search for new phenomena in final states with an energetic jet and large missing transverse momentum in pp collisions at $\sqrt{s}=13$ TeV using the ATLAS detector, Phys. Rev. D 94 (2016) 032005 [arXiv: 1604.07773] [INSPIRE].

[152] ATLAS collaboration, Search for gluinos in events with an isolated lepton, jets and missing transverse momentum at $\sqrt{s}=13 \mathrm{TeV}$ with the ATLAS detector, Eur. Phys. J. C 76 (2016) 565 [arXiv: 1605.04285] [INSPIRE].

[153] ATLAS collaboration, Search for pair production of gluinos decaying via stop and sbottom in events with $b$-jets and large missing transverse momentum in pp collisions at $\sqrt{s}=13$ TeV with the ATLAS detector, Phys. Rev. D 94 (2016) 032003 [arXiv:1605.09318] [INSPIRE]. 
[154] ATLAS collaboration, Search for top squarks in final states with one isolated lepton, jets and missing transverse momentum in $\sqrt{s}=13 \mathrm{TeV}$ pp collisions with the ATLAS detector, Phys. Rev. D 94 (2016) 052009 [arXiv:1606.03903] [InSPIRE].

[155] ATLAS collaboration, A search for Supersymmetry in events containing a leptonically decaying $Z$ boson, jets and missing transverse momentum in $\sqrt{s}=13 \mathrm{TeV}$ pp collisions with the ATLAS detector, ATLAS-CONF-2015-082 (2015).

[156] ATLAS collaboration, Search for production of vector-like quark pairs and of four top quarks in the lepton-plus-jets final state in pp collisions at $\sqrt{s}=8 \mathrm{TeV}$ with the ATLAS detector, JHEP 08 (2015) 105 [arXiv: 1505.04306] [INSPIRE].

[157] ATLAS collaboration, Search for top-squark pair production in final states with one lepton, jets and missing transverse momentum using $36 \mathrm{fb}^{-1}$ of $\sqrt{s}=13 \mathrm{TeV}$ pp collision data with the ATLAS detector, arXiv:1711.11520 [INSPIRE].

[158] ATLAS collaboration, Search for top squarks in final states with one isolated lepton, jets and missing transverse momentum in $\sqrt{s}=13 \mathrm{TeV}$ pp collisions with the ATLAS detector, ATLAS-CONF-2016-050 (2016).

[159] CMS collaboration, Search for new physics in final states with two opposite-sign, same-flavor leptons, jets and missing transverse momentum in pp collisions at $\sqrt{s}=13 \mathrm{TeV}$, JHEP 12 (2016) 013 [arXiv: 1607.00915] [INSPIRE].

[160] ATLAS collaboration, Search for dark matter and other new phenomena in events with an energetic jet and large missing transverse momentum using the ATLAS detector, JHEP 01 (2018) 126 [arXiv: 1711.03301] [INSPIRE].

[161] ATLAS collaboration, Search for a new heavy gauge boson resonance decaying into a lepton and missing transverse momentum in $36 \mathrm{fb}^{-1}$ of pp collisions at $\sqrt{\mathrm{s}}=13 \mathrm{TeV}$ with the ATLAS experiment, arXiv:1706.04786 [INSPIRE].

[162] CMS collaboration, Search for heavy gauge $W^{\prime}$ boson in events with an energetic lepton and large missing transverse momentum at $\sqrt{s}=13$ TeV, Phys. Lett. B 770 (2017) 278 [arXiv: 1612.09274] [INSPIRE].

[163] CMS collaboration, Search for supersymmetry in events with at least one soft lepton, low jet multiplicity and missing transverse momentum in proton-proton collisions at $\sqrt{s}=13 \mathrm{TeV}$, CMS-PAS-SUS-16-052 (2017).

[164] CMS collaboration, Search for narrow resonances in dilepton mass spectra in proton-proton collisions at $\sqrt{s}=13 \mathrm{TeV}$ and combination with $8 \mathrm{TeV}$ data, Phys. Lett. B 768 (2017) 57 [arXiv: 1609.05391] [INSPIRE].

[165] CMS collaboration, Search for selectrons and smuons at $\sqrt{s}=13 \mathrm{TeV}$, CMS-PAS-SUS-17-009 (2017).

[166] CMS collaboration, Search for supersymmetry in events with tau leptons and missing transverse momentum in proton-proton collisions at $\sqrt{s}=13 \mathrm{TeV}$, CMS-PAS-SUS-17-002 (2017).

[167] CMS collaboration, Search for electroweak production of charginos and neutralinos in multilepton final states in proton-proton collisions at $\sqrt{s}=13 \mathrm{TeV}$, JHEP 03 (2018) 166 [arXiv: 1709.05406] [INSPIRE].

[168] ATLAS collaboration, Search for supersymmetry in final states with two same-sign or three leptons and jets using $36 \mathrm{fb}^{-1}$ of $\sqrt{\mathrm{s}}=13 \mathrm{TeV}$ pp collision data with the ATLAS detector, JHEP 09 (2017) 084 [arXiv:1706.03731] [INSPIRE]. 
[169] ATLAS collaboration, Search for supersymmetry in events with four or more leptons in $\sqrt{s}=13 \mathrm{TeV}$ pp collisions using $13.3 \mathrm{fb}^{-1}$ of ATLAS data., ATLAS-CONF-2016-075 (2016).

[170] CMS collaboration, Combined search for electroweak production of charginos and neutralinos in proton-proton collisions at $\sqrt{s}=13$ TeV, JHEP 03 (2018) 160 [arXiv: 1801.03957] [INSPIRE].

[171] CMS collaboration, Search for new phenomena with multiple charged leptons in proton-proton collisions at $\sqrt{s}=13$ TeV, Eur. Phys. J. C 77 (2017) 635 [arXiv:1701.06940] [INSPIRE].

[172] CMS collaboration, Search for supersymmetry in events with soft leptons, low jet multiplicity and missing transverse energy in proton-proton collisions at $\sqrt{s}=8 \mathrm{TeV}$, Phys. Lett. B 759 (2016) 9 [arXiv:1512.08002] [INSPIRE].

[173] H. Beauchesne, E. Bertuzzo, G. Grilli Di Cortona and Z. Tabrizi, Collider phenomenology of Hidden Valley mediators of spin 0 or $1 / 2$ with semivisible jets, arXiv:1712.07160 [INSPIRE].

[174] CMS collaboration, Search for new phenomena in final states with two opposite-charge, same-flavor leptons, jets and missing transverse momentum in pp collisions at $\sqrt{s}=13 \mathrm{TeV}$, JHEP 03 (2018) 076 [arXiv:1709.08908] [InSPIRE].

[175] M. Drees, H. Dreiner, D. Schmeier, J. Tattersall and J.S. Kim, CheckMATE: Confronting your Favourite New Physics Model with LHC Data, Comput. Phys. Commun. 187 (2015) 227 [arXiv: 1312.2591] [INSPIRE].

[176] D. Dercks, N. Desai, J.S. Kim, K. Rolbiecki, J. Tattersall and T. Weber, CheckMATE 2: From the model to the limit, Comput. Phys. Commun. 221 (2017) 383 [arXiv:1611.09856] [INSPIRE].

[177] J. Alwall, M. Herquet, F. Maltoni, O. Mattelaer and T. Stelzer, MadGraph 5: Going Beyond, JHEP 06 (2011) 128 [arXiv:1106.0522] [INSPIRE].

[178] J. Alwall et al., The automated computation of tree-level and next-to-leading order differential cross sections and their matching to parton shower simulations, JHEP $\mathbf{0 7}$ (2014) 079 [arXiv: 1405.0301] [InSPIRE].

[179] T. Sjöstrand, S. Mrenna and P.Z. Skands, PYTHIA 6.4 Physics and Manual, JHEP 05 (2006) 026 [hep-ph/0603175] [inSPIRE].

[180] T. Sjöstrand, S. Mrenna and P.Z. Skands, A Brief Introduction to PYTHIA 8.1, Comput. Phys. Commun. 178 (2008) 852 [arXiv:0710.3820] [INSPIRE].

[181] T. Sjöstrand et al., An Introduction to PYTHIA 8.2, Comput. Phys. Commun. 191 (2015) 159 [arXiv: 1410.3012] [INSPIRE].

[182] DELPHES 3 collaboration, J. de Favereau et al., DELPHES 3, A modular framework for fast simulation of a generic collider experiment, JHEP 02 (2014) 057 [arXiv:1307.6346] [INSPIRE].

[183] M. Selvaggi, DELPHES 3: A modular framework for fast-simulation of generic collider experiments, J. Phys. Conf. Ser. 523 (2014) 012033 [inSPIRE].

[184] A. Mertens, New features in Delphes 3, J. Phys. Conf. Ser. 608 (2015) 012045 [inSPIRE].

[185] J. Pumplin, D.R. Stump, J. Huston, H.L. Lai, P.M. Nadolsky and W.K. Tung, New generation of parton distributions with uncertainties from global QCD analysis, JHEP $\mathbf{0 7}$ (2002) 012 [hep-ph/0201195] [INSPIRE]. 
[186] M. Cacciari, G.P. Salam and G. Soyez, FastJet User Manual, Eur. Phys. J. C 72 (2012) 1896 [arXiv: 1111.6097] [INSPIRE].

[187] M. Cacciari, G.P. Salam and G. Soyez, The anti-kt jet clustering algorithm, JHEP 04 (2008) 063 [arXiv: 0802.1189] [INSPIRE].

[188] E. Conte, B. Fuks and G. Serret, MadAnalysis 5, A User-Friendly Framework for Collider Phenomenology, Comput. Phys. Commun. 184 (2013) 222 [arXiv:1206.1599] [InSPIRE].

[189] E. Conte, B. Dumont, B. Fuks and C. Wymant, Designing and recasting LHC analyses with MadAnalysis 5, Eur. Phys. J. C 74 (2014) 3103 [arXiv: 1405.3982] [InSPIRE].

[190] B. Dumont et al., Toward a public analysis database for LHC new physics searches using MADANALYSIS 5, Eur. Phys. J. C 75 (2015) 56 [arXiv:1407.3278] [INSPIRE].

[191] M. Grazzini, S. Kallweit, D. Rathlev and M. Wiesemann, $W^{ \pm} Z$ production at hadron colliders in NNLO QCD, Phys. Lett. B 761 (2016) 179 [arXiv:1604.08576] [INSPIRE].

[192] F. Cascioli et al., ZZ production at hadron colliders in NNLO QCD, Phys. Lett. B 735 (2014) 311 [arXiv:1405.2219] [InSPIRE].

[193] A. Giammanco and R. Schwienhorst, Single top-quark production at the Tevatron and the $L H C$, arXiv:1710.10699 [INSPIRE].

[194] M. Czakon, P. Fiedler and A. Mitov, Total Top-Quark Pair-Production Cross Section at Hadron Colliders Through $O\left(\alpha \frac{4}{S}\right)$, Phys. Rev. Lett. 110 (2013) 252004 [arXiv:1303.6254] [INSPIRE].

[195] M. Czakon and A. Mitov, Top++: A Program for the Calculation of the Top-Pair Cross-Section at Hadron Colliders, Comput. Phys. Commun. 185 (2014) 2930 [arXiv:1112.5675] [INSPIRE].

[196] N. Kidonakis, Soft-gluon corrections for $t W$ production at $N^{3} L O$, Phys. Rev. D 96 (2017) 034014 [arXiv: 1612.06426] [INSPIRE].

[197] H.-C. Cheng and Z. Han, Minimal Kinematic Constraints and m(T2), JHEP 12 (2008) 063 [arXiv:0810.5178] [INSPIRE].

[198] CMS collaboration, Performance of reconstruction and identification of tau leptons in their decays to hadrons and tau neutrino in LHC Run-2, CMS-PAS-TAU-16-002 (2016).

[199] ATLAS collaboration, Search for the direct production of charginos and neutralinos in final states with tau leptons in $\sqrt{s}=13 \mathrm{TeV}$ pp collisions with the ATLAS detector, Eur. Phys. J. C 78 (2018) 154 [arXiv:1708.07875] [InSPIRE]. 